\title{
Recognition of 3D Compressed Images and Its Traffic Monitoring Applications
}

by

Nicole Sharlene Love

Submitted to the Department of Electrical Engineering and Computer

Science

in partial fulfillment of the requirements for the degree of

Doctor of Philosophy

at the

\section{MASSACHUSETTS INSTITUTE OF TECHNOLOGY}

June 2004

(C) Nicole Sharlene Love, MMIV. All rights reserved.

The author hereby grants to MIT permission to reproduce and distribute publicly paper and electronic copies of this thesis document

in whole or in part.

Author... (

Depártment of Electrical Engineering and Computer Science April 14, 2004

Certified by

Berthold K. P. Horn Thesis Supervisor

Certified by $\ldots \ldots \ldots \ldots \ldots \ldots \ldots \ldots \ldots \ldots \ldots$

Ichiro Masaki Thesis Supervisor

Accepted by ..

Arthur C.Smith

Chairman, Department Committee on Graduate Students 


\title{
Recognition of 3D Compressed Images and Its Traffic
}

\author{
Monitoring Applications
}

by

\author{
Nicole Sharlene Love
}

\begin{abstract}
Submitted to the Department of Electrical Engineering and Computer Science on March 31, 2004, in partial fulfillment of the requirements for the degree of Doctor of Philosophy
\end{abstract}

\begin{abstract}
The need for improved security requires the development of better monitoring systems. These systems must manage large numbers of images sent over communication networks. For example, a high transmission load results when security cameras continuously transmit images. Operators are bored and fatigued from viewing cycled images on a few monitors.

In this thesis, we present a smart camera network that reduces transmission load and provides operators with relevant images. A smart camera is capable of compressing and processing images. Our 3D compression method (based on Mizuki's 2D method) retains features (contour, color, depth, and motion) that form the basis of object detection. The compressed image is used directly in segmentation.

To reduce transmission loads, we use mobile agents to filter the network. Mobile agents are dispatched to smart cameras with user defined goals that conditionally allow image transmission. For traffic monitoring, smart cameras determine traffic flow and accidents. Mobile agents send images or information based on image content.

The contribution of this work is the 3D compression method designed for processing compressed image data and the design of a system to improve camera centered networks using smart cameras and mobile agents. Our system can be used in security systems, where image content can promote an efficient transmission of images.
\end{abstract}

Thesis Supervisor: Berthold K. P. Horn

Title: Professor of Electrical Engineering and Computer Science

Thesis Supervisor: Ichiro Masaki

Title: Principal Research Associate of the Microsystems Technology Laboratories 


\section{Dedication}

I dedicate this thesis to my mother and my family:

My mother has shown me the value and strength of family. Her love and faith has guided me and continues to drive me to reach higher. She has always encouraged me to do my best.

I appreciate the love, encouragement, and support shown to me by my sisters (Kim Love, Renee Love, and Joya Love) and brothers (Frederick Love, Lance Love, Shayne Love and Keir Love). It is with their help that I continue to persevere. My nieces (Qaasima Love, Zakeea Love, Shana Johnson, Hassanah Lloyd, Salimah Lloyd, Tye-Tanisha Edwards, Tashema Edwards, Keirah Love, Keiretta Love, and Keiravona Love) and nephews (Mustaffa Love, Farrakhan Love, Shayne Love, Tremayne Phillips, Ibn Lloyd, Mustaffa Love Jr., Quasin Love, Keir Love, and Amir Williams) are stars that brighten my day whenever I see or hear from them. My grandmother is supportive of everything that I do; she is a joy to talk to, learn from, and love. My aunts (Sharon Caines and Laverne Thornton), uncles (Derrick Caines and Ronald Caines), and cousins (Kittha Caines, Sheres Caines-McKenzie, Passionne Thornton, Jose Thornton, Chera Caines, Claire Johnson, Megan Johnson, and Brooke Johnson) have always given me love, and the extra boost to achieve any objective.

My extended family, Kay Stancil and Atiya Stancil, with love, have emphasized the importance of physical and mental health, helping me to find balance between work and self. And Kimani Stancil, my best friend, has taught me to look inside myself to find the strength I need to complete my goals. 


\section{Acknowledgments}

A doctoral thesis requires the support and help of many people to produce. I would like to acknowledge and thank a few of the people who have helped me with this phase of my life and the production of this thesis. First, thank you to Dr. Kimani Stancil, who has been a constant supporter, encourager, and advisor since we met. His love and support have helped me through the good and bad times.

Dr. Ichiro Masaki and Dr. Berthold Klaus Paul Horn have supported and guided me through this research. Dr. Masaki has been a good mentor to me. His interest in and support of me and my development have assisted me greatly throughout this process. Dr. Horn has provided invaluable research advice and insight into camera calibration that is inherent in computer vision. Both my thesis supervisors have helped me to develop as a researcher and scientist.

I greatly appreciate my thesis readers, Dr. V. Michael Bove and Dr. Leonard McMillan, for their input and advice. I would like to thank Ron Wiken for the overall support and help building the system. A very special thank you to Dean Isaac M. Colbert for making life at MIT better, by providing shoulders to lean on. Marilyn Pierce, Margaret Flaherty, Debroah Hodges-Pabon, Carol Frederick, Dean Roy Charles and Cynthia Thompson have always provided a friendly face, kind words, and help with administrative issues. Dean Blanche Staton, Gail-Lenore Staton, and Lynn Roberson have provided me with emotional support and guidance thus enabling the completion of this degree.

Thank you to Kimani Stancil, Eric Brittain, Alison Morgan, Robbin Chapman, 
Aisha Walcott, Yajun Fang, Tamara Williams have provided support, attentive ears, advice, guidance, and friendship. I would like to thank Kimani Stancil for spending many hours discussing research ideas, reading drafts, and help with setting up the system. I appreciate the support from Eric Brittain, never hesitating to help when needed, always available to listen, give advice, and offer assistance. I would like to thank Kimani Stancil, Aisha Walcott, and Robbin Chapman for profreading portions of the thesis. Thank you to Yajun Fang for the many discussions of research topics in our area and for being an excellent office mate, group mate and friend.

Thanks to Mr. Tehani K. Finch, Dr, Sandra Brown, Dr. Marta Dark, Dr. Grum Teklemariam, Ms. Lisa Dyson, Dr. Manyalibo Matthews, and Mr. Jermane Massey for their friendship. The BGSA and ACME have been a great support to me. Friendships with Dr. John-Paul Clark, Mr. James McLurkin, Mr. Russell Eric Caulfield, Ms. Kimberly Waters, Mr. Lincoln Chandler, Mr. Reginald Bryant, Mr. Mark Hampton, Ms. Ishara Mills, Dr. Deirdre Lawrence, Dr. Lawrence Sass, Dr. Donald Brunson, and many others have brightened my days and provided advice and insight into graduate life at MIT. I appreciate library study sessions with Aisha Walcott and Eric Brittain that helped me to accomplish my writing and research goals. Thank you to Alicia Boozer for her friendship, support, and cheerful spirit.

My family and friends have always been both loving and supportive. Since we were undergraduates at MIT, Ms. Giovanna Gambrell and Dr. Valarie Thomas have uplifted my spirits, shared in triumphs and tragedies, and provided never waivering support. I appreciate the support shown to me by Vonnie Gambrell welcoming me into her family. Thank you to Odell Wilson, Delia Wilson, and the Maarifa Elementary 
and Middle School for their support. I would also like to thank Mrs Nelms, Ms. Rose Love, Mr. Bennett, Mr. and Mrs. Hoover, Ms. Randolph, the teachers, teacheraides, staff, and students of Harriet Tubman School (Newark, NJ), Camden Middle School (Newark, NJ), and St. Paul's School (Concord, NH) for providing me with an excellent foundation. 


\section{Contents}

$\begin{array}{lll}1 & \text { Introduction } & 16\end{array}$

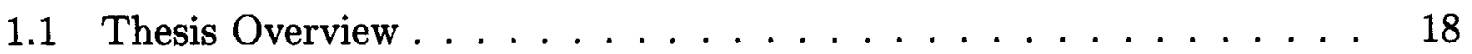

2 Intelligent Transportation Systems 20

2.1 Technologies and Systems $\ldots \ldots \ldots \ldots \ldots \ldots \ldots$

2.1.1 Transportation Management . . . . . . . . . . . 25

2.1 .2 Public Transportation $\ldots \ldots \ldots \ldots \ldots \ldots$

2.1 .3 Commercial Vehicle Operations . . . . . . . . . . . . 28

2.1.4 Traveler Information $\ldots \ldots \ldots \ldots \ldots$

2.1.5 Vehicle Control and Safety . . . . . . . . . . . 30

2.1 .6 Electronic Payment . . . . . . . . . . . . . . . . 31

3 System Overview 32

3.1 Network Topology $\ldots \ldots \ldots \ldots$

3.2 Image Sensors $\ldots \ldots \ldots \ldots \ldots \ldots \ldots \ldots \ldots \ldots$

3.3 Mobile Agents . . . . . . . . . . . . . . . . . . . . 37

3.4 Users . . . . . . . . . . . . . . . . . . . 38 
3.5 Alternative Network Topology . . . . . . . . . . . . . . . . . . . 39

4 Compression $\quad 41$

4.1 Image Compression Method . . . . . . . . . . . . . . . . . 44

$4.1 .1 \quad$ Depth Map . . . . . . . . . . . . . . . . 46

4.1 .2 Contour Extraction . . . . . . . . . . . . . . 46

4.1 .3 Contour Coding . . . . . . . . . . . . . . . . 47

4.1.4 Distance Extraction . . . . . . . . . . . . . . . . 57

4.1 .5 Color Extraction $\ldots \ldots \ldots \ldots \ldots \ldots$

$4.1 .6 \quad$ Mean Blocks . . . . . . . . . . . . . . . . . . . . . . 59

4.1.7 Motion Extraction $\ldots \ldots \ldots \ldots 60$

4.2 Image Decompression . . . . . . . . . . . . . . . . . . . 60

4.2 .1 Contour Decoder $\ldots \ldots \ldots \ldots$. . . . . . . . . . 64

4.2 .2 Color Decoding $\ldots \ldots \ldots \ldots \ldots$

4.2.3 Linear Interpolation $\ldots \ldots \ldots \ldots \ldots$

4.3 Compression Results $\ldots \ldots \ldots \ldots \ldots$. . . . . . . . . . . . . 67

4.3.1 JPEG Comparison $\ldots \ldots \ldots \ldots \ldots \ldots$. . . . . . 67

4.3.2 Bit-Rate Distortion . . . . . . . . . . . . . . . . 71

4.3.3 DCC vs. $\mathrm{MDCC} \ldots \ldots \ldots \ldots \ldots \ldots \ldots \ldots$

$\begin{array}{lll}5 & \text { Stereo Vision } & 79\end{array}$

5.1 Calibration $\ldots \ldots \ldots \ldots \ldots \ldots \ldots \ldots \ldots$

5.1 .1 Camera Model . . . . . . . . . . . . . . . 81

5.1 .2 Epipolar Constraint . . . . . . . . . . . . . . . . . . 82 
5.1.3 Relative Orientation ............... 83

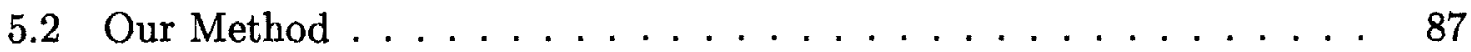

5.2 .1 Preprocessing .................... 88

5.2 .2 Depth ............................ 92

6 Vehicle Detection and Tracking $\quad 96$

6.1 Vehicle Detection . . . . . . . . . . . . . . 97

6.1 .1 Feature Selection . . . . . . . . . . . . 97

$6.1 .2 k$-Means Clustering . . . . . . . . . . . . . 98

6.1 .3 Dunn's Validity Index . . . . . . . . . . . . . . 100

6.1 .4 Detection Results . . . . . . . . . . . . . . . 102

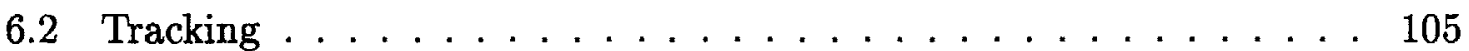

6.2.1 Matching Criteria . . . . . . . . . . . . 106

7 Software Agents $\quad 109$

7.1 Implementation . . . . . . . . . . . . . . . . . . . . 111

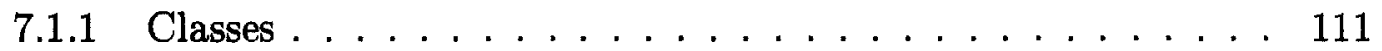

7.2 Enhancements . . . . . . . . . . . . . . 117

7.2 .1 Image Management . . . . . . . . . . . . . . . . 117

7.2 .2 Route Guidance . . . . . . . . . . . . . . . . . . . . 118

8 Additional Applications $\quad 119$

8.1 Vehicle Detection by Color . . . . . . . . . . . . . . . . 119

8.2 Lane Detection . . . . . . . . . . . . . . . . . . 120 
9 Conclusion

9.1 Future Research . . . . . . . . . . . . . . . . . . . 127 


\section{List of Figures}

3-1 Network Topology . . . . . . . . . . . . . . . . . . . . . 34

$3-2 \quad$ PC Implementation $\ldots \ldots \ldots \ldots \ldots \ldots \ldots \ldots$

3-3 Centralized Network Topology . . . . . . . . . . . . . . . . . . 40

$4-1$ Representation of Data . . . . . . . . . . . . . . . . . 42

$4-2$ Compression Algorithm . . . . . . . . . . . . . 45

$4-3 \quad$ Thinning Mask $\ldots \ldots \ldots \ldots \ldots \ldots \ldots$

4 4-4 DCC Contour Coding Flow Chart . . . . . . . . . . . . . 48

4 4-5 Direction Codes . . . . . . . . . . . . . . . . . . . 49

$4-6$ Branching Configurations $\ldots \ldots \ldots \ldots$

4-7 MDCC Contour Coding Flow Chart . . . . . . . . . . . . 50

4-8 Example Contour . . . . . . . . . . . . . . . . . . 51

$4-9$ Bits to encode a contour $\ldots \ldots \ldots \ldots \ldots$

$4-10$ Color Extraction $\ldots \ldots \ldots \ldots \ldots \ldots \ldots \ldots$

$4-11$ Example of Color Extraction . . . . . . . . . . . . . . . . 58

$4-12$ Mean Blocks . . . . . . . . . . . . . . . . . . . 59

4-13 Mean Blocks Example . . . . . . . . . . . . . . . . . . 59 
4-14 Original, Compressed and Error Images . . . . . . . . . . . . . . . 63

4-15 Decoder Flowchart . . . . . . . . . . . . . . . . . 64

4-16 Decompressed image before and after smoothing. . . . . . . . . . . . 67

4-17 JPEG Compression Comparison . . . . . . . . . . . . . . . 68

4-18 JPEG Compression Comparison . . . . . . . . . . . . . . . . 69

4-19 Comparison of Edge Images . . . . . . . . . . . . . . . . . 70

4-20 Bit-rate distortion curve for "lena" and "parrots" images . . . . . . . 71

4-21 Sample color and edge images from each set . . . . . . . . . . . 72

4-22 Compression Ratio of Edge Images with maximum number of direction codes equal to $64 \ldots \ldots \ldots \ldots$. . . . . . . . . . . . . . . 73

4-23 Sample of Lighting Shift . . . . . . . . . . . . . . . . . 74

4-24 Range of Percentage Increase in Compression Ratio of MDCC over DCC for edge images with maximum number of direction codes 16,32 ,

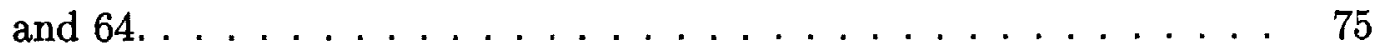

4-25 Compression Ratio of Images with maximum direction codes of 64 . . 77

4-26 Range of Percentage Increase in Compression Ratio of MDCC over DCC for full color images with maximum number of direction codes 16,32 , and $64 \ldots \ldots \ldots \ldots \ldots \ldots$. . . . . . . . . . . . . . . 77

$5-1$ Pinhole Camera Model . . . . . . . . . . . . . . . . . . 82

5-2 Epipolar Constraint ..................... 83

5-3 Relative Orientation . . . . . . . . . . . . . . 84

5-4 Manual Correspondence ................. 86 
5-5 Average error per point pair from corresponding point to epipolar line 88

5-6 Stereo Vision Flow chart . . . . . . . . . . . . . . . . . 89

$5-7$ Sobel Kernel . . . . . . . . . . . . . . . . . 90

5-8 Original image, positive and negative vertical edges . . . . . . . 90

5-9 Additional Vertical Edges Example . . . . . . . . . . . . . 91

5-10 Subpixel Approximation ...................... 92

5-11 Correspondence using epipolar constraint and normalized correlation. 94

5-12 Sample of correspondences and depths . . . . . . . . . . . 95

$6-1$ Histogram of Depth . . . . . . . . . . . . . . . 98

6-2 Scatter Plot of Motion Vectors vs. Depth . . . . . . . . . . . . . 99

6-3 Example of actual, observed, and detected objects . . . . . . . . . 103

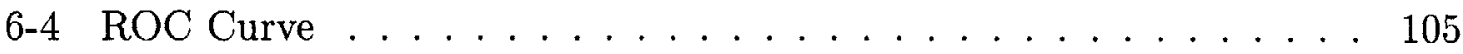

6-5 Tracking Algorithm . . . . . . . . . . . . . . . 106

7-1 Aglet Environment . . . . . . . . . . . . . . 110

7-2 Interactive Window at the control center (Photo from KYTC / TRW / MELCO / TRIMARC) . . . . . . . . . . . . 115

7-3 Interaction between control center and image sensor . . . . . . . . 116

8-1 Detect yellow vehicle based on color . . . . . . . . . . . . 120

8-2 Lane Detection . . . . . . . . . . . . . . . . 121

8-3 Histogram with thresholding . . . . . . . . . . . . . . . 122

8-4 Original image with depth map . . . . . . . . . . . . . 123 
8-5 Depth histogram with thresholding . . . . . . . . . . 124

8-6 Vehicles eliminated from scene . . . . . . . . . . . . . . . . 124

8-7 Lane Detected . . . . . . . . . . . . . . . . . . 124 


\section{List of Tables}

3.1 Sony XC-711 specifications . . . . . . . . . . . . . . . . 36

4.1 Number of bits required for codes in DCC and MDCC $\ldots \ldots \ldots 2$

4.2 Number of bits to encode example in DCC . . . . . . . . . . 52

4.3 Number of bits to encode example in MDCC . . . . . . . . . . . 54 


\section{Chapter 1}

\section{Introduction}

In video surveillance systems visual information is used to monitor surroundings, identify suspects, witness illegal activity, and determine the cause of accidents. Generally, for systems relying on video information, image sensors (cameras) are strategically placed to monitor specific locations. Since an important goal of these systems is to maintain safety and security using image sensors, we consider how image information is stored, transmitted, processed and retrieved. Images contain a large amount of data, thus it is a challenge to determine an efficient way of transmitting and processing them. In this thesis, we address this challenge in the context of traffic monitoring.

The goal of our research is to reduce the transmission load while maintaining the quality of available traffic information. We present two methods to reduce the transmission load and to efficiently manage the transmission of images. Our methods to reduce the transmission load involve 1) reducing the size of images sent over the network using a 3D compression method which retains contour, color, depth and motion information, and 2) reducing the number of images sent over the network. 
Note, the methods we present can be implemented in any system that continually transmits images, but we focus our attention on a traffic monitoring system.

Our compression method is designed to enable processing on the compressed image data. We modify Mizuki's contour based compression method by changing the motion vectors to be contour based, adding depth information to enhance processing of the compressed image data, and finally, modifying the contour coding to increase compression and improve contour representation.

A contour based compression method produces compressed image data that is conducive to further processing. The attributes (contour, color, depth, and motion) used in our compression method form the basis of our processing algorithms. We demonstrate the effectiveness of our compression method by detecting and tracking objects. Many image processing algorithms use edge, motion, and/or depth information as an initial step. If an image is to be processed after transmission, retaining accurate edge information is essential. In contrast, JPEG produces data that needs to be decompressed before further processing. The decompressed image is blocky, altering edge information. The JPEG image does not contain depth and motion information, making further processing of the image inaccurate. Our compression method ensures edge, motion and depth information are available for further processing and processes the compressed image data without prior decompression.

Additionally, we use mobile agents to filter the network. A mobile agent is an autonomous program which can travel from one computer to another in order to achieve preset goals. A mobile agent can monitor sensor information and send traffic information or traffic images over the network based on the operator's goals. If 
directed, the agent may travel to other computers gathering information to be relayed to the operator.

Our system is designed primarily for a distributed network, where each image sensor processes images locally. The mobile agent sends the compressed image based on the contents of the image. The processing of the image determines its contents. In traffic monitoring, vehicle detection and tracking are essential to calculating traffic information from the images. We detect and track vehicles by processing the 3D compressed image data. Furthermore, we remind the reader that the components of our system can be used in any system involving image transmission, specifically when the image or a description of the contents of an image can be sent. Consider for instance, a system custom designed for hospital intensive care units (ICU) to check the health and safety of patients. Images can be useful, but simply knowing that patients are positioned correctly in bed and the output of monitoring devices may be sufficient for monitoring.

\subsection{Thesis Overview}

In this thesis, we present our system as it relates to traffic monitoring. We discuss the research goals and status of ITS (Chapter 2), since traffic monitoring is a key component of Intelligent Transportation Systems (ITS). To present our system, we detail the different hardware devices and software programs used in the implementation (Chapter 3). As the stated goal is to reduce transmission loads, we begin the discussion by explaining the 3D compression method and results (Chapter 4). Since 
the 3D compression method necessarily requires us to collect or compute distance information using stereo vision, we include the stereo vision calculations and results (Chapter 5). To use mobile agents to manage the transmission of images and/or information, image content must be extracted (i.e. vehicle detection and tracking information), we obtain this information using $k$-means clustering to group features (Chapter 6). A basic discussion of mobile agents and how they work is also included (Chapter 7). Ultimately, we process 3D compressed image data and we show the significance of this processing with preliminary results using other applications (Chapter 8). We conclude by discussing key features of the system, our observations, and future research directions. 


\section{Chapter 2}

\section{Intelligent Transportation Systems}

Traffic monitoring is a key component of Intelligent Transportation Systems (ITS). Monitoring provides information about road and traffic conditions. The goal of ITS is to improve the safety, security, overall efficiency, and convenience of transportation with regard to people and goods. We apply our system to Intelligent Transportation Systems to demonstrate feasibility. This chapter discusses the history, features and potential of ITS for surface transportation.

The first advanced electronic systems for transportation were developed in the 1960's. Problems with congestion, accidents, and pollution were a large concern and these problems were estimated to increase significantly with increases in population. One-fourth of Los Angeles' land was devoted to roadways at this time [26]. It was clear that more roads would not solve current and future transportation problems. Instead, more efficient use of existing roads provided the most promise for solving this problem. With this in mind several systems such as, ERGS (Electronic Route Guidance System), Automatic Vehicle Identification and Location, Computer-Controlled 
Traffic Systems and Merging Control Systems were developed and tested.

Some of the systems designed during the 1960's are presented in a Special Issue of the IEEE Transactions on Vehicular Technology [73]. These systems used mobile radio communication, inductive loop detectors, and other technologies to enable road to vehicle communication. With the establishment of communication between moving vehicles and roadside devices, systems were being developed to improve surface transportation.

ERGS, developed by the Bureau of Public Roads (now the Federal Highway Administration, FHWA), used roadside devices to communicate with vehicles through inductive loops and to communicate with a central computer through a hardwired connection [66]. Using an in-vehicle device, the user would enter a destination code which would be transmitted to a roadside device. The roadside device communicates with the central computer (where current traffic data is located) and immediately transmits instructions to an in-vehicle display [46]. ERGS was scrapped in 1971, when the congressional appropriations committee did not approve the budget request [66].

Without government funding, research for these systems did not continue. It was not until twenty years later, in the 1980 's, with the near completion of interstates, that developing electronic systems to improve transportation resurfaced in the United States. The California Department of Transportation (Caltrans) concluded from it's study for future road construction programs that no amount of construction could solve their current congestion problems [48]. With projected growth in congestion, construction was not the solution. In October 1986, Caltrans held a conference to discuss the use of technology to solve the congestion problem [66]. From this conference, 
a group of experts worked to develop a national agenda for surface transportation research. An organization sponsored by FHWA was formed in 1988, consisting of government, industry and university researchers, called Mobility 2000 [66]. "Mobility 2000 Presents Intelligent Vehicle Highway Systems" was written in 1990 recording the organizations' discussions in workshops and conferences. This document was presented at the National Leadership Conference held by the Secretary of Transportation in May of 1990. Following this conference, ISTEA (Intermodal Surface Transportation Efficiency Act) was passed in 1991 by then President George Bush, and provided funding for a national Intelligent Vehicle Highway System (IVHS) program and the creation of IVHS America (now ITS America).

ISTEA provided $\$ 660$ million for an IVHS program for a 6-year period. Included in this program was funding for the planning, feasibility, and operational tests of IVHS technologies. Funding was also designated for the development of a completely automated highway and vehicle system by 1997 [68]. This legislation supported the use of technology for transportation systems. In 1998, President Bill Clinton passed TEA-21 (Transportation Equity Act for the 21st Century). This law provided $\$ 1.282$ billion for FYs 1998-2003 to fund the Intelligent Transportation Systems (ITS) program [72]. More than $50 \%$ of the funding is for the integration and deployment of ITS. The government has continued to support ITS as a means of improving surface transportation. A law to extend TEA-21 for 5 months was passed on September 30, 2003. Currently a bill, SAFETEA (Safe, Accountable, Flexible, Efficient Transportation Equity act of 2003) is before the senate which will fund ITS ( $\$ 726$ million) until FY 2009. SAFETEA provides similar funding as TEA-21 with less emphasis on 
deployment and more emphasis on safety and security.

Today, vehicle safety has been improved in a number of ways. Air bags, anti-lock brakes, adaptive cruise control, and other injury-reducing technology has improved vehicle operator safety. We expect to improve safety by increasing seat belt and child safety belt usage, collision warning systems, speed enforcement, rapid detection of accidents and dispatch of emergency response units (paramedics, police, tow trucks, etc.). To complement these advances, it is important to identify and maintain the quickest traffic routes to an accident through traffic monitoring, warning, and detour systems.

Besides safety, security has become extremely important in the United States over the past few years. The newly created Department of Homeland Security and the Transportation Security Administration are focused on the need for increased security in airports, water ports, roadways, and railways. Securing borders on land, water, and air has always been of concern and current methods are being assessed. The Department of Transportation is addressing vulnerabilities in subways, tunnels, and stations. Preventing major problems and improving response to security problems are goals of the recently established (2002) Department of Homeland Security (previously the Office of Homeland Security created September 20, 2001). Image Sensors have been added to tunnels for both traffic information and security. Traffic monitoring systems with image sensors will be essential for security at national borders, subways, tunnels, and stations. Security is dependent upon an efficient transportation system. Maintaining the movement of vehicles is an essential component.

Increasing efficiency (mobility and productivity) of surface transportation sys- 
tems, through ITS, requires reducing congestion on the roads. Since, the number of people who work in the city and live in the surrounding areas has increased significantly, the commute in the mornings and evenings are the main cause of congestion on roads. Congestion results in air pollution, accidents, and frustration amongst drivers. Commuter rails were added to reduce the number of drivers on the road by giving drivers another choice, public transportation. Presently, cities with fairly new commuter rail systems have failed to reduce congestion. By comparison, congestion has increased in these cities relative to cities without rail systems [12]. An Intelligent Transportation System which has been effective in reducing congestion is congestion pricing [67]. Drivers are charged more for driving during peak times (rush hour). California implemented congestion pricing in four toll lanes and travel time reduction is estimated at twenty to forty minutes. Rush hour traffic speeds were maintained at $65 \mathrm{mph}$ verses $35 \mathrm{mph}$ on non-toll lanes.

Congestion may also be reduced by providing travelers with information. Traffic information enables drivers to make informed decisions about travel routes, times of travel and modes of transportation. Several states' Department Of Transportation (DOT) have made current traffic information of highly traveled roads available to drivers through Highway Advisory Radio and Internet sites. The San Francisco 'TravInfo' internet site has significantly affected travelers behavior, such that $81 \%$ of travelers receiving specific route information changed their travel based on information provided by the site [49]. Using the internet some DOTs have also provided traffic images to drivers and these images are updated at intervals of 1-10 minutes depending on the system. There are a small number of DOTs which provide live traffic 
video for select locations. The convenience of providing up-to-the-second (current) information can only help to reduce congestion and increase the overall efficiency of transportation systems.

\subsection{Technologies and Systems}

ITS rely on new technologies to improve transportation systems. Research has been done in government, universities, and industry to find methods of improving transportation, either by systems developed for vehicles, monitoring, communication, or management. There are six main areas of interest in ITS, 1) Transportation Management, 2) Public Transportation, 3) Commercial Vehicle Operations, 4) Traveler Information, 5) Vehicle Control and Safety, and 6) Electronic Payment. In the remainder of this chapter we discuss each of these areas and if applicable, the relevance to traffic monitoring. For each area, we present the current status of the research and its implementation.

\subsubsection{Transportation Management}

A transportation management system requires efficient use of equipment, personnel and resources. Responding to emergencies, and maintaining, updating and modifying operations are all components of transportation management systems. Transportation Management Centers (TMC) have been created in $2 / 3$ of the largest 75 metropolitan areas in the United States of America [39]. Their focus is to monitor freeway traffic and provide early notification of incidents. Plans are being developed 
to utilize both new and old technologies.

The goal of the TMC is to obtain information and communicate with devices, drivers and emergency response units. The TMC in the state of Maryland has analyzed the most cost efficient way to implement its transportation telecommunication goals [52]. Maryland's TMC will use existing communication equipment, existing network topology, and a combination of new communication technologies to reduce costs. Several other states (e.g. Maine, Virginia, and Arizona) are also analyzing TMC goals and planning a cost effective architecture.

In order to implement ITS, data must be transmitted from the road sensors (sensors which are located along the road) to the TMC. In California, for instance, it is estimated that two gigabytes of data are transmitted per day [79]. Accessing this data by both the TMC and interested drivers requires communication systems with high bandwidth. Currently, systems use DSRC (Dedicated Short Range Communication) that send microwaves over short distances to transmit up to $54 \mathrm{Mbps}$ of data. The increase use of image sensors on the road has caused a move to fiber-optic networks that have a larger bandwidth (50-100 Mbps).

The technology to communicate with vehicles and drivers is also being developed and implemented. Automatic Vehicle Location (AVL) is a technology which uses GPS (Global Positioning System) and GIS (Global Information Systems - database of road information) to track and locate vehicles. TMC can use Computer-Aided Dispatch Systems to send response units to accident sites. Note, accidents involving vehicles with telematics (in-vehicle systems that use wireless communication and GPS tracking [83]) will receive quicker responses. The TMC can also provide travel routes 
to emergency response units based on traffic monitoring information.

PeMS (Performance Evaluation Monitoring System) software developed at UC Berkeley analyzes "traffic patterns and predicts travel times up to an hour in advance" [79]. PeMS allows traffic managers to quickly view trends in traffic to address potential problems and allows traffic planners to analyze new routes.

Traffic Monitoring systems are beneficial to Traffic Management Centers, drivers, and emergency responders. DSRC-microwave sensors allow operators to obtain traffic flow and average speed information. Inductance loop detectors (installed in the pavement) provide traffic flow and speed as well as vehicle length information for classification. Image Sensors have also been added to several highways, tunnels, and streets in the U.S. to obtain traffic information and verify road and weather conditions.

\subsubsection{Public Transportation}

The goal of public transportation is to provide a safe and reliable transport service to customers. Generally, public transportation was developed to reduce congestion and make cities accessible to the masses. It is believed that ridership will increase when travelers are provided with more information about the location of buses, travel time estimates and/or traffic conditions. Public transportation systems currently use AVL, GPS, and Communication Systems to provide this information. In Denver, use of an AVL system improved bus service and decreased late arrivals by appropriately routing buses in real-time [49]. These systems will also help to improve the dispatch 
of buses, provide quicker responses to vehicle failure and criminal activity. ComputerAided Dispatch Systems (CAD) and Mobile Data Terminals will be used to transfer connections and restore service in the case of emergencies. Additionally, public transportation systems have installed satellite-based CAD systems that provide more effective and safer service for the public. An AVL/CAD system, in Missouri, reduced the number of buses by up to $10 \%$ on some routes with no reduction in customer service [49]. GIS systems will also be used for route planning and bus scheduling. Public transportation systems also hope to increase service and customer satisfaction by implementing Traffic Signal Priority Systems, where buses at a traffic light will be given priority. Ten cities, using this system, have shown $2-20 \%$ improvement in bus travel time with a corresponding improvement in on-time performance [49].

\subsubsection{Commercial Vehicle Operations}

The movement of goods raises some concern for accidents, theft, and terrorism. By using AVL to track the location of vehicles and to match the route to an intended destination, the USDOT will increase safety and reduce the theft of commercial vehicles. Automatic Vehicle Identification Systems also help by providing commercial operator verification. CAD systems have increased the number of pickups and deliveries by $5-15 \%$ [49]. Trucks were involved in $3,560,956$ crashes (58\% of highway crashes) in 2001 [8]. These numbers are being reduced by providing commercial vehicles with technologies to improve visibility in blind spots with image sensors and collision warning systems. 
The Highway Act of 1956 involved the federal government in the creation of the interstate road system. Based on research done by the American Association of State Highway Officials (now American Association of State Highway and Transportation

Officials) that showed the effect of large vehicles on roadways, the Highway Act of 1956 added weight regulations [18]. States were required to enforce the requirements, weigh stations were already implemented by some states, while others had to fund new infrastructures. To reduce congestion at weigh stations and increase safety around a weigh station, weigh-in-motion systems were developed in 1956 . Weigh-in-motion systems allow trucks to be weighed while they travel highway speeds. There is currently concern with calibration and accuracy of these systems, but the potential to improve mobility and safety makes this research important for transportation efficiency.

\subsubsection{Traveler Information}

Pre-trip Information Systems (PTIS) help drivers choose the mode of transportation, route, and departure time. These systems can be divided into four types, 1) General Service Information, which gives information about construction areas, traffic, weather and road conditions, 2) Itinerary Planning determines travel routes from one location to another, 3) Real-time Information, which provides current traffic flow information on major roadways, and 4) Multimodal Traveler Information, which joins traffic, planes, trains, buses, and shuttle information to enable door to door travel. The location of buses, trains and/or shuttles can be disseminated using kiosks, the internet, telephones, PDAs and/or video monitors at bus stations, airports, and train 
stations. In Finland, 95\% of customers surveyed thought real-time transit information displays were helpful [49]. Traffic Information can be distributed using the same technology as well as changeable message signs and highway advisory radio. Currently, many states provide traveler information and traffic images on the internet. TrafficLand, Inc. has joined with DOTs to provide personalized traffic images for commuters at a cost of $\$ 3.95 /$ month [84]. The user specifies which roads are of interest and a personalized set of images are available when the driver logs on the website to make viewing traffic images easier and more convenient.

\subsubsection{Vehicle Control and Safety}

In-vehicle systems to improve safety have been designed and implemented. Obstacle detection and collision warning systems alert drivers to potential dangers. Night vision systems reduce accidents by increasing visibility of objects on the road.

Research is also being done on Cooperative Vehicle-Highway Automation Systems (CVHAS) to automate driving tasks through cooperation with an intelligent infrastructure [39]. These systems focus on platooning (vehicles automatically following a lead vehicle without driver control) through communication between vehicles on the road.

The automotive industry is looking to grow business by providing more features and more services to drivers. Currently a large number of new vehicles are equipped with CARWINGS, InterNavi, OnStar, or G-book systems which provide route-guidance, theft-protection, emergency dispatch services, and other needed ser- 
vices to drivers $[77,78]$. These services are implemented using a wireless phone to communicate with drivers and GPS (Global Positioning Systems) to provide the location of vehicles. Adaptive Cruise Control has also been added to four new automobile models to provide a warning based on the relative distance between a driver and the next car ahead [86]. Many commercial vehicles now have Collision Warning Systems which have significantly reduced the number of accidents [76]. Systems designed for vehicle application have focused on reducing accidents and increasing driver comfort.

\subsubsection{Electronic Payment}

Whether as a matter of convenience in the case of TrafficLand, Inc. or to minimize travel time as in the case of congestion pricing, continued use of technology to increase vehicle speed and efficiency of payment has also received much attention. Paying for transportation services electronically, is becoming more and more common. With EZPASS for toll collection and transit passes, these technologies are making electronic payment very convenient for drivers. There are future plans to use toll collection technology for parking and fast-food drive-thrus. New Jersey's E-ZPASS have reduced delays by $85 \%$ for all vehicles, including those vehicles that do not have an E-ZPASS saving an estimated $\$ 19$ million in delay costs and $\$ 1.5$ million in fuel cost each year

. In Europe, a smart card can be used for several city services (such as public transit, shops, libraries, and swimming pools) with user acceptance and satisfaction ranging from $71-87 \%[49]$. 


\section{Chapter 3}

\section{System Overview}

Our system is designed to reduce transmission loads of systems that continually send images from many image sensors. Currently, images from hundreds of image sensors are sent to a centralized location and cycled through for viewing on a few video screens. This type of system requires high bandwidth. To reduce transmission loads, we scale down the data that is transmitted by compressing the images, and we use mobile agents to decide when to send images. Our compression method is designed to retain key features important for further processing.

The system consists of four components: 1) the network, 2) image sensors, 3) mobile agents and 4) users. The network relies on the communication between computers and other devices in establishing a medium for sending/receiving data. The network topology determines the functionality of our system and depends on computers, devices and signal connections. A distributed network divides processing among the devices of the network. In a centralized network, information is processed at a single node. Centralized and distributed networks are both commonly used. In prac- 
tice, the choice of network topology depends on cost and system applications. In this chapter, we focus our discussion on a distributed network, and briefly, we discuss how our system would apply to a centralized network.

In a distributed network, cameras process the images. The addition of a processor at an image sensor is an added upfront cost. The processor in effect reduces the required bandwidth. In the case of leased lines (\$200-\$300/month for each Mbps), bandwidth is extremely costly. The cost of having a processor at each image sensor is an additional but acceptable expense that reduces the total cost, through the reduction in bandwidth. In this thesis, image sensors will generally refer to the combination of an image sensor and processor. Thus, we understand image sensors as devices that acquire, compress and process images, as well as host mobile agents.

The mobile agents are sent by system users to the image sensors. At the image sensor, the mobile agent monitors traffic information. In accordance with the users' goals, the mobile agent acts or responds based on information gathered. Upon completion of the user's goals, a mobile agent can be retracted (returned to the users' machine) or disposed (deleted from the network).

The mobile agents are controlled by the users of the system. The operators at the Traffic Management Centers (TMC) and travelers are the main users in a traffic monitoring system. Users send a mobile agent to the appropriate image sensor(s) with preset goals. These goals can be as simple as sending an image to the user. A more complex goal could require several mobile agents to work together. For example, a route guidance system may be implemented by sending several agents to various potential routes, where each agent gathers traffic information and determines 
collectively the best route to present to the user.

The network topology, image sensors, mobile agents, and users are the main components which form the basis of our system. In the sections that follow, we present detailed information about each component with examples of usage. Mobile agents are discussed in more detail (including their implementation) in Chapter 7.

\subsection{Network Topology}

As previously mentioned, our focus is on a distributed network. An example of the configuration is shown in Figure 3-1. The distributed network not only enables processing by the image sensors, but also allows direct access to image sensors by all users. We use mobile agents to manage the communication between the user and

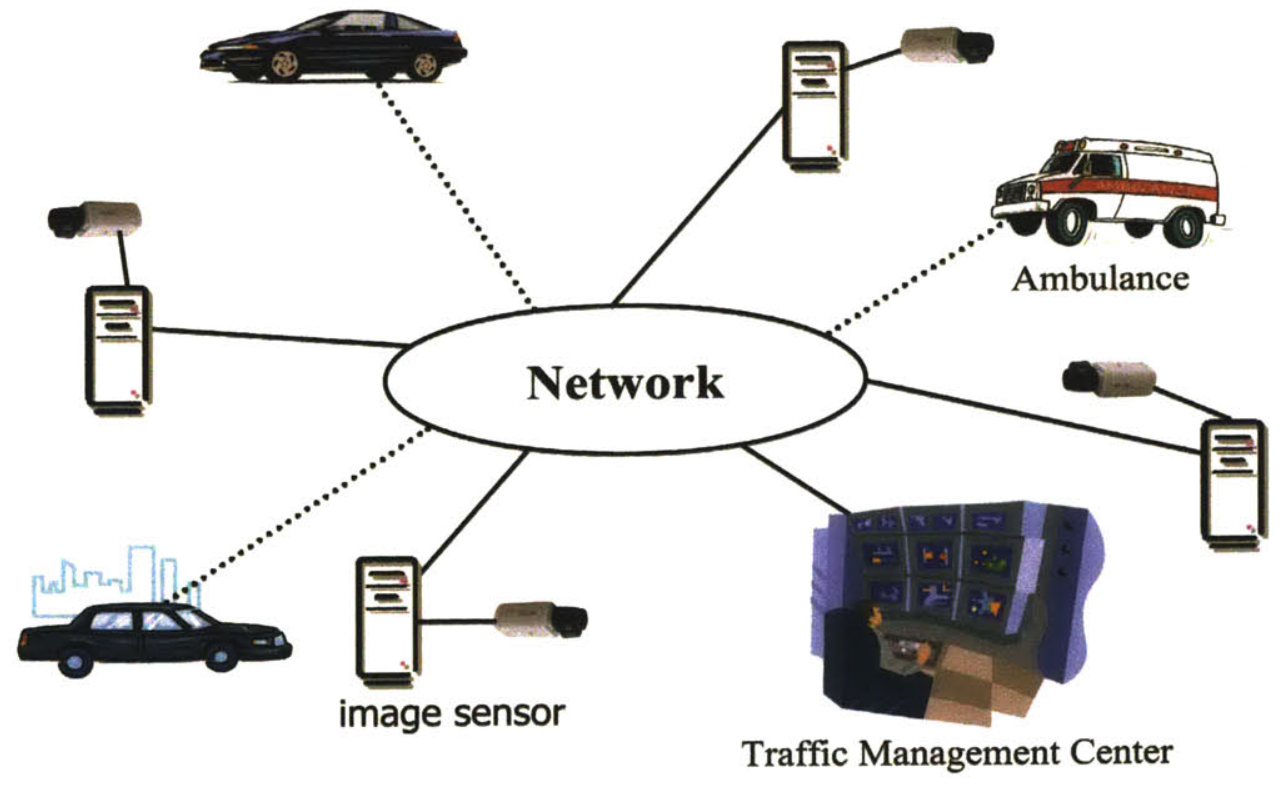

Figure 3-1: Network Topology

image sensors. The mobile agents determine if and when to transmit over the network, thereby reducing the transmission load of the network. In order to manage the 
network, the mobile agents are given preset goals that determine what to transmit and under what conditions. The images are processed at the image sensor producing traffic flow information, as well as preset labels (normal, congestion, heavy congestion).

\subsection{Image Sensors}

The image sensors in our system have several features. Besides acquisition, the image sensor must perform a 3D compression, process the images to determine content, and enable mobile agents to access images and information. Images are acquired using a three camera system shown in Figure 3-2. The three camera system acquires two monochrome and one color image simultaneously. In order to obtain all three images simultaneously, two frame grabbers (PCI Boards) are needed. One frame grabber (Matrox Corona-LC) is used to capture the color image and the other frame grabber (Matrox Meteor II-MC) is used to capture the two monochrome images. The three cameras are Sony XC-711 CCD cameras; the specifications for the cameras are provided in Table 3.1. The images are stored on the hard drive and all processing is performed on the CPU of the host computer (Dell Dimension $4550-2.5 \mathrm{GHz}$ ). The two frame grabbers are synchronized by providing a signal from one frame grabber to the other.

Once the images are acquired, the images are compressed using our 3D compression method, described in detail in Chapter 4. To generate the 3D information for compression, our system uses a stereo vision algorithm. Stereo vision uses two images 


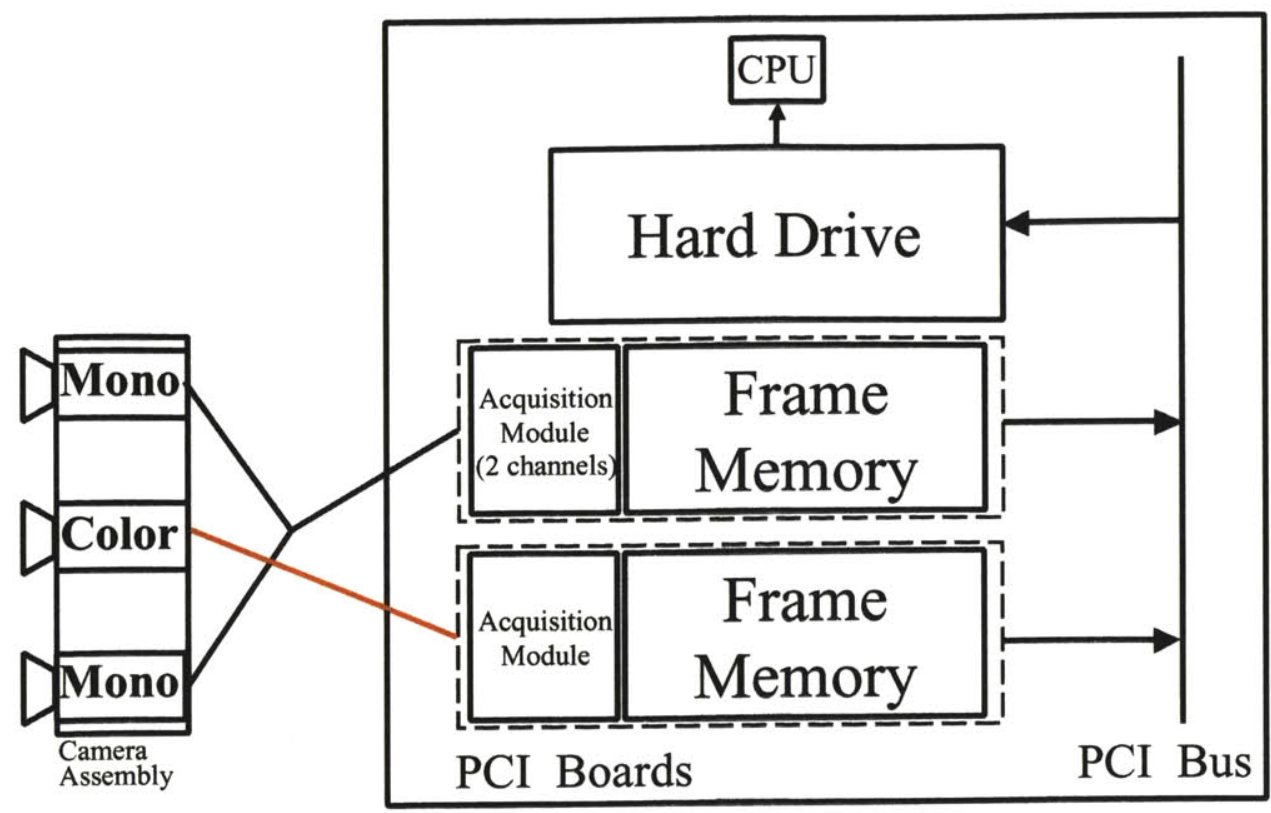

Figure 3-2: PC Implementation

\begin{tabular}{|c|c|}
\hline Pickup device & $2 / 3$-inch IT CCD \\
\hline Effective picture elements & $768 \times 493$ \\
\hline Cell size & $11.0 \times 13.0 \mu \mathrm{m}$ \\
\hline Sensing area & $8.8 \times 6.6 \mathrm{~mm}$ \\
\hline Output modes & $2: 1$ interlaced, noninterlaced \\
\hline
\end{tabular}

Table 3.1: Sony XC-711 specifications

to determine the distances of an objects' features from the cameras (the details of the algorithm are given in Chapter 5). The contents of the images are then determined by processing the 3D compressed images (discussed in Chapter 6). The image sensor hosts the mobile agent in a separate environment which limits the access of the mobile agent to the physical memory of the image sensor. With the traffic flow information and preset labels (normal, congestion, and heavy congestion) that describe current traffic conditions, a mobile agent can respond appropriately based on the preset goals. Mobile agents require read privileges (on the image sensors) of current compressed data and traffic information to enable transmission to system users. 
The environment dedicated to mobile agents in the image sensors can be set to give mobile agents from the TMC unlimited access and simultaneously restrict the access of other mobile agents to compressed data and specific traffic flow information.

\subsection{Mobile Agents}

The criteria of the mobile agents can be set to send images continuously or only when there is congestion or an incident. Mobile agents may also be directed to obtain traffic flow information, such as average speed or traffic volume. The actions of a mobile agent are determined by the user's goals (that are programmed prior to dispatch of the agent). We implemented simple goals, but the mobile agents can be designed to accomplish more complicated goals, that may require communication between mobile agents and involve retrieving information from several image sensors.

Mobile agents can be used in route guidance systems to determine the best route. They can find information on potential paths and sort the paths based on estimated time of arrival or distance. A stationary agent (located on the user's computer) can maintain the user's vehicle state information (speed, current location and heading) and communicate with the mobile agent(s). A mobile agent can send driving directions to the user.

Mobile agents also need to be disposed (permanently removed from the network) to efficiently use memory and processor resources on image sensors. Mobile agents can be removed either at a preset time, directly by the user or stationary agent, or upon completion of a user's goals. Determining the best method to dispose of the 
mobile agent depends on the application. In Chapter 7, we discuss implementation of simple goals for mobile agents.

\subsection{Users}

With our system, users can benefit from using a distributed image sensor network, by directly accessing the desired image sensor. Image sensors will process images and determine their content. Mobile agents can transmit compressed image data or information. In a distributed network, requests for images and information will not be directed to the TMC, therefore, users can obtain information directly from image sensors, thus taking out the 'middle man.' The TMC can then focus more fully on planning, monitoring, analyzing, and responding to changing traffic conditions. The network bandwidth at the TMC will no longer be devoted in large part to the transmission of images and information to users.

Along with mobile agents, direct control of image sensors must be restricted to the TMC for security purposes. Controlling the image sensors requires remote access. In the case of an accident captured by an image sensor, the TMC may elect to give only emergency units access to images and alternatively only provide travelers access to traffic information (notification of an accident and current traffic flow). Limiting the access of the image sensor will provide up to date images for emergency personnel and control what the travelers view.

The primary goal of the TMC (Traffic Management Centers) is to collect traffic information for immediate response, planning, analysis, and evaluation. Normally, 
live traffic images are viewed by operators and traffic counts, volume, and average speed are collected by on-road devices. Images can be used in conjunction with or in lieu of on-road devices. In many DOTs, traffic images are also used for verification of weather, road, and traffic conditions. Over the past ten years, research has been applied to collecting traffic information and detecting incidents using images. The accuracy of these systems to obtain traffic information has not yet surpassed onroad devices, but the potential does exist for future improvements. Image sensors are very convenient since they are non-intrusive devices, and provide operators with visual confirmation and incident detection. The TMCs are looking forward to using image sensors for traffic monitoring. Images of an accident enable rapid response and help to determine the equipment and personnel needed to respond to the accident. Our system can be used to monitor and collect traffic information for planning and analysis, and to enable rapid response to an incident that is detected by image sensor.

\subsection{Alternative Network Topology}

Although DOT Intelligent Transportation Systems are moving toward distributed networks, many still have a centralized network topology as in Figure 3-3. A centralized network requires all image sensors to transmit images to a central computer (at the TMC). The central computer can then transmit images and information to travelers. Components of our system can be modified to work with a centralized network. The 3D compression algorithm can be used to reduce the size of the image transmitted over the network. If the image sensors have processors, the 3D compressed images can 
be processed on the image sensors and both the images and content information can be sent to the TMC. In the case of image sensors without processors, 2D compressed images can be sent to the TMC to be processed. Requests for information and/or images will be made directly to the TMC from travelers. With the centralized network, mobile agents can be used for communication from the travellers to the TMC. The mobile agents can have user specific goals and gather images and information from the TMC. In a centralized network, the TMC requires a large bandwidth to handle the incoming and outgoing images and information. This network is expensive and produces a bottleneck at the TMC that reduces the performance of the system. A distributed network will reduce the probability of bottlenecking and improve the system's overall performance.

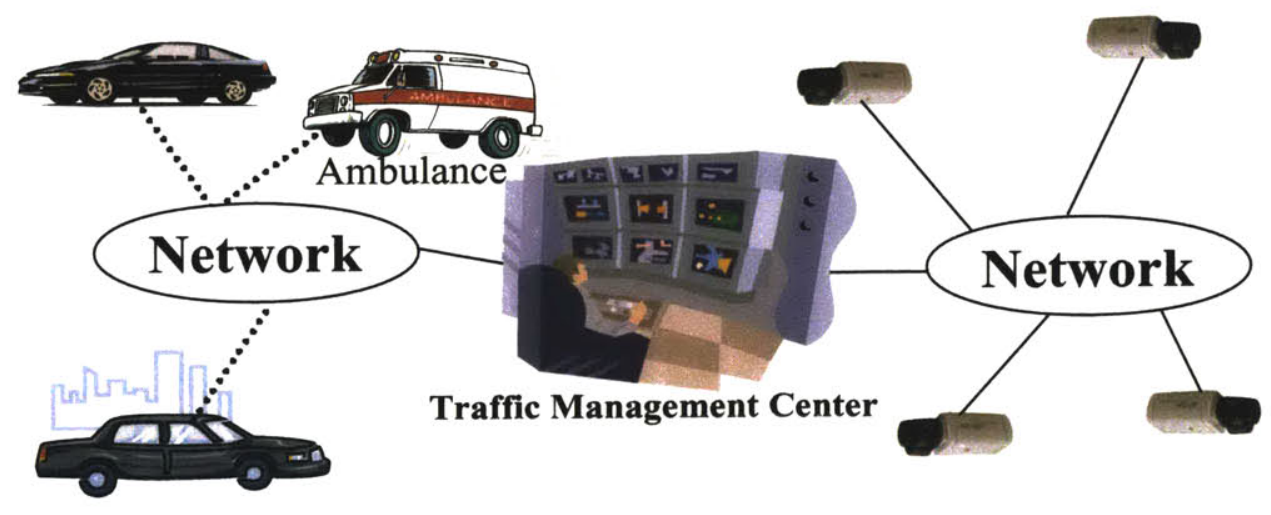

Figure 3-3: Centralized Network Topology 


\section{Chapter 4}

\section{Compression}

A main goal of our system is to process compressed image data. We have designed a compression method that preserves features that are used in image processing. These features are the basis of the compressed data and enable further processing of the image data without decompressing the image. This chapter describes our compression algorithm with performance analysis and comparison with JPEG.

Compression reduces the size of the data by converting the data from one representation to another. A representation is a description of the data. For example, a 5 $\times 5$ binary image as in Figure 4-1(a) can be represented by a matrix of intensities ( 0 , black pixels, and 1, white pixels) shown in Figure 4-1(b). There are 25 intensity values in the matrix, each value needs 1 bit to represent it, therefore 25 bits are needed to represent the matrix of intensities. Run-length coding is a string of numbers, each number is the amount of continuous pixels (pixels that are adjacent by scanning line by line) with the same intensity. In Figure $4-1(\mathrm{c})$, the run-length coding is 6127 , scanning left to right and starting with the number of ones, the code represents 6 
ones followed by 12 zeros and 7 ones. The maximum number of continuous pixels possible is 25 ( 5 bits), using 5 bits for each number in the run-length coding requires 15 bits to encode the binary image. Both the matrix of intensities and run-length coding represent the binary image in Figure 4-1(a), run-length coding requires fewer bits than the matrix of intensities (15 bits for run-length coding compared to 25 bits for the matrix of intensities).

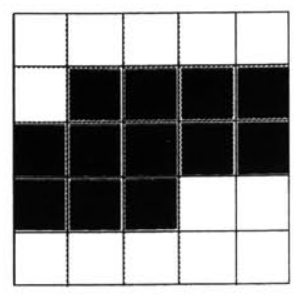

(a) Original Image

\begin{tabular}{|l|l|l|l|l|}
\hline 1 & 1 & 1 & 1 & 1 \\
\hline 1 & 0 & 0 & 0 & 0 \\
\hline 0 & 0 & 0 & 0 & 0 \\
\hline 0 & 0 & 0 & 1 & 1 \\
\hline 1 & 1 & 1 & 1 & 1 \\
\hline
\end{tabular}

(b) Matrix of Intensities (c) Run-length Coding

Figure 4-1: Representation of Data

Compression algorithms encode the data into a new representation. Generally, this new representation will use fewer bits than the original. A given representation may not be suitable for all images. It is possible that while some images require fewer bits, using the same representation for other images may result in an increase in the number of bits. For example in the case of run-length coding, if the $5 \times 5$ binary image is a salt and pepper image (where upon scanning each pixel switches from black to white) the encoding would be 25 ones, requiring 125 bits to encode compared to 25 bits for the matrix of intensities.

Decompression is the process of returning the data to its original representation. Lossless compression fully describes the data with the new representation, and allows us to recover the original data exactly. If, after compression, the new representation 
does not fully describe the data, then the original data cannot be recovered exactly through decompression (lossy compression).

Lossy methods increase image compression ratios at the cost of quality of the decompressed image. This tradeoff between restoring the original data and compression must be considered when deciding on compression methods. The compression method presented here focuses on maintaining attributes of the image that are important for further processing. We chose a lossy compression method which retains edge, average color, motion, and depth information.

Conventional compression schemes such as JPEG (Joint Photographic Experts Group), designed for images that are to be viewed when decompressed, are spatialfrequency based, and are not suitable for use with many image processing algorithms. The goal of our compression algorithm is two-fold; we compress the data as much as possible; and secondly we aim to retain information of importance for object detection. We avoided segmenting the image before compression to provide more flexibility for applications. We modified Marcelo Mizuki's algorithm [56], a contour based lossy compression method. We chose Mizuki's algorithm because it is contour based providing a good foundation for further processing and a low bit-rate design that produces a high compression. Mizuki developed a 2D compression algorithm, we extended to 3D in this research. We have also modified Mizuki's contour coding component to increase compression and maintain connectivity of the contour as well as change the motion component to be contour based. Our method produces a high compression ratio, yet provides for good object detection.

Many image processing schemes $[10,25,33,53,55,58]$ use edge information as a 
basis for object detection, for this reason we chose a contour based compression algorithm to preserve edge information. The contours provide a skeleton of the contents of the image that is used for detection of objects and also provides the basis of our compression, enabling processing of compressed image data to benefit from previous object detection research.

Marshall [51] uses a contour based compression method for shape recognition. Marshall focuses on low resolution images, assuming an object is a long closed contour. In higher resolution images, this assumption creates a problem, since, an object may consist of many disconnected contours. To overcome this problem we allow objects to consist of multiple contours, contours are grouped together to form an object. With low resolution images, Marshall also experienced difficulties with occlusion (overlapping objects in the image). We add three-dimensional information and motion to help separate partially occluded objects. Marshall's work demonstrates the potential of a contour based compression used for detection and recognition. We extend this work, by encoding distance and motion information in the compressed data and removing restrictions to handle high resolution images.

\subsection{Image Compression Method}

Our compression algorithm produces $3 \mathrm{D}$ contours of the original image. The contours are the basis of the compressed image data. The algorithm begins with an edge map. An edge map represents information that describes both the horizontal and vertical edges of an image. Generally, a contour consists of connected edges. Contours are 
found by tracing along the edges of the edge map. The components of the compression algorithm are shown in Figure 4-2. The remaining components use the resulting contours to code the image.

Once the contours are extracted using edge tracing, the contours are coded using our modified differential chain coding. The compressed data contains motion vectors, depth and color for each contour. The distance coding reduces the distance information on the edges of a contour to one average distance value per contour. Color is extracted around the contour and an average color on each side of the contour is coded. The motion vector of each contour is determined using normalized correlation in the area surrounding the centroid of the contour. The colors between contours are found by linear interpolation. To improve the decompressed images, mean block colors between edges can be used along with the linear interpolation. Huffman coding

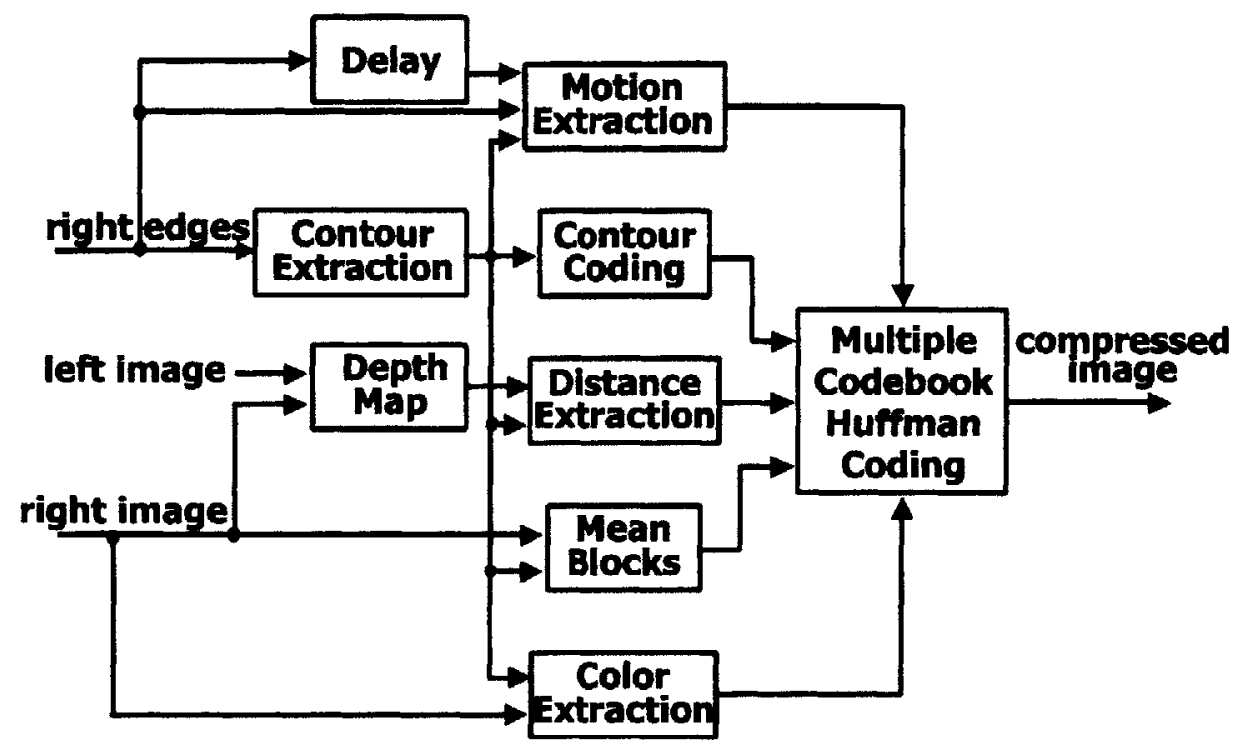

Figure 4-2: Compression Algorithm

is used to further compress the data. Each of the components mentioned here, are described in further detail in the following sections. 


\subsubsection{Depth Map}

The Depth Map module produces an edge depth map that consists of the distances of the vertical edge pixels. The depth map is calculated using a stereo vision algorithm. The stereo vision algorithm performs calculations by determining correspondence of features. Finding the correlation of specific features is faster than finding the correlation at every pixel.

The images are acquired by both frame grabbers simultaneously as described in Chapter 3. Vertical edge gradients of each image are found to sub-pixel accuracy. Corresponding points in the left and right images are found using normalized correlation and the depth is calculated from the corresponding points. Details of the camera calibration and edge based stereo vision algorithm can be found in Chapter 5 .

\subsubsection{Contour Extraction}

A contour is a chain of connected edges. Contour extraction is the process of finding the contours in the image. Contour extraction begins by thinning the edges. Thinning is accomplished by reducing the edges to a pixel width of one. The thinning algorithm uses the mask in Figure 4-3 to determine which pixels to eliminate. Using the gradient information, the center pixel remains if one of the following conditions hold:

- $C$ is a local maximum: $C>U$ AND $C \geq D$ AND $C>L$ AND $C \geq R$ (greater gradient value being at most equal to two mutually orthogonal and adjacent gradients)

- $C$ is a maximum in the horizontal direction: $C>L$ AND $C \geq R$ AND $\left|G_{x}\right|>$ $\left|G_{y}\right|-\alpha$ (greater gradient value being at most equal to one adjacent gradients) 
- $C$ is a maximum in the vertical direction: $C>U$ AND $C \geq D$ AND $\left|G_{y}\right|>$ $\left|G_{x}\right|-\alpha$ (greater gradient value being at most equal to one adjacent gradients)

\begin{tabular}{|l|l|l|}
\hline & $\mathrm{U}$ & \\
\hline $\mathrm{L}$ & $\mathrm{C}$ & $\mathrm{R}$ \\
\hline & $\mathrm{D}$ & \\
\hline
\end{tabular}

Figure 4-3: Thinning Mask

The contours are then obtained by scanning the image from left to right and finding an edge. Figure 4-4 shows a flow chart of Mizuki's contour coding process. After thinning, the edge is traced to produce the contour. If the contour length is greater than $t h_{\text {maxlen }}$, during tracing, the contour is complete and coded. The maximum length threshold is to limit the bit size of the contour length in the code. The contour is also coded if it is longer than the minimum length threshold, $t h_{\text {minlen }}$. This thresholding operation is used to eliminate short contours which are typically due to noise, or contours that do not significantly effect the image (such as from textures).

\subsubsection{Contour Coding}

Mizuki coded the contour using differential chain coding (DCC). DCC is coded by beginning with coding the start location (first edge pixel) followed by the length of the chain (contour) and the direction of each edge pixel in the contour. The direction of each edge pixel is set relative to the previous edge pixel. In differential chain coding (DCC), the code mask changes based on the position of the previous pixel. The code numbering starts clockwise from the previous edge direction. In Figure 4-5, $p$ denotes the previous edge pixel, $c$ denotes the current edge pixel and the code determines the 


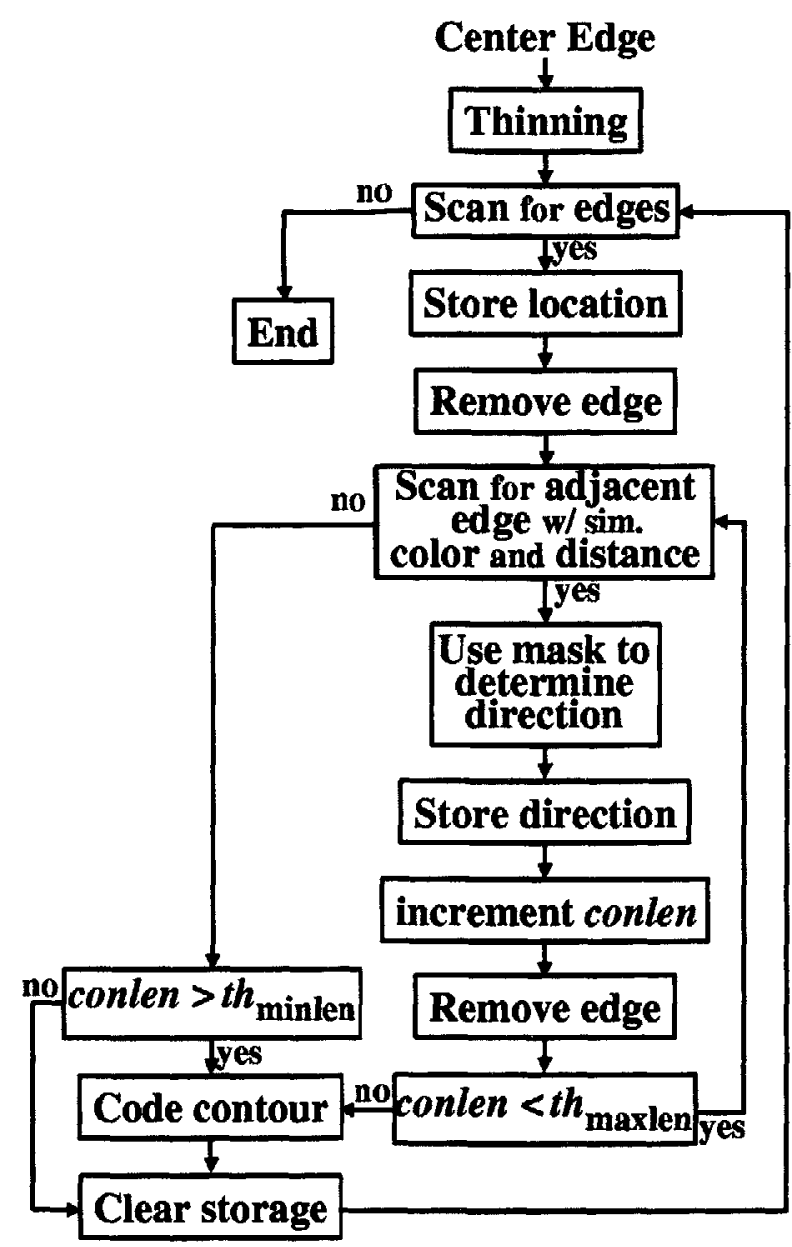

Figure 4-4: DCC Contour Coding Flow Chart

next edge pixel in the chain. For the first code the previous direction can be any preset direction we choose it to be, such as the left of $c$ as in Figure 4-5(a).

If the contour of an object branches, the conventional differential chain coding method will divide the contour into several separate contours. Branching occurs when a line splits into two or more lines. Using the eight nearest neighbor representation of pixels and thinned edges (one pixel width), the number of possible branching configurations is seven for each direction. Figure 4-6 displays all the possible branching configurations for one direction, where the previous pixel is in the upper left. A modification to DCC can be made to include all of the branching, producing an 


\begin{tabular}{|l|l|l|}
\hline 0 & 1 & 2 \\
\hline$p$ & $c$ & 3 \\
\hline 6 & 5 & 4 \\
\hline
\end{tabular}

(a)

\begin{tabular}{|l|l|l|}
\hline 4 & 5 & 6 \\
\hline 3 & $c$ & $p$ \\
\hline 2 & 1 & 0 \\
\hline
\end{tabular}

(e)

\begin{tabular}{|l|l|l|}
\hline 3 & 4 & 5 \\
\hline 2 & $c$ & 6 \\
\hline 1 & 0 & $p$ \\
\hline
\end{tabular}

(f)

\begin{tabular}{|l|l|l|}
\hline 6 & $p$ & 0 \\
\hline 5 & $c$ & 1 \\
\hline 4 & 3 & 2 \\
\hline
\end{tabular}

(c)

\begin{tabular}{|l|l|l|}
\hline 2 & 3 & 4 \\
\hline 1 & $c$ & 5 \\
\hline 0 & $p$ & 6 \\
\hline
\end{tabular}

(g)

\begin{tabular}{|l|l|l|}
\hline 5 & 6 & $p$ \\
\hline 4 & $c$ & 0 \\
\hline 3 & 2 & 1 \\
\hline
\end{tabular}

(d)

Figure 4-5: Direction Codes
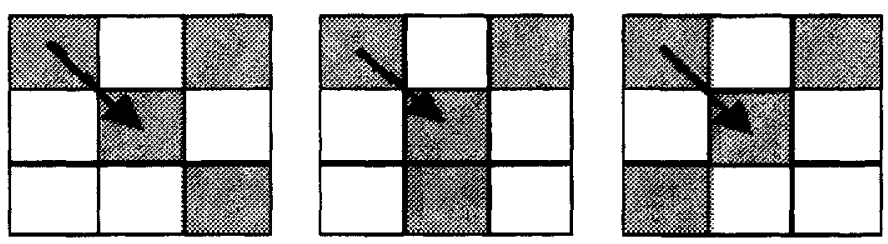

\begin{tabular}{|l|l|l|}
\hline 1 & 2 & 3 \\
\hline 0 & $c$ & 4 \\
\hline$p$ & 6 & 5 \\
\hline
\end{tabular}

(h)
)

.
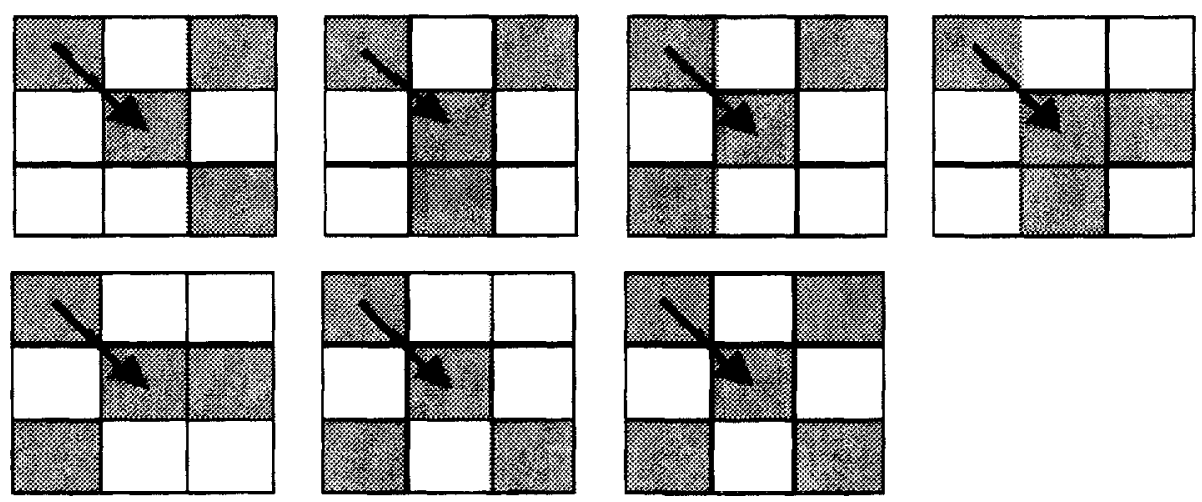

Figure 4-6: Branching Configurations

improved contour representation. When a branch occurs, the modification consists of additional coding and decoding. The contour coding method is modified by providing a depth first contour coding to encode splits, a flow chart is shown in Figure 4-7. The additional codes consists of

\section{7 split escape code}

0 split location marker

1 return to split location.

The 7 is an escape code that specifies a split operation (mark or return), the next code will be a 0 or 1.0 if it is the start of a split, and 1 if returning to the previous 


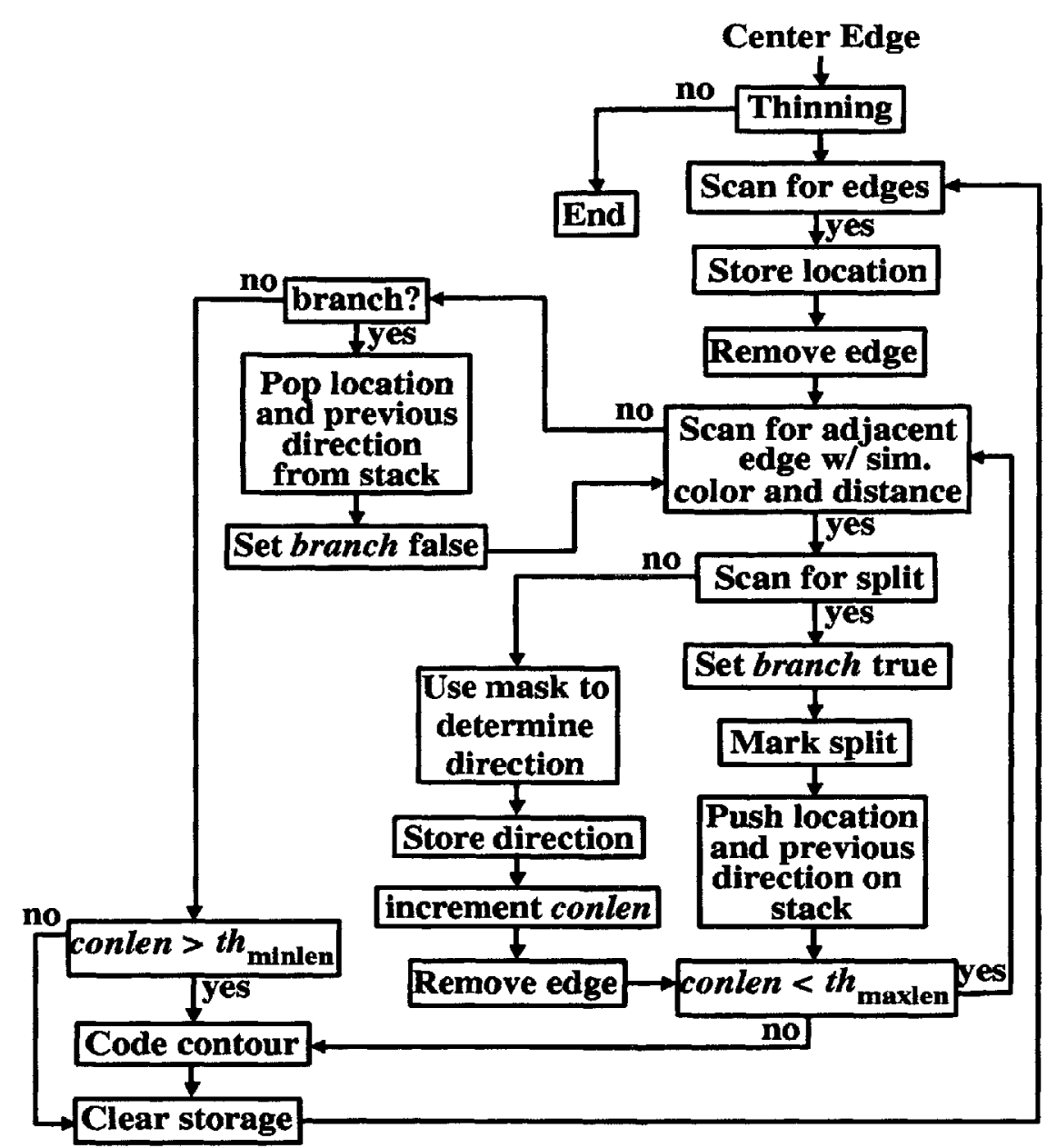

Figure 4-7: MDCC Contour Coding Flow Chart

split. Coding the splits requires keeping track of the split location and previous pixel location. This information is stored in a stack. Stack size is limited by the contour length, avoiding stack overflow. The maximum contour length $\left(t h_{\text {maxlen }}\right)$ is an algorithm parameter that is preset. Incorporating splits eliminates the need for additional start locations at each split. This modification to DCC increases compression, since, in typical size images, coding of a start location requires more bits than coding a split.

If Figure 4-8 is a part of a larger image, the image is scanned and the first edge pixel encountered is at $(101,100)$. Using the code masks shown in Figure 4-5, we 


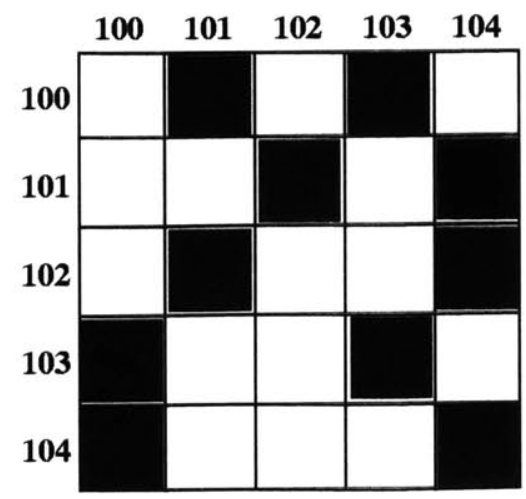

Figure 4-8: Example Contour

consider the DCC (differential chain coding) method. The direction of the next edge is diagonally down to the lower right (the edge located at $(102,101)$ ) which corresponds to the direction indicated by 4 in Figure 4-5(a), the first code mask. The mask in Figure 4-5(b) is now used to determine the next code. There is a choice for the following edge pixel, $(103,100)$ or $(101,102)$, we arbitrarily choose $(103,100)$ in direction 1. The next edge pixel at $(104,101)$ would follow with code 5 using Figure 4-5(h). The contour ending with $(104,104)$ would then be coded as

$$
\begin{aligned}
& \begin{array}{lllllllll}
s_{x} & s_{y} & \mathbf{c} & \mathrm{d} & \mathrm{d} & \mathrm{d} & \mathrm{d} & \mathrm{d} & \mathrm{d}
\end{array} \\
& \begin{array}{lllllllll}
101 & 100 & 6 & 4 & 1 & 5 & 4 & 4 & 1
\end{array} \\
& s_{x} \text { and } s_{y} \text { start location } \\
& \text { c number of direction codes } \\
& \mathrm{d} \text { direction code }
\end{aligned}
$$

where the first two numbers correspond to the start location of the contour, the third number, $\mathbf{6}$, specifies the number of direction codes (contour length) that follow (required for decoding DCC), and the remaining numbers refer to the direction of the pixels in the contour. The edge pixels at $(101,102),(100,103)$, and $(100,104)$ would be coded as a separate contour, as 


\begin{tabular}{|c|c|}
\hline code & bits \\
\hline$s_{x}$ & 7 \\
\hline$s_{y}$ & 7 \\
\hline c & 4 \\
\hline $\mathrm{d}$ & 3 \\
\hline \hline$\underline{\mathrm{e}}$ & 3 \\
\hline$\underline{\mathrm{m}}$ & 1 \\
\hline
\end{tabular}

Table 4.1: Number of bits required for codes in DCC and MDCC

\begin{tabular}{|c|c|c|}
\hline code & \# of code & \# bits to encode \\
\hline$s_{x}$ & 2 & $2 \times 7=14$ \\
\hline$s_{y}$ & 2 & $2 \times 7=14$ \\
\hline $\mathrm{c}$ & 2 & $2 \times 4=8$ \\
\hline $\mathrm{d}$ & 8 & $8 \times 3=24$ \\
\hline \hline Total & \multicolumn{2}{|c|}{$14+14+8+24=60$} \\
\hline
\end{tabular}

Table 4.2: Number of bits to encode example in DCC

$$
\begin{array}{ccccc}
s_{x} & s_{y} & \mathbf{c} & \mathrm{d} & \mathrm{d} \\
101 & 102 & 2 & 6 & 2
\end{array}
$$

Therefore, the entire code using DCC for the example in Figure 4-8 is

$$
\begin{array}{cccccccccccccc}
s_{x} & s_{y} & \mathrm{c} & \mathrm{d} & \mathrm{d} & \mathrm{d} & \mathrm{d} & \mathrm{d} & \mathrm{d} & s_{x} & s_{y} & \mathbf{c} & \mathrm{d} & \mathrm{d} \\
101 & 100 & 6 & 4 & 1 & 5 & 4 & 4 & 1 & 101 & 102 & 2 & 6 & 2
\end{array}
$$

The number of bits required to encode the contours in DCC are dependent upon image size and algorithm parameters. A start location requires 14 bits, assuming the image is $128 \times 128$. The number of bits for contour length is fixed by the algorithm, in this case 4 bits allows up to 15 direction codes. There are 6 possible directions for each mask, requiring 3 bits to encode each direction. The example in Figure 4-8 needs 60 bits to encode, determined from the codes in Table 4.1 and total calculated in Table 4.2 .

Next consider our modified DCC (MDCC) method. We start just as in DCC at 
the start location $(101,100)$, the first code from the mask in Figure 4-5(a) is 4 . Now at a split, $(102,101)$, the split escape and split location marker are coded with 70 . The locations of the split and the previous edge are pushed on the stack to return to the split and use the appropriate mask for coding. The mask in Figure 4-5(b) is used to encode the next direction, the choice of which direction to encode (two choices, $(103,100)$ and $(101,102))$ is arbitrary, for comparison we choose the same direction as DCC $(103,100)$. The remainder of the branch is encoded just as in DCC. After completely tracing one branch, the split escape and return to split location are coded with 71 . The split and previous edge locations are popped from the stack to encode the second branch. The same mask, Figure $4-5(\mathrm{~b})$, is used, starting at the split location $(102,101)$, to encode $(101,102)$ with 5 . The edge pixels $(100,103)$ and $(100,104)$ are coded with 3 and 2, using the mask in Figure 4-5(d). The contour code is

$\begin{array}{cccccccccccccccc}s_{x} & s_{y} & \mathrm{c} & \mathrm{d} & \underline{\mathrm{e}} & \underline{\mathrm{m}} & \mathrm{d} & \mathrm{d} & \mathrm{d} & \mathrm{d} & \mathrm{d} & \underline{\mathrm{e}} & \underline{\mathrm{m}} & \mathrm{d} & \mathrm{d} & \mathrm{d} \\ 101 & 100 & \mathbf{9} & 4 & \underline{1} & \underline{0} & 1 & 5 & 4 & 4 & 1 & \underline{7} & \underline{1} & 5 & 3 & 2\end{array}$

e split escape

m split location or return marker

which requires 53 bits, shown in Table 4.3 (14 for the start location, 4 for the number of direction codes, 27 for direction codes and 8 for the split operations). In this example, DCC requires 7 more bits than our $\mathrm{MDCC}$ to encode the same information. The encoding start location and contour length for the second branch in DCC is greater than the encoding of the direction and additional bits needed to encode the split in MDCC. 


\begin{tabular}{|c|c|c|}
\hline code & \# of code & \# bits to encode \\
\hline$s_{x}$ & 1 & $1 \times 7=7$ \\
\hline$s_{y}$ & 1 & $1 \times 7=7$ \\
\hline $\mathbf{c}$ & 1 & $1 \times 4=4$ \\
\hline $\mathrm{d}$ & 7 & $9 \times 3=27$ \\
\hline$\underline{\mathrm{e}}$ & 2 & $2 \times 3=6$ \\
\hline$\underline{\mathrm{m}}$ & 2 & $2 \times 1=2$ \\
\hline \hline Total & \multicolumn{2}{|c|}{$7+7+4+27+6+2=53$} \\
\hline
\end{tabular}

Table 4.3: Number of bits to encode example in MDCC

To determine the effect of incorporating splits on the compression ratio, consider compressing a contour that splits $m$ times, (where each split divides into two parts) and the entire contour including all splits consists of $n$ pixels. In differential chain coding (DCC), each split represents a new chain and the start locations of each must be encoded. There are $m$ splits, therefore, there are $(m+1)$ starting locations and contour lengths to be encoded. Along with the starting locations, the remainder of the contour must be encoded. There are $(n-(m+1))$ pixels to be encoded. Using DCC, the number of bits to code a contour is

$$
C_{D C C}=(m+1) s+(m+1) c+(n-m-1) d
$$

where $s$ is the number of bits to code a starting location, $c$ is the number of bits to code a contour length, and $d$ is the number of bits to code a direction. The number of bits to encode our modified differential chain coding (MDCC) is

$$
C_{M D C C}=s+c+(n-1) d+2 m(d+1) .
$$


There is only one start location and contour length to encode, the remaining $(n-1)$ pixels are directions. There are also $2 m$ split operations to encode. Each split escape code is followed by one bit to specify the start or return from a split. MDCC reduces the number of bits per contour, when

$$
\begin{aligned}
C_{D C C} & \geq C_{M D C C} \\
(m+1) s+(m+1) c+(n-m-1) d & \geq s+c+(n-1) d+2 m(d+1) \\
m s+m c-m d & \geq 2 m d+2 m \\
m s+m c & \geq 3 m d+2 m \\
s+c & \geq 3 d+2
\end{aligned}
$$

Since $d=3$ to encode the 6 directions and 1 split escape, MDCC will be more efficient than DCC when $s+c \geq 11$. Figure 4-9 shows the number of bits for each coding scheme versus $s$ (the number of bits needed to code a location in the image). To encode the locations in a $64 \times 64$ image 12 bits are needed. Therefore any image $64 \times 64$ or larger can be encoded with fewer bits using MDCC.

The maximum contour length is a preset variable that limits the number of direction codes in a contour. The maximum number of direction codes is equal to the maximum contour length minus one. The calculations to determine the number of bits to encode a contour in DCC and MDCC assume that the MDCC does not exceed the maximum number of direction codes. For MDCC, contours are extended to include more pixels, the maximum number of direction codes will probably be reached. Once the maximum is reached, a new contour has to be started to complete the coding. For example, if, in the coding of Figure $4-8,7$ is the maximurn number of direction codes in a chain instead of $15, \mathrm{c}$ in Table 4.1 now requires 3 bits instead of 


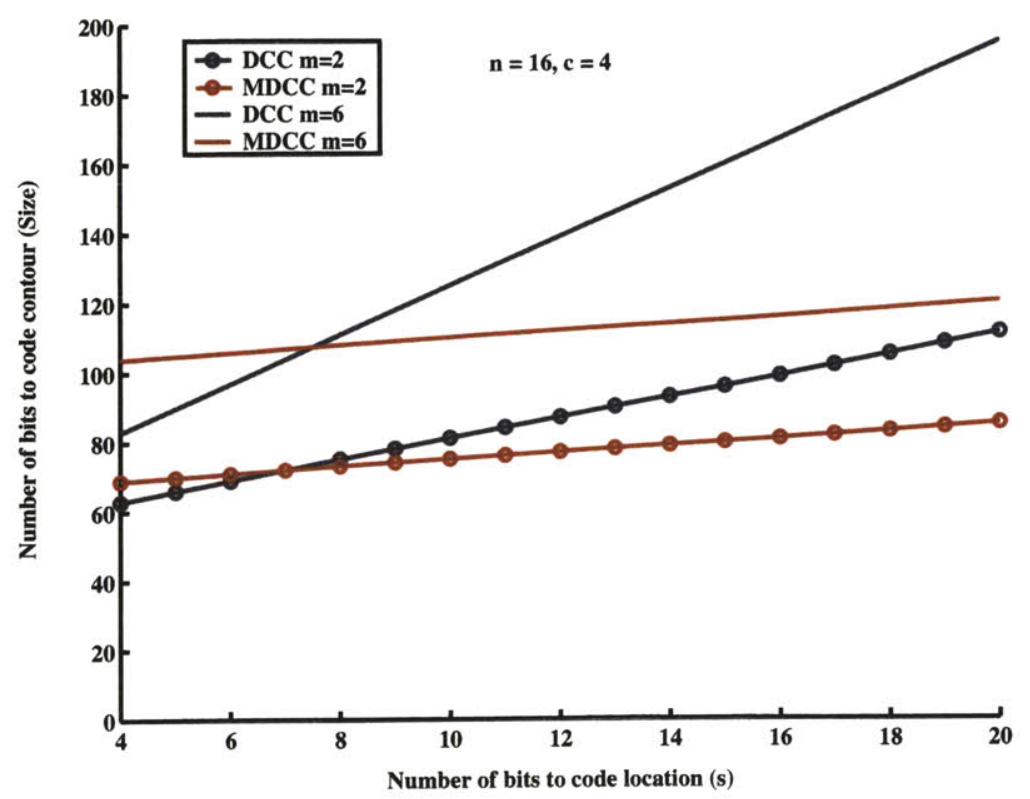

Figure 4-9: Bits to encode a contour

4 bits. The DCC code remains the same but requires 58 bits instead of 60 bits. The MDCC code on the other hand becomes

$$
\begin{array}{llllllllllllllllll}
s_{x} & s_{y} & \mathbf{c} & \mathrm{d} & \underline{\mathrm{e}} & \underline{\mathrm{m}} & \mathrm{d} & \mathrm{d} & \mathrm{d} & \mathrm{d} & \mathrm{d} & \underline{\mathrm{e}} & \underline{\mathrm{m}} & \mathrm{d} & s_{x} & s_{y} & \mathbf{c} & \mathrm{d} \\
101 & 100 & \mathbf{7} & 4 & \underline{7} & \underline{0} & 1 & 5 & 4 & 4 & 1 & \underline{7} & \underline{1} & 5 & 100 & 103 & \mathbf{1} & 5
\end{array}
$$

requiring 66 bits ( 28 for the start locations, 6 for c, 24 bits for the 8 directions, and 8 bits for the split). Since the maximum number of direction codes is reached before the contour is completed, the description contains two contours with the additional bits to mark the position and return of the split. If $r$ splits have been marked and not completed, there are $2 r(d+1)$ additional bits in the MDCC code. Over the entire image these additional bits reduce or eliminate the savings of coding with the split, possibly increasing the number of bits required to code the image. The effect of incomplete contour coding can be reduced by making the maximum number of direction codes large enough, through empirical analysis. Another option is to remove the maximum number of direction codes restriction and to determine the 
maximum after the entire image is coded. This requires the entire image to be coded before transmission. In section 4.3, we look at different cases for setting the maximum number of direction codes to 16,32 , and 64 .

\subsubsection{Distance Extraction}

Our compression method is contour based, we assign one distance value for the entire contour. The average distance of the pixels in the contour is used as the distance for the contour. The pixels of a contour are grouped by proximity, color, and distance, therefore the variation in distance of the contour is dependent on the similarity metric and threshold used to generate the contour.

\subsubsection{Color Extraction}

Color extraction applies to the average color on each side of the contour. The number of bits needed for each color is reduced by color quantization. There are many methods for quantization (representing $256^{3}$ colors with $N$ colors), such as popularity, mediancut, octree, and uniform [85]. The popularity method consists of dividing the colors into bins and picking the average of the $N$ most popular bins, colors not in the bins are mapped to the closest color. Median-cut finds the color mapping such that each of the $N$ colors represents the same number of colors, and octree divides the colors into a $\log N$ depth tree where each leaf represents an average of several colors. The method used in this system is a simple uniform color quantization. The colors are uniformly divided based on the number of bits desired. This method does not produce the best 
results for maintaining color, a better method can be used at the cost of increased complexity.

The contour has two sides, each side has a quantized color associated with it. Figure 4-10 shows the colors on both sides of the contours that are extracted. The color is determined by scanning the image from left to right and approximating a line from one edge to the next, then scanning the image from top to bottom and approximating a linear fit from one edge to the next. If a pixel has a horizontal and vertical color associated with it from the scans, the color assigned to the pixel is the average color of the color from the horizontal and vertical scans. Once a color has been assigned to each pixel on the left and right side of the contour, the average along each side is the value which is coded.
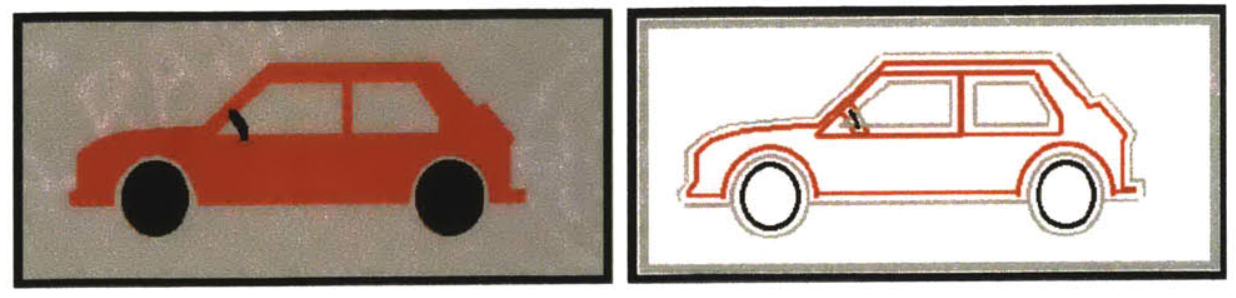

Figure 4-10: Color Extraction
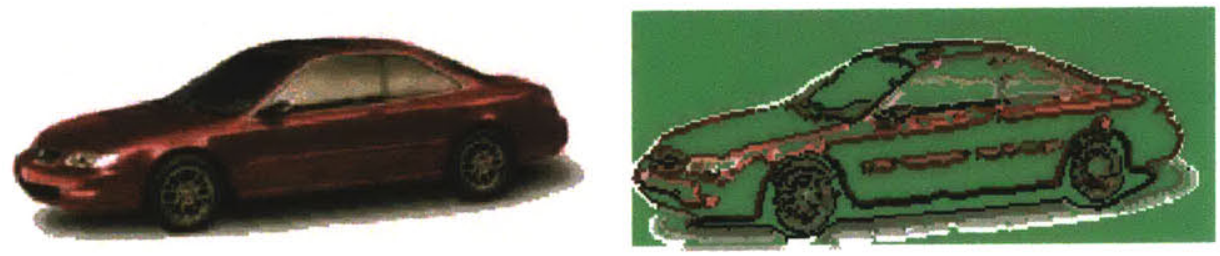

Figure 4-11: Example of Color Extraction 


\subsubsection{Mean Blocks}

A mean block is the average value of an area. The image is divided into blocks that are $20 \times 20$. The average color of each block, which does not contain a contour, is determined. Figure 4-12 shows the area of the mean blocks and Figure 4-13 shows

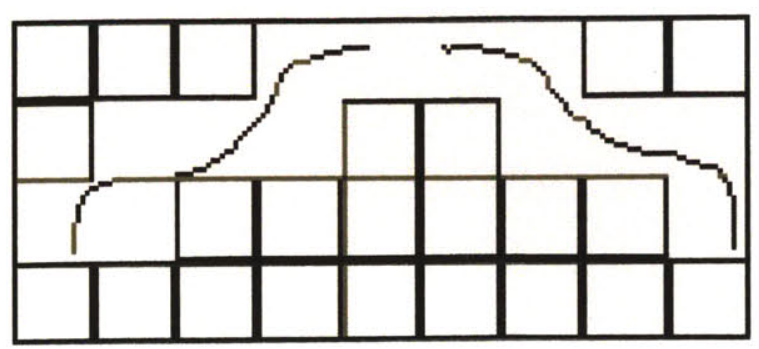

Figure 4-12: Mean Blocks

approximately the values that are coded between contours. The mean blocks are used to improve the quality of the image. Increasing the size of the blocks or eliminating the blocks can increase the compression.
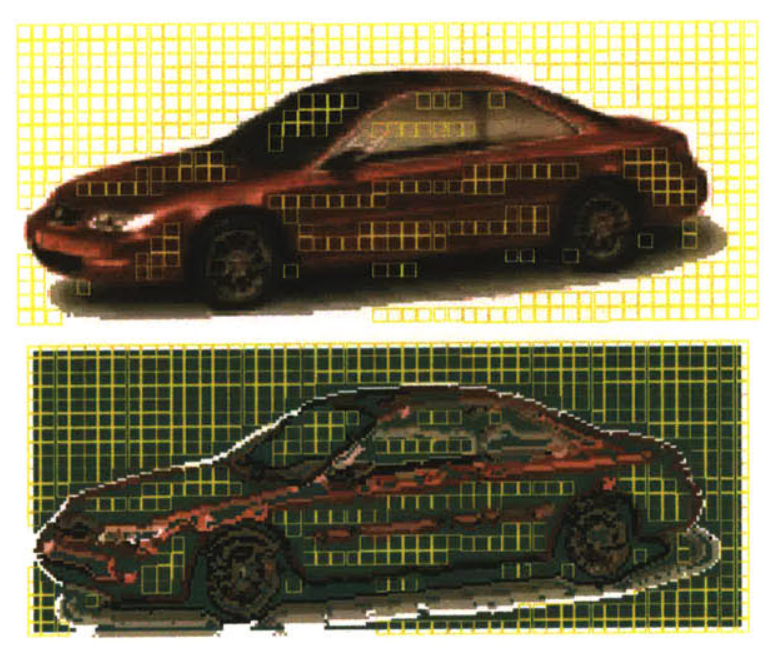

Figure 4-13: Mean Blocks Example 


\subsubsection{Motion Extraction}

Motion is extracted by using a normalized correlation on the area surrounding the contour and searching the subsequent image for the best match. This process is similar to finding the corresponding points in the stereo vision algorithm described in Chapter 5 , the difference being the search region. The search region in the stereo vision algorithm is a line and this search region consists of a large area centered at the previous location of the contour. Reducing the search area will significantly improve computation. The search area can be reduced by any combination of three methods, 1) estimates of object motions based on content (object physical characteristics: rigid body motion, acceleration ranges, lane boundaries, etc.), 2) a hierarchical (multiresolution) search, and 3) predictive searches using previous motion to reduce the search area. Finding the best option to reduce the search region is highly dependent upon the application. We chose to use an exhaustive search to find the best estimate for contour motion, to demonstrate the feasibility of processing compressed image data.

\subsection{Image Decompression}

Decompression is the process of converting (decoding) compressed data into the original representation (decoded image). Our decompression method is lossy, the decoded image is not the same as the original image. To assess lossy compression algorithms, we use several measurements, the compression ratio, root-mean squared error (RMSE), peak signal-to-noise ratio (PSNR), and/or error image. The compression ratio, $c$, is the ratio of the size of the original data to the size of the encoded 
data,

$$
c=\frac{\text { size of original data }}{\text { size of encoded data }}
$$

The encoded data is the newly represented data produced by the compression algorithm.

The root-mean squared error is the error of the decoded image from the original image defined as

$$
R M S E=\sqrt{\sum_{i=1}^{N} \sum_{j=1}^{M} \frac{\left[I_{R}(i, j)-\hat{I}_{R}(i, j)\right]^{2}+\left[I_{G}(i, j)-\hat{I}_{G}(i, j)\right]^{2}+\left[I_{B}(i, j)-\hat{I}_{B}(i, j)\right]^{2}}{M N}}
$$

where $i, j$ are the pixel coordinates, $M \times N$ is the size of the image, $I_{X}(i, j)$ represents the intensity in channel $\mathrm{X}$ at $i, j$ in the original image and $\hat{I}_{X}(i, j)$ is the intensity in channel $\mathrm{X}$ at $i, j$ in the decoded image. The lower the RMSE, the closer the decoded image is to the original.

PSNR is another commonly used measurement to analyze error, defined as

$$
P S N R=20 \log _{10}\left(\frac{I_{\max }}{R M S E}\right)
$$

where $I_{m a x}$ is the peak signal of the original image and $R M S E$ is the root-mean squared error, defined above, also considered the average noise of the decompressed image. A high PSNR signifies low noise and an image which is closer to the original.

The advantage of the PSNR and RMSE is that by using a single number, we can easily compare compression algorithms. However, the visual comparison of the original and decoded images may provide a better analysis. Assuming two differ- 
ent compression algorithms have the same compression ratio but result in different RMSE values for a given image, the compression algorithm with lower RMSE may be preferred, since the decoded image overall is closer to the original. But, the compression algorithm with a higher RMSE may be better suited for a specific application than a compression algorithm with a lower RMSE. For example, in a video phone, images are compressed before transmission. The periphery of the video phone images is generally not as important, assuming the face is centered in the image. If the compression algorithm with a higher RMSE has large errors on the periphery, while an algorithm with lower RMSE has errors throughout the image, the former algorithm may be better suited for video phone applications. It is not practical to use RMSE or PSNR as the only measure of performance, additional methods of comparison with the original are needed.

The error-image displays visually where the differences between the original and decoded image are most noticeable. An error image for a comparison of images can be generated, for example, by

$$
E_{X}(i, j)=255-\left|I_{X}(i, j)-\hat{I}_{X}(i, j)\right|
$$

where $i, j$ are the pixel coordinates, $I_{X}$ is the intensity in channel $\mathrm{X}$ of the original image, $\hat{I}_{X}$ is the intensity of the decoded image and $\left(E_{R}, E_{G}, E_{B}\right)$ is the error image. For pixels where the original and decoded images are equal, the intensity in the error image will be $(255,255,255)$ which will be white. The error image intensities will range from $(0,0,0)$, completely different, to $(255,255,255)$, no difference. An example of an 
error image is shown in Figure 4-14. The error image, although visual, provides only a very subjective measurement. Ideally using the error image, along with PSNR or RMSE, and the compression ratio currently provide the best overall measurement of performance.
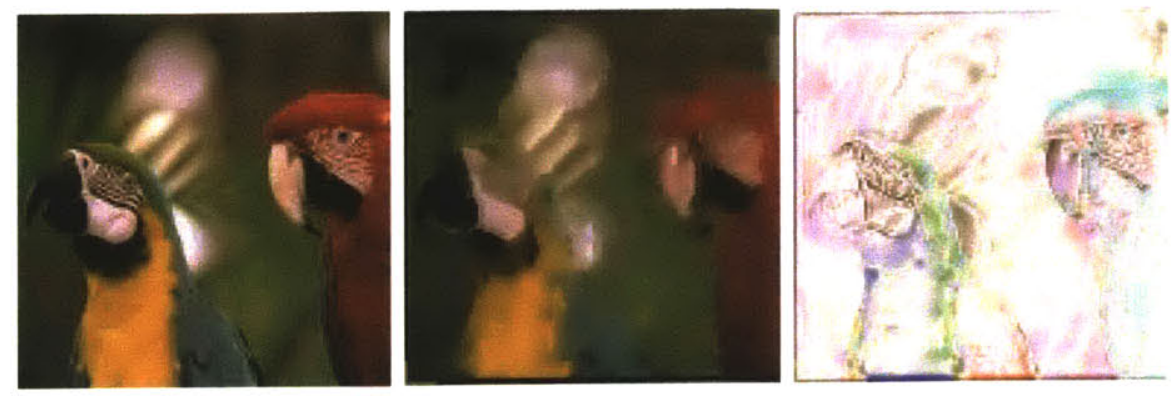

Figure 4-14: Original, Compressed and Error Images

Given the compressed data as input, the multiple codebook Huffman decoder is used to produce contour, distance, motion vector, contour colors, and mean codings that need to be decoded to generate a decompressed image. A contour decoder shown in Figure 4-15 will generate the thinned edge map by determining the edge locations. The colors on the left and right side of the contour are then added to the edge map. The color values between the contours are estimated by linear interpolation from the color information surrounding the contours. Finally, the mean blocks are added between the contours and a smoothing algorithm is used to reduce the blockiness produced by the means. The following sections provide details of the image decompression. 


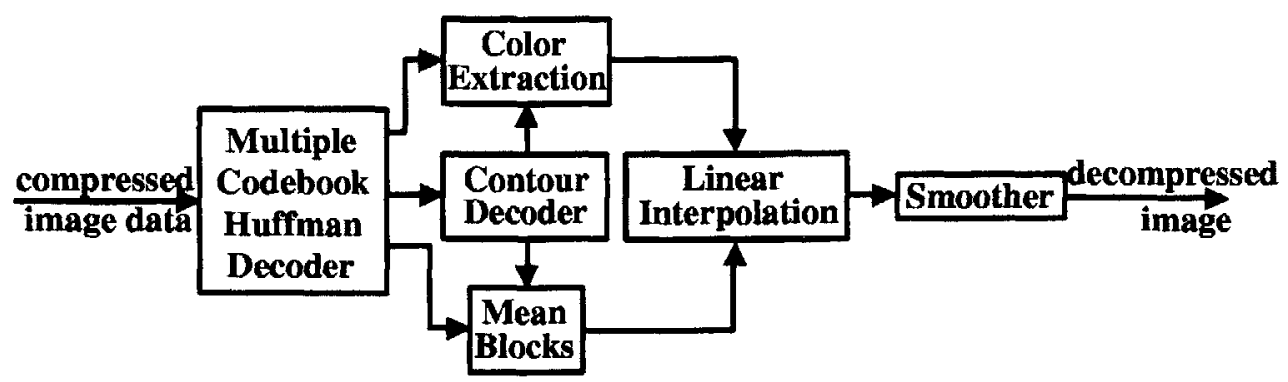

Figure 4-15: Decoder Flowchart

\subsubsection{Contour Decoder}

Given the modified differential chain coding (MDCC) for the entire image, the contour decoder generates an edge image. The following code was encoded with the same parameters as in Table 4.1,

11001011100100100110011100011011001000011111101011010.

The first 7 bits represent the $x$-coordinate of the start location $(1100101 \rightarrow 101)$ and the next 7 bits represent the $y$-coordinate $(1100100 \rightarrow 100)$. The following 4 bits correspond to the number of direction codes that will follow $(1001 \rightarrow \mathbf{9})$. The number of direction codes determines when the contour code ends. The next 9 codes excluding codes related to splits are direction codes.

So far, the highlighted region below has been decoded (18 bits) and the remaining bits needs to be decoded

11001011100100100110011100011011001000011111101011010.

The direction codes are 3 bits each, the next 3 bits $(100 \rightarrow 4)$ is the first direction 
code. The edge pixel coordinate is determined by using the same initial mask as the encoder (Figure 4-5(a)), yielding the pixel at location $(102,101)$. There are now 8 direction codes remaining, the next three bits $(111 \rightarrow 7)$ represents the split escape code (since the direction codes range from 0 to 6 ), the following bit 0 specifies the start of a split. Therefore, a split is located at $(102,101)$. The split location and previous location $(101,100)$ are pushed on a stack for future use. The next three bits $(001 \rightarrow 1)$ give the direction of the next pixel. The mask in Figure 4-5(b) is used based on the previous location $(101,100)$ and current location $(102,101)$ to find the edge pixel at $(103,100)$.

The process continues, looking at the next three bits. If the three bits are 0-6 then it is a direction code and the appropriate mask is used to determine the location of the edge pixel; if the three bits represent a 7 , the next bit specifies the start of a split or the end of a branch. In the example, when the next 7 is reached,

\section{0}

it is followed by a 1 specifying the end of a branch. The split location and previous location are popped from the stack and the remainder of the directions are decoded.

This code yields the example in Figure 4-8. Once all 9 directions have been decoded the next contour is decoded, starting with the start location and number of direction codes. To ensure that there is no ambiguity in the decoding, the code must not end with a split operation $(71(0))$. The number of direction codes determine the end of the code, therefore ending the code with $(71(0))$ a split operation would be 
seen as a part of bits for a start location rather than a split operation. This can be avoided in the encoding stage by removing any code ending with a split operation. Since the operation is at the end of the code this will not effect the code and avoids any ambiguity.

\subsubsection{Color Decoding}

There are two types of color information that need to be decoded. There is the color information for each side of the contour and the mean blocks in between contours. Once a contour has been decoded the color information on each side of the contour is added, producing an image similar to Figure 4-11. The mean blocks are added between the contours followed by linear interpolation.

\subsubsection{Linear Interpolation}

The color information between contours is found using linear interpolation and mean blocks. The color of a pixel that has not been found by the color decoder is found by using linear interpolation. The end points of the line are from colored pixels in both the horizontal and vertical directions. The average of horizontal and vertical linear interpolation for a pixel determine the pixel color. After all pixels have been assigned a color, the image is smoothed to reduce sharp contrasts in color. An example of the effect of smoothing is shown in Figure 4-16. 

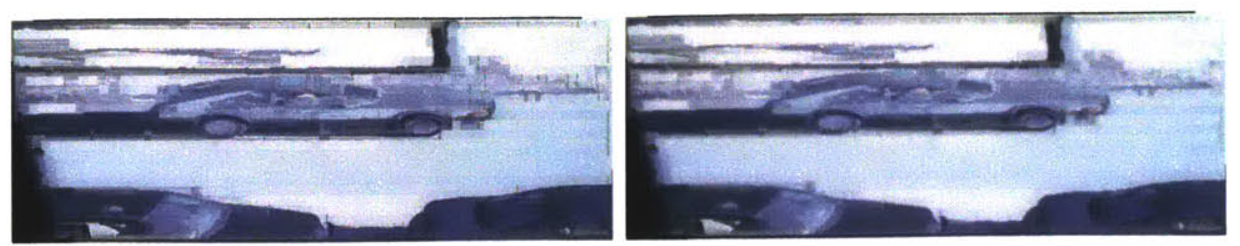

Figure 4-16: Decompressed image before and after smoothing.

\subsection{Compression Results}

Our compression algorithm is design to enable processing of the compressed image data without decompression. We focus on retaining features of the image that are common in image processing algorithms. With this in mind, our compression results focus on retaining edge information and improving the compression ratio. In this section, we show three types of results, 1) a comparison with JPEG demonstrating the retention of edge information, 2) a bit-rate distortion curve of "lena" and "parrots" images, presenting the tradeoff between compression and error, and 3) a comparison of DCC with MDCC showing improved compression using MDCC.

\subsubsection{JPEG Comparison}

JPEG is a lossy compression method designed to provide a high compression. Our method is also designed for high compression with the added benefit of preserving edges from the original image. We compare JPEG with our method by looking at the RMSE and edge image at similar compression ratios.

In both, JPEG and our method, the decompressed images contain artifacts as seen in Figure 4-18. The blocky artifacts in JPEG images alter the edge information, making edge based processing inaccurate. These artifacts also produce errors when 


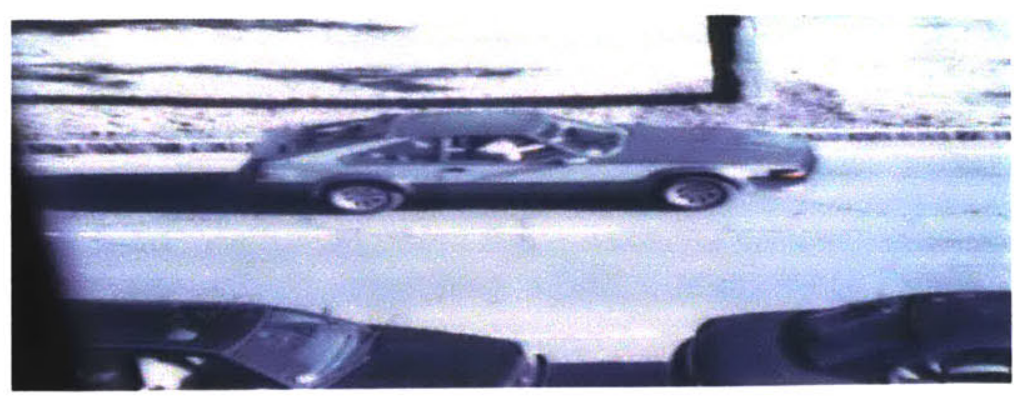

(a) Original Image

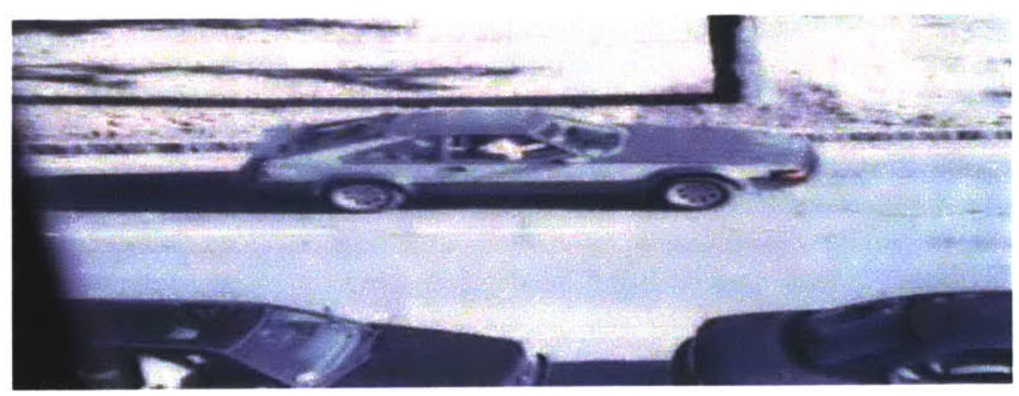

(b) JPEG Image bpp $=0.53$ and RMSE $=36$

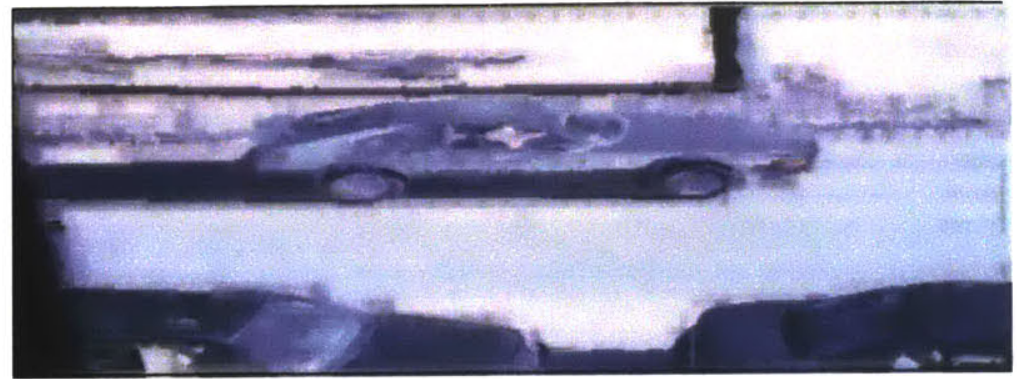

(c) Our Decompressed Image bpp $=0.54$ and RMSE $=43$

Figure 4-17: JPEG Compression Comparison

detecting motion vectors in the decompressed images. Our compression method produces images that are also blocky, but the edge and motion information are available in the compressed image data. Since our compression method is contour based, the edge information is taken from the compressed image data directly, rather than using edge detection on the decompressed image. This gives accurate edge information compared to JPEG, where we need to perform edge detection on the decompressed 


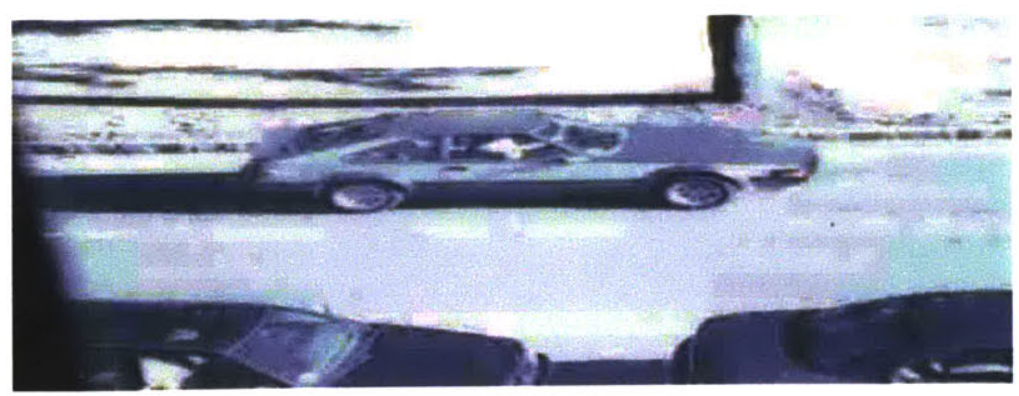

(a) JPEG Image bpp $=0.24$ and RMSE $=40$

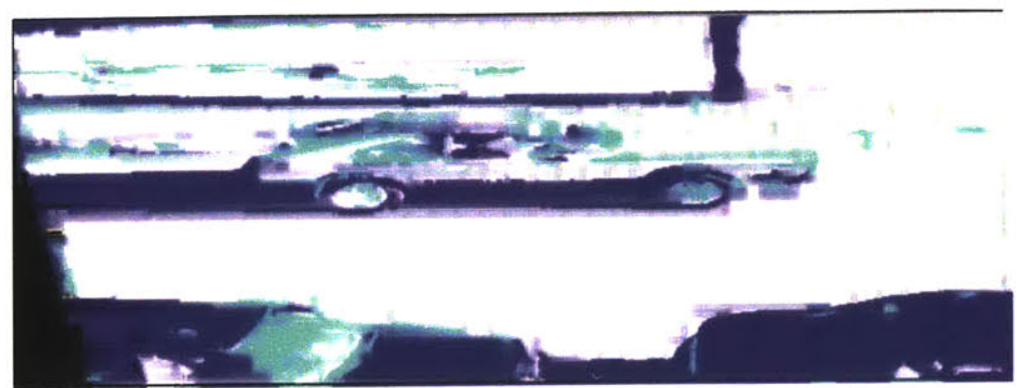

(b) Our Decompressed Image bpp $=0.24$ and RMSE $=88$

Figure 4-18: JPEG Compression Comparison

image.

We use the same edge algorithm on the original image and the compressed images of Figure 4-17 and Figure 4-18. Our edge image (Figure 4-19(c)) differs from the original edge image (Figure 4-19(a)) due to the elimination of small contours (assumed to be noise or texture details), this parameter can be set to include all contours in the compressed image. It is clear, in the case of JPEG, higher compression adds edges (Figure 4-19(d)) that will produce errors in edge based algorithms. Our compression method does not add false edges with higher compression (same edge image for both compressions). 


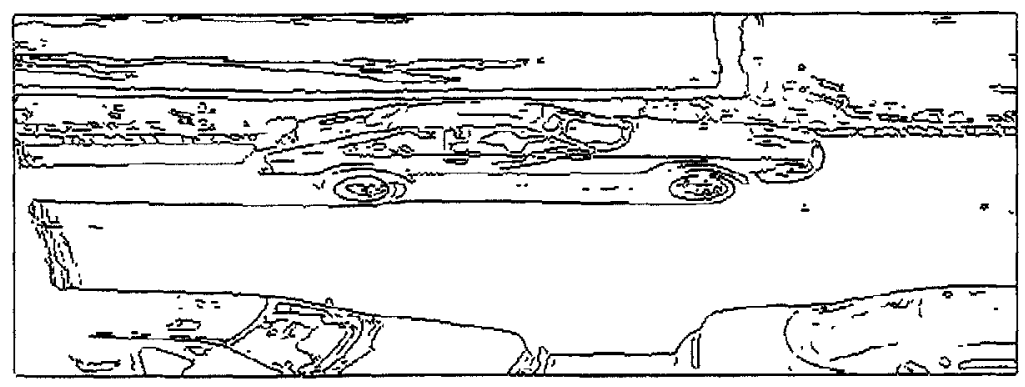

(a) Edge of Original Image

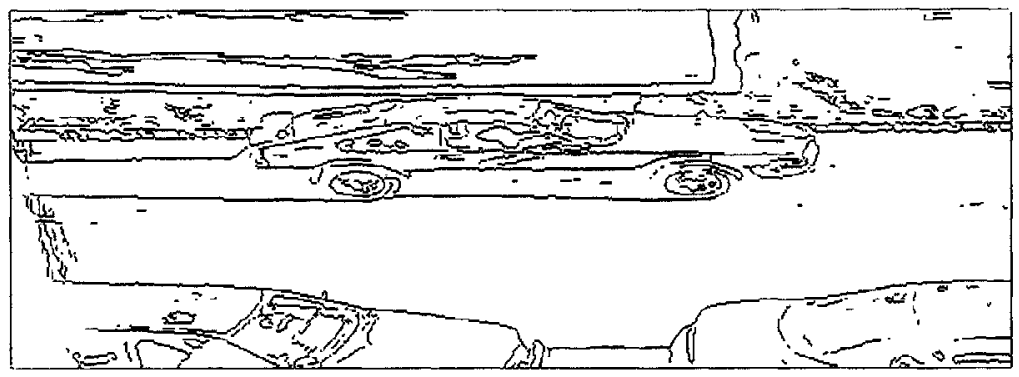

(b) Edge of JPEG Image in Figure 4-17(b)

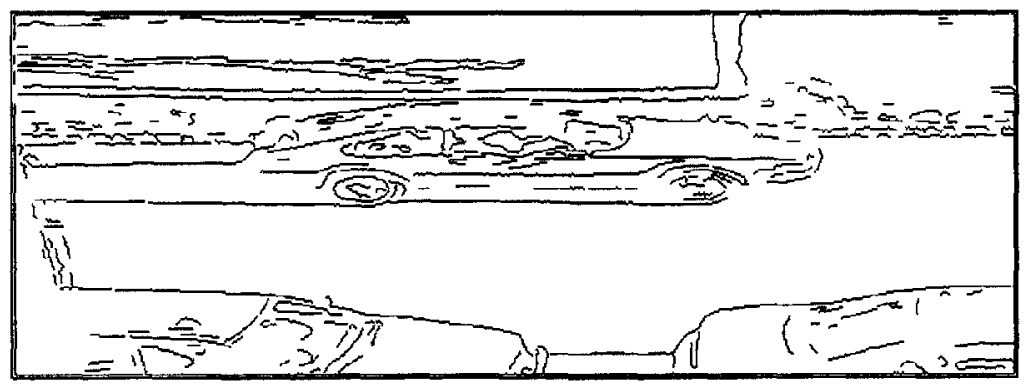

(c) Edge of our images in Figure 4-17(c) and Figure 4-18(b)

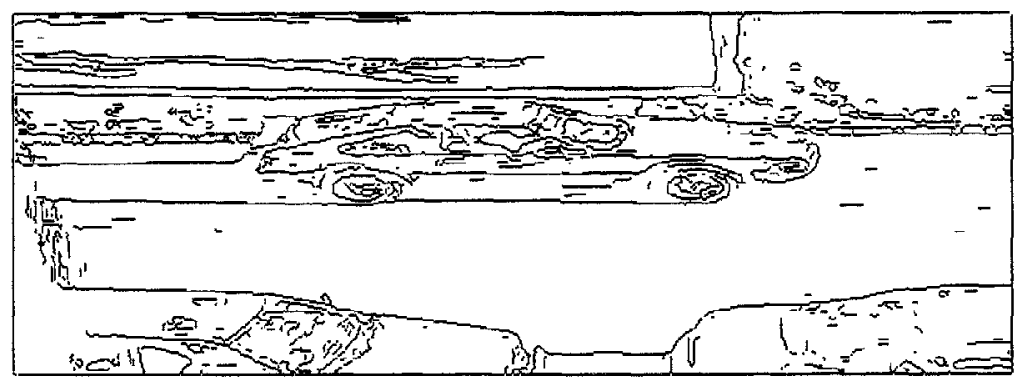

(d) Edge of JPEG Image in Figure 4-18(a)

Figure 4-19: Comparison of Edge Images 


\subsubsection{Bit-Rate Distortion}

Distortion refers to the difference between the original and decompressed images. Although our goal is not to reduce distortion, but to provide a compression method that is conducive to further processing, we generate bit-rate distortion curves for two examples. The bit-rate is measured as bpp (bits per pixel) and the distortion is measured using RMSE, defined in the section 4.2. The curve presents the tradeoff between compression and error, generally the higher the compression, the more error. Figure 4-20 is the bit-rate distortion curve of two common images, "lena" and the "parrots." The parrots have a higher compression with less distortion, primarily due to fewer edges and less textures in the image.

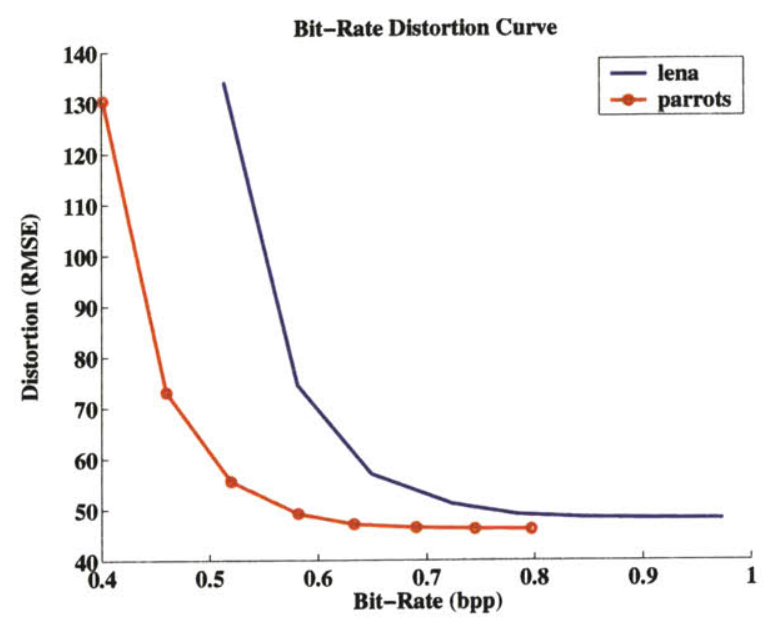

Figure 4-20: Bit-rate distortion curve for "lena" and "parrots" images

\subsubsection{DCC vs. MDCC}

We test our compression algorithm over 6 days on image sequences to determine if the compression ratio is higher using MDCC or DCC. Both full color images and binary edge images are compressed. The image sequences are of a street with traffic 
flowing in one direction as well as some parked vehicles. Ten to thirty sequences are captured each day (image set) at 30 frames/sec in sequences that on average contain 50 frames, for a total of over 6000 images. A sample image and edge map from each day is given in Figure 4-21. The day 1, 2, and 3 (numbered top to bottom) are taken at a higher elevation (approximately $9 \mathrm{~m}$ ), then 4, 5, and 6 (approximately $2 \mathrm{~m}$ ).
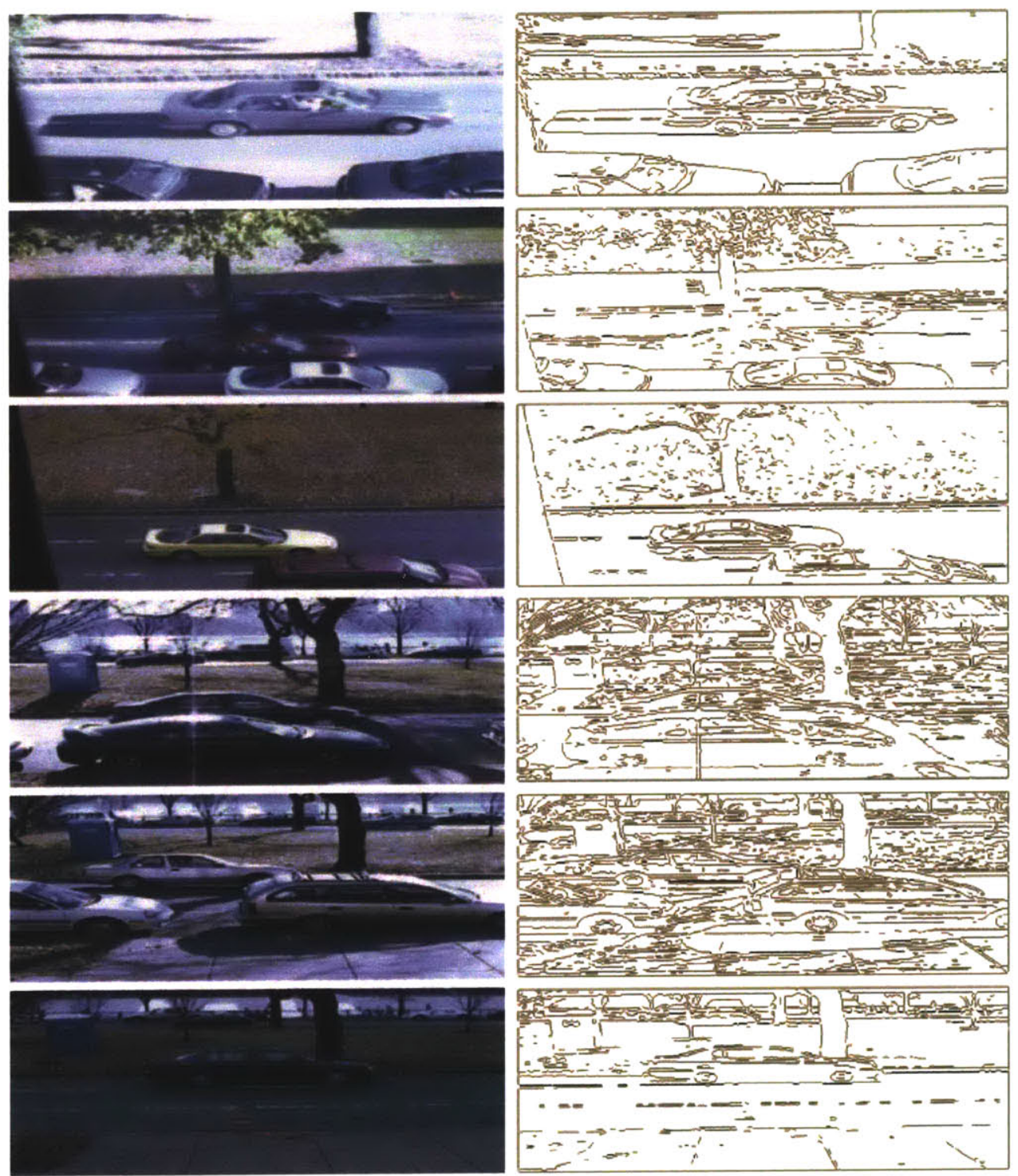

Figure 4-21: Sample color and edge images from each set

In a contour based compression method, the compression ratio is directly related 
to the number of edges in the image. The more edges in an image, the more contours, resulting in a lower compression ratio. Figure 4-22 shows the compression ratios of

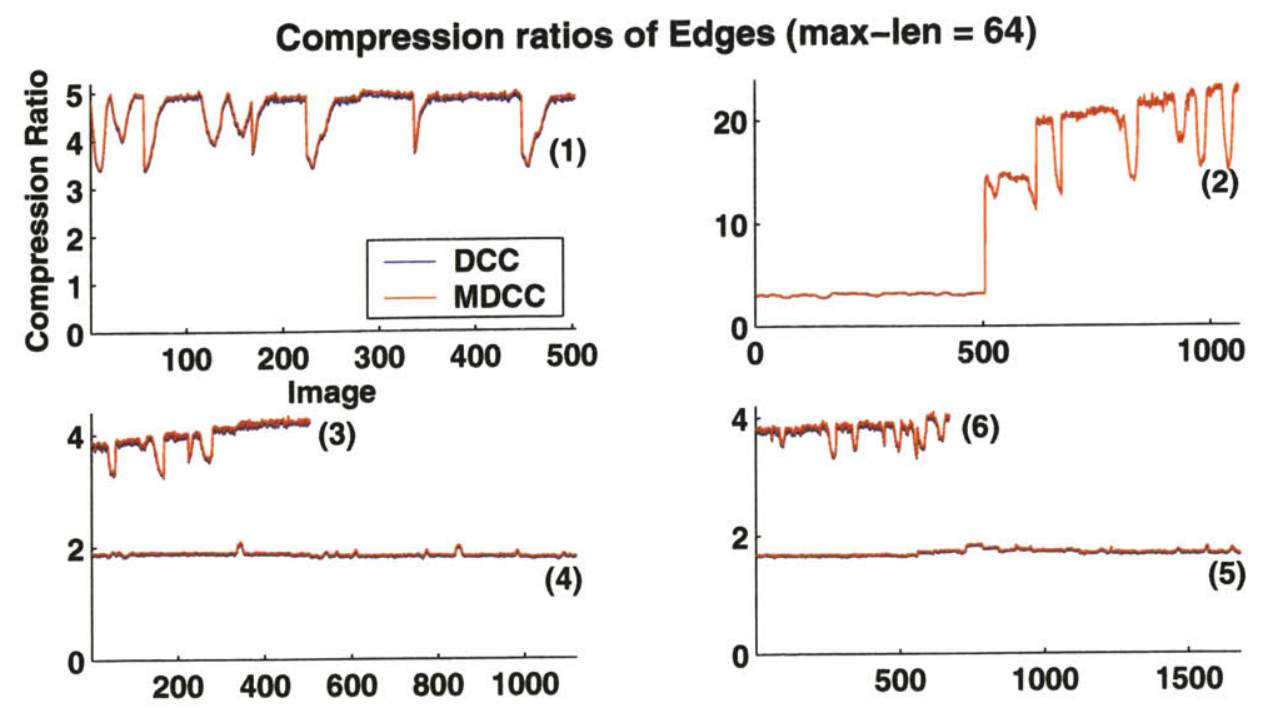

Figure 4-22: Compression Ratio of Edge Images with maximum number of direction codes equal to 64

edge images (for each image set with a maximum number of direction codes of 64) without Huffman coding for both DCC and MDCC. There is a small improvement in using MDCC, most visible in image set 1 . The advantage of MDCC is fully realized when there are many splits and the number of directions does not exceed the maximum number of direction codes.

The dips in the compression ratio in Figure 4-22 (prominent in image sets 1 and 2) occur when one or more vehicles travel through a range of images. If a vehicle has more edges than the background it is occluding, the compression ratio decreases when a vehicle appears in the scene. Image sets 4 and 5 have a large number of edges seen in Figure 4-21, making the appearance of vehicles insignificant to the overall compression ratio, therefore no major difference in compression ratios is present. The effect of edges can also be seen in the sharp increase in compression ratio in image 
set 2. There is a 1 hour delay between images with the onset of dusk. Figure 4-23
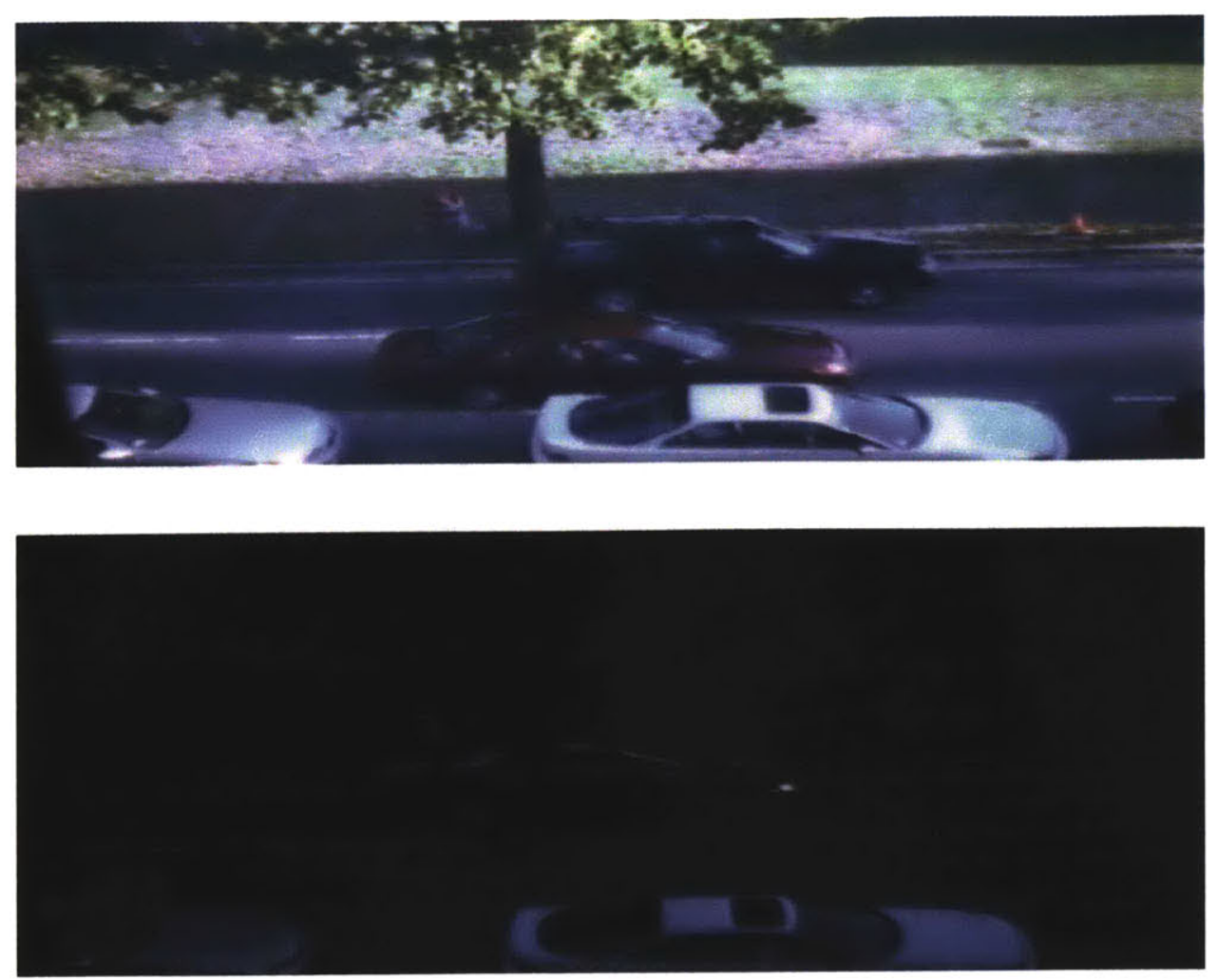

Figure 4-23: Sample of Lighting Shift

shows an example of the lighting shift between images in set 2 . With the darker scene, there are fewer edges in the images, resulting in a larger compression ratio. The lower compression ratios in image sets 4 and 5 are due to the large number of edges in the images. 


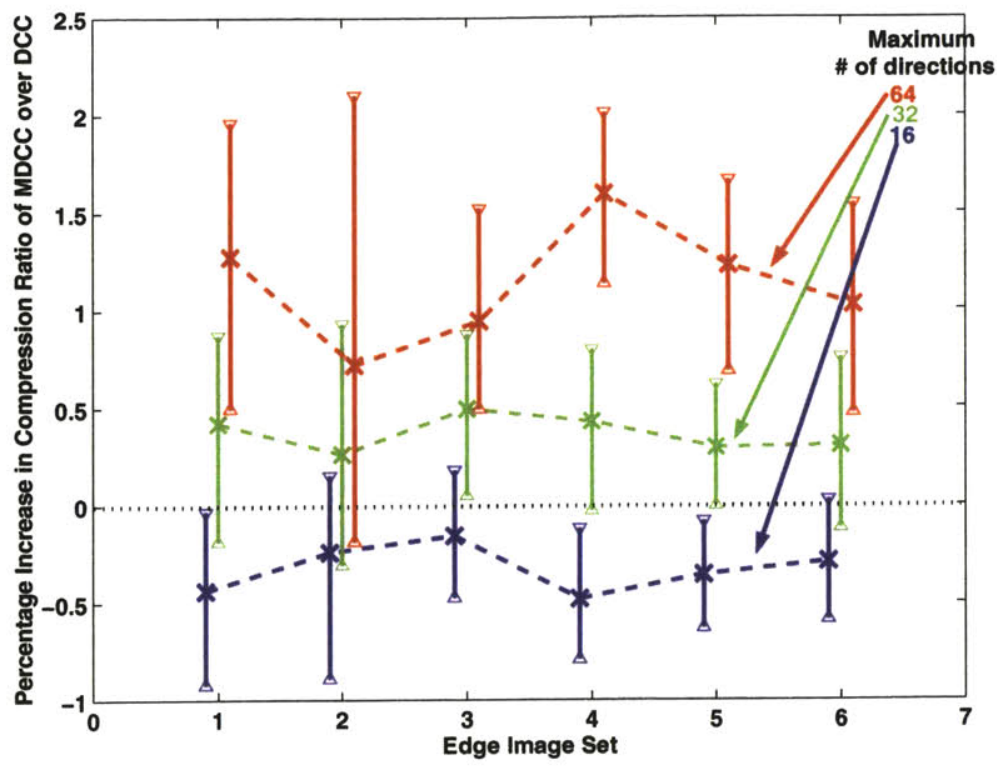

Figure 4-24: Range of Percentage Increase in Compression Ratio of MDCC over DCC for edge images with maximum number of direction codes 16, 32, and 64 .

To quantify the comparison of DCC with MDCC, we look at the percentage increase in compression ratio of MDCC over DCC $\left(\frac{\left(C_{M D C C}-C_{D C C}\right)}{C_{D C C}} * 100\right)$. Figure 4-24 shows the average and range of the percentage increase in compression ratio for edge images. The dotted line represents no increase in compression ratio, where MDCC and DCC give the same results. The average for each image set is marked and the averages for the same maximum number of directions are connected with a dashed line. The range for each average is represented by the vertical lines. There is a slight horizontal shift of the averages for each maximum number of directions for easier viewing.

We see that with 16 as the maximum number of directions there is a decrease in compression ratio from DCC to MDCC. This decrease is caused by incomplete coding of splits in the images as described in section 4.1.3. Therefore, the average number of directions in the contour codes is larger than 16 . With 32 and 64 , there is on average 
an increase in compression ratio of MDCC over DCC for the edge images. As the maximum number of directions increases, the benefits of MDCC increase.

The maximum number of direction codes significantly changes the performance of MDCC. MDCC benefits from traversing the entire contour, reducing the number of contours (start locations) to code for an image. There is a tradeoff between the maximum number of direction codes and the compression ratio. The optimal maximum number of direction codes varies with each image. Finding the optimal compression ratio for each image is not practical. At the image sensor, the average number of directions can be monitored to periodically change the maximum number of direction codes to improve compression.

Compression ratios of full color images are higher than edge images. Full color images are 24 bits per pixel (bpp), while edge images are $1 \mathrm{bpp}$. In the case of MDCC with maximum number of direction codes of 64 , the compression of edge images required $0.04-0.6 \mathrm{bpp}$. The compressed full color images include both color and distance information. This additional information raises the compression to $0.3-1.2 \mathrm{bpp}$. The compression ratios for full color images with maximum number of direction codes equal to 64 are shown in Figure 4-25. In full color images, fewer contours will reach the maximum number of direction codes. The additional requirement that the distance of the edges are similar over the entire contour, reduces the number of edges in a contour. MDCC performance improves when contours do not exceed the maximum number of direction codes, resulting in a more visible increase in the compression ratio over $\mathrm{DCC}$.

As in the case of edge images, we present the average and range of percentage 


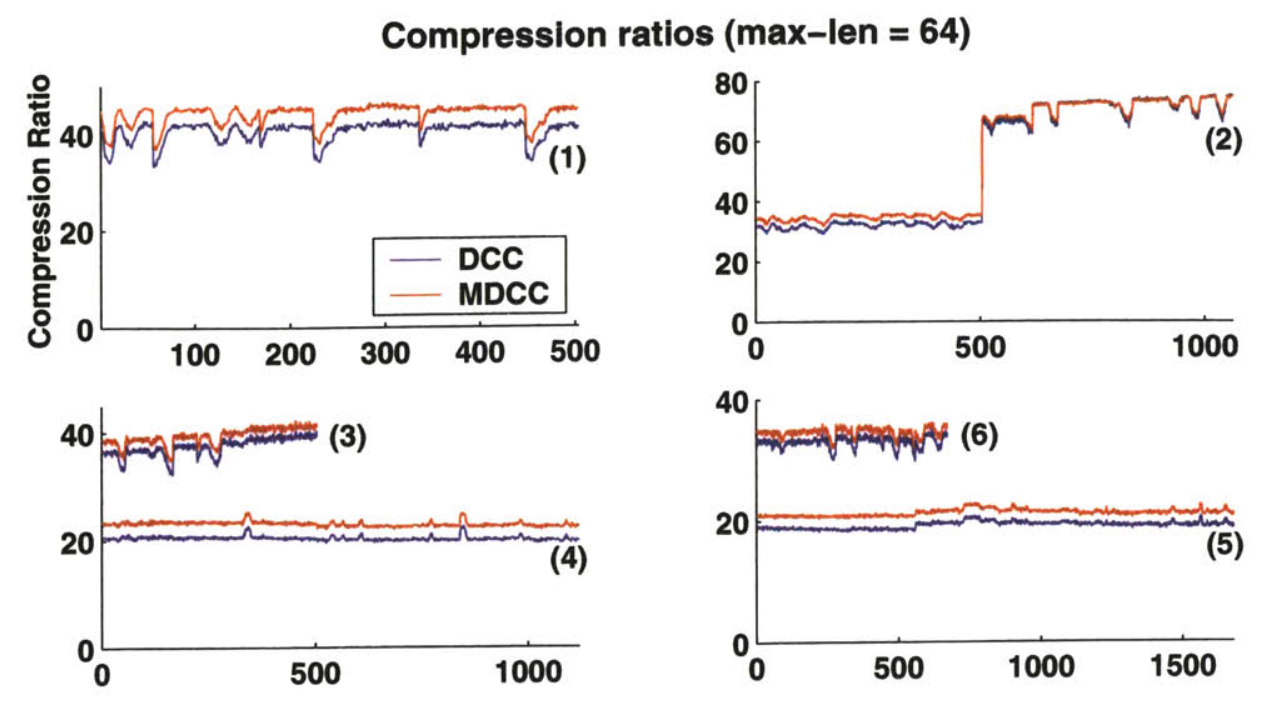

Figure 4-25: Compression Ratio of Images with maximum direction codes of 64

increase in compression ratio of MDCC over DCC for full color images in Figure 426. Image sets $1,3,4$, and 5 show an average increase with maximum number of

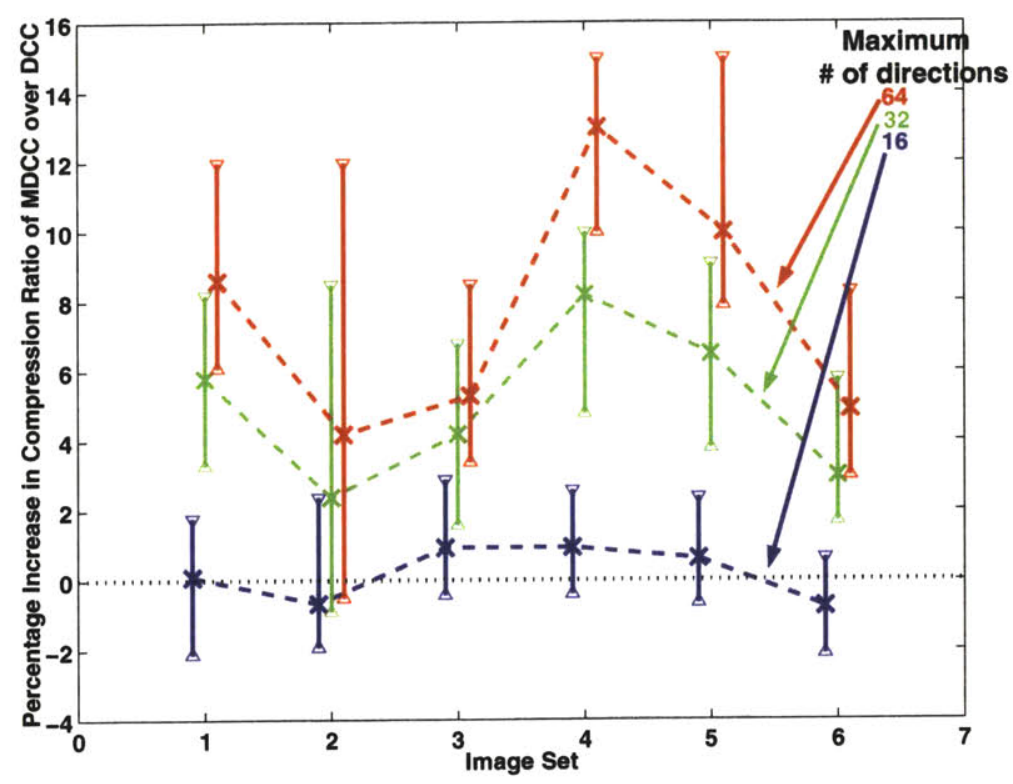

Figure 4-26: Range of Percentage Increase in Compression Ratio of MDCC over DCC for full color images with maximum number of direction codes 16, 32, and 64 .

direction codes equal to 16 , demonstrating the benefit of a reduced number edges per contour. There continues to be better performance in MDCC with the increase in the maximum number of directions. 
The compression method presented in this chapter is a contour based method, that is content dependent. The number of edges in the images significantly effects the compression ratio. The fewer the edges, the higher the compression will be. With the modifications to DCC to include splits in the contour code (MDCC), an improvement in compression performance can be realized (particularly for full color images). This improvement is dependent upon the maximum number of directions allowed in a contour. The maximum number of directions must be large enough to complete the coding of contours with splits, but small enough not to significantly add bits to the contours without splits. With the appropriate maximum number of directions, MDCC can be used instead of DCC in the compression method to increase compression of the images. 


\section{Chapter 5}

\section{Stereo Vision}

The compression method incorporates depth information to enhance processing of the compressed image data. We use images from two cameras to obtain depth information, this process is known as stereo vision. The cameras are located in different positions while images are captured simultaneously. Corresponding points from the images are used to estimate depth. In order to calculate the depth, the relative and interior orientation of the cameras must be determined [35]. The relative orientation is the transformation from one camera coordinate system to the other, characterized by a rotation and translation. The interior orientation is the transformation from camera coordinates (3d) to image coordinates (2d). We can align the camera and image coordinate systems to remove rotation, but the location of the origin (principal point), distance from the lens to the image plane (principal distance), pixel size and aspect ratio must still be found.

In order to estimate the depth, the cameras must be calibrated and corresponding points must be found. Calibration of the cameras refers to the process of obtaining 
the cameras' parameters (interior and relative orientation of the cameras) from two images. Calibration is accomplished by solving a system of nonlinear equations. Image measurements are inaccurate, so least squares methods are used. This chapter begins with a description of the cameras' calibration.

It is also necessary to find pairs of corresponding points in the two images to determine the depth. As a component of the compression algorithm depth information is only needed for the contours, therefore we only estimate the depth of edges. Corresponding points are found using normalized correlation. In this chapter, we present the stereo vision algorithm used in the system. The chapter focuses on calibration (relative and interior orientation) and finding correspondences of image points.

\subsection{Calibration}

Calibration is the process of determining the cameras' parameters from image measurements [35]. Calibration is normally accomplished by taking images of a specifically designed target or calibration object of known size and distance from the cameras. A typical calibration object consists of a regular grid of points, lines or area where the geometry is known. The correspondences of points are labeled and a least squares method is used to determine the camera parameters. Due to the setup of our system, this method of calibration was not practical. Because of the large baseline $(0.5 \mathrm{~m})$, the setup was dismantled during transportation. The position of the cameras outdoors and proximity to traffic made calibration difficult. To calibrate the cameras we manually identify corresponding points and used an iterative least squares method 
to estimate the cameras' parameters. Because the absolute scale of the scene is not known, the resulting depths differ from the true depths by a scale factor. We calibrate the cameras for each day (image set). This section describes the determination of relative orientation and gives the estimated cameras' parameters for each day.

\subsubsection{Camera Model}

A pinhole camera model is a close approximation of the projection in a camera. Light is projected onto an image plane through the optical center of the camera as shown in Figure 5-1. The perpendicular distance from the optical center to the image plane is the principal distance. The principal point is the projection of the optical center on to the image plane. The optical axis is normal to the image plane. There are two coordinate systems of interest: 1 ) camera coordinates (3D) where the origin is at the optical center of the camera, and 2) image coordinates (2D) with the origin at the principal point. In Figure $5-1$ the center of projection is at $(0,0,0)$ in camera coordinates and the optical axis is in the $Z$-direction. $P=(X, Y, Z)^{T}$ is a point on the object in camera coordinates, and $p=(x, y)^{T}$ is the corresponding point from the image of the object. The principal point is $(0,0, f)$ in camera coordinates. From similar triangles $O p q$ and $O P Q$,

$$
\frac{x}{f}=\frac{X}{Z} \text { and } \frac{y}{f}=\frac{Y}{Z}
$$




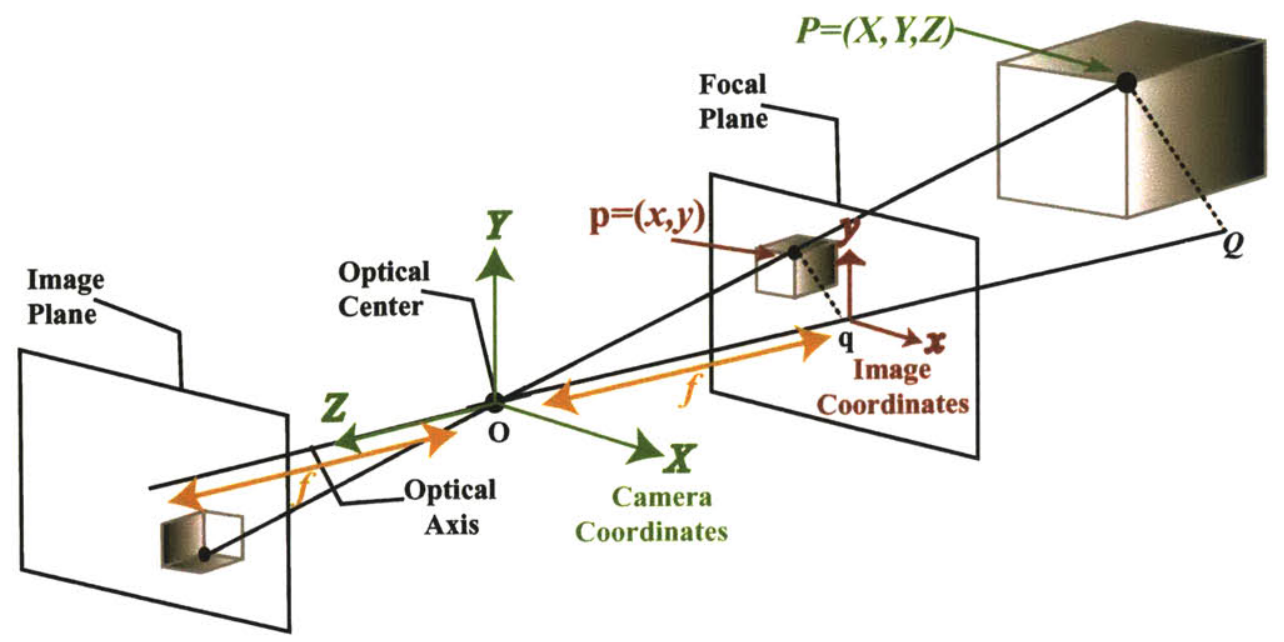

Figure 5-1: Pinhole Camera Model

\subsubsection{Epipolar Constraint}

In a stereo vision system, two cameras are positioned in two different locations. The $3 \mathrm{D}$ position of an imaged point is determined from corresponding points in the two image planes. Given a point in one image, there is a geometric constraint that limits the possible locations of the corresponding point in the second image, this is called the epipolar constraint. The corresponding point is restricted to the line formed by the intersection of the image plane and the plane created by the object point and the cameras' optical centers. This plane is called the epipolar plane. The epipolar plane in Figure 5-2 is the triangle with vertices $P, O_{l}$, and $O_{r}$. The line where the epipolar plane intersects the image plane is the epipolar line and the location of the corresponding point is restricted to this line. The position of the corresponding point on the epipolar line determines the distance to the object point in three dimensions. 


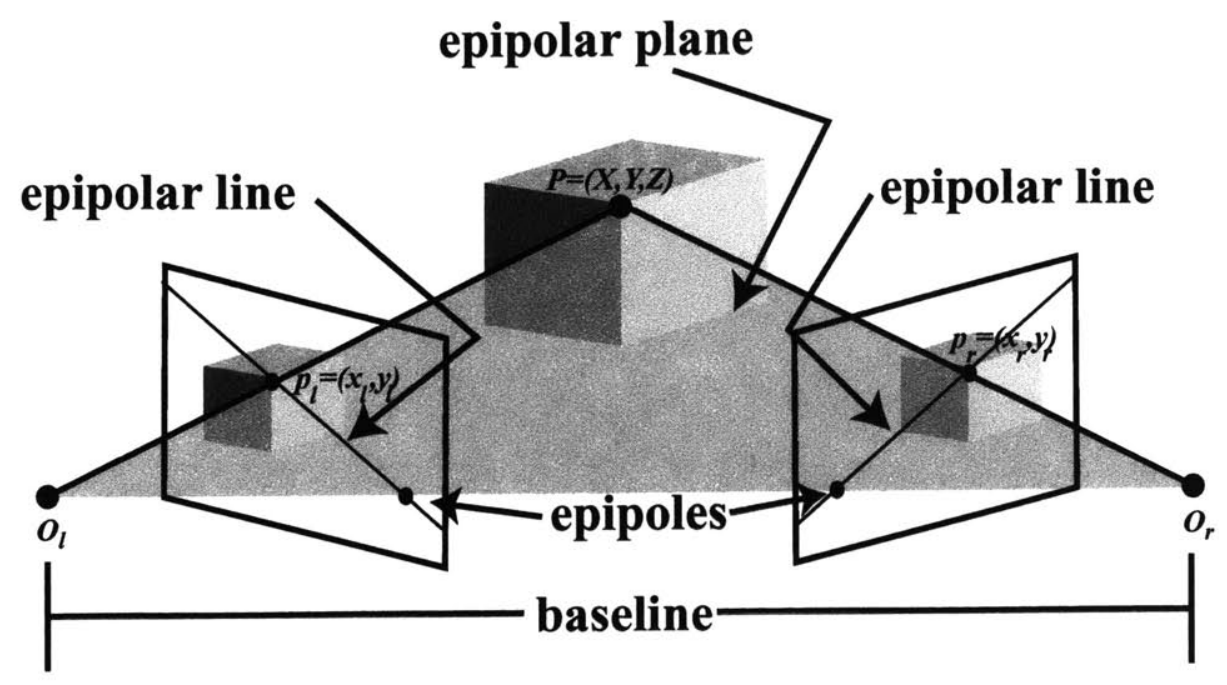

Figure 5-2: Epipolar Constraint

\subsubsection{Relative Orientation}

The location of the object can be determined given corresponding points in the image plane of the left and right camera coordinates and the relative orientation of the two cameras. The relative orientation is described by the rotation and translation of the coordinate system of one camera relative to that of the other. Starting from the left camera coordinate system and going to the right, we have

$$
\mathbf{R}\left[\begin{array}{c}
X_{l} \\
Y_{l} \\
Z_{l}
\end{array}\right]+\mathbf{b}=\left[\begin{array}{c}
X_{r} \\
Y_{r} \\
Z_{r}
\end{array}\right]
$$

where $\left(X_{l}, Y_{l}, Z_{l}\right)^{T}$ and $\left(X_{r}, Y_{r}, Z_{r}\right)^{T}$ are the coordinates of an object point in the left and right camera coordinate systems, $\mathbf{R}$ is the rotation matrix and $\mathbf{b}$ is the translation vector (baseline) from the left camera coordinate to the right camera coordinate. Given corresponding image points $\left(x_{l}, y_{l}\right)$ and $\left(x_{r}, y_{r}\right)$, from the left and right image, 
the depth, $Z_{l}$ and $Z_{r}$ with respect to the left and right camera respectively, can be calculated. We see by solving for $X$ and $Y$ in (5.1) and substituting $X$ and $Y$ into (5.2) for the left and right cameras, that we have

$$
\begin{gathered}
\mathbf{R}\left[\begin{array}{c}
\frac{Z_{l} x_{l}}{f_{l}} \\
\frac{Z_{l} y_{l}}{f_{l}} \\
Z_{l}
\end{array}\right]+\mathbf{b}=\left[\begin{array}{c}
\frac{Z_{r} x_{r}}{f_{r}} \\
\frac{Z_{r} y_{r}}{f_{r}} \\
Z_{r}
\end{array}\right] \\
\mathbf{R}\left[\begin{array}{c}
\frac{x_{l}}{f_{l}} \\
\frac{y_{l}}{f_{l}} \\
1
\end{array}\right] Z_{l}+\mathbf{b}=\left[\begin{array}{c}
\frac{x_{r}}{f_{r}} \\
\frac{y_{r}}{f_{r}} \\
1
\end{array}\right] Z_{r}
\end{gathered}
$$

We can solve for $Z_{l}$ and $Z_{r}$, depth information, using any two equations from (5.3) (refer to Horn [35] pp. 311-312 for a more detailed discussion).

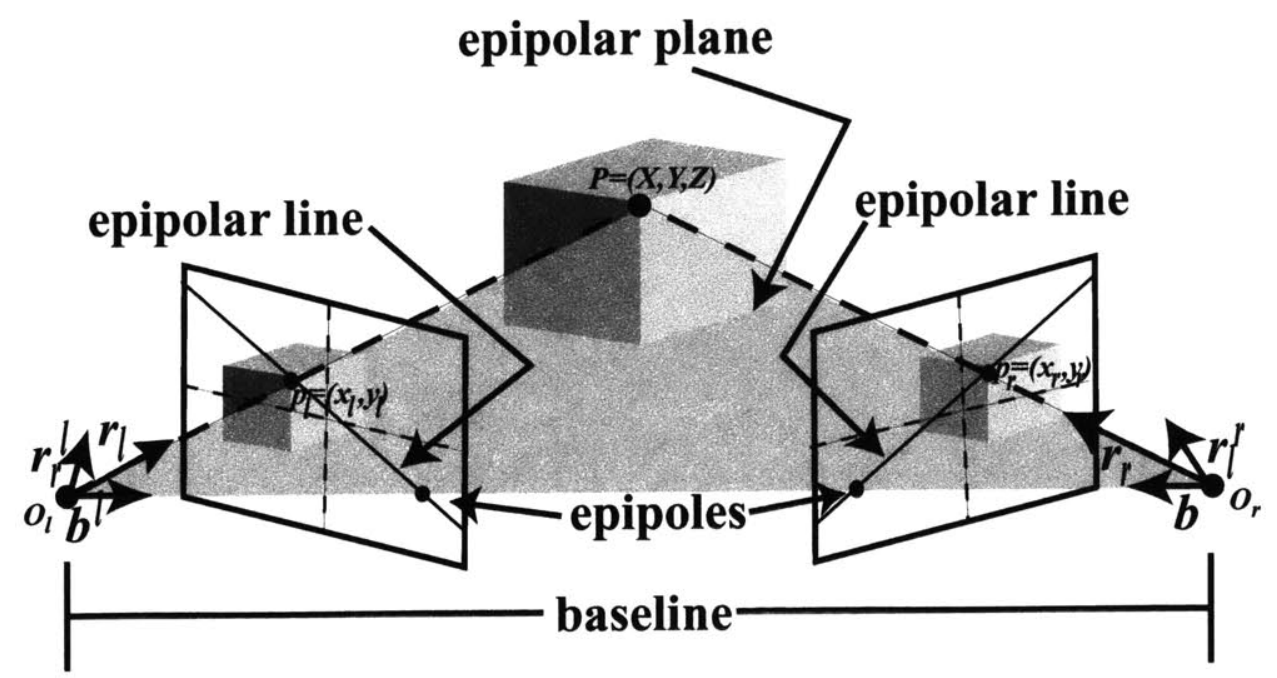

Figure 5-3: Relative Orientation

In order to calculate the depth we need the interior orientation, the relative orientation and corresponding image points. The relative orientation is characterized by 
the rotation and translation from one camera coordinate to the other. Horn $[35,36,37]$ showed that the relative orientation can be recovered with five or more corresponding image points. Let $\mathbf{r}_{r}=\left(x_{r}, y_{r}, f_{r}\right)^{T}$ be the vector from the right optical center to an image point in the right camera coordinate system, and let $\mathbf{r}_{l}=\left(x_{l}, y_{l}, f_{l}\right)^{T}$ be the vector from the left optical center to the corresponding image point in the left camera coordinate system. Then $\mathbf{r}_{l}$ can be rotated into the right coordinate system, where $\mathbf{r}_{l}^{r}=\mathbf{R r}_{l}$. From (5.3), $\mathbf{r}_{r}=\mathbf{r}_{l}^{r} \frac{Z_{l}}{f_{l}}+\mathbf{b}$, therefore $\mathbf{r}_{r}, \mathbf{b}$, and $\mathbf{r}_{l}^{r}$ are coplanar (hence $\left.\left[\begin{array}{lll}\mathbf{b} & \mathbf{r}_{r} & \mathbf{r}_{l}^{r}\end{array}\right]=0\right)$. A scaled version of the vectors is shown in Figure 5-3.

\section{Finding Cameras' Parameters}

The cameras' parameters are found in three steps: 1) manually identifying corresponding points, 2) initialize cameras' parameters, and 3) use non-linear least squares method to minimize the error. Corresponding points are found manually in sample images, Figure 5-4 is one example of a set of point correspondences found, where for example the points marked by 42 (lower left of both images) in each image are corresponding points.

The cameras' parameters consist of $\mathbf{R}, \mathbf{b}, f_{l}, f_{r}$, and the principal points of each camera. The cameras were placed on a metal bar to approximately align the optical axes of the cameras, so a good initial guess for $\mathbf{R}$ is the identity matrix and a good initial guess for $\mathbf{b}$ is the vector $(1,0,0)$. The principal distances are initialized to the specified lens focal length, in our case $25 \mathrm{~mm}$, and the principal points are assumed to be at the centers of the images. The non-linear least squares method for relative orientation uses these parameters to start an iterative procedure that minimizes an 

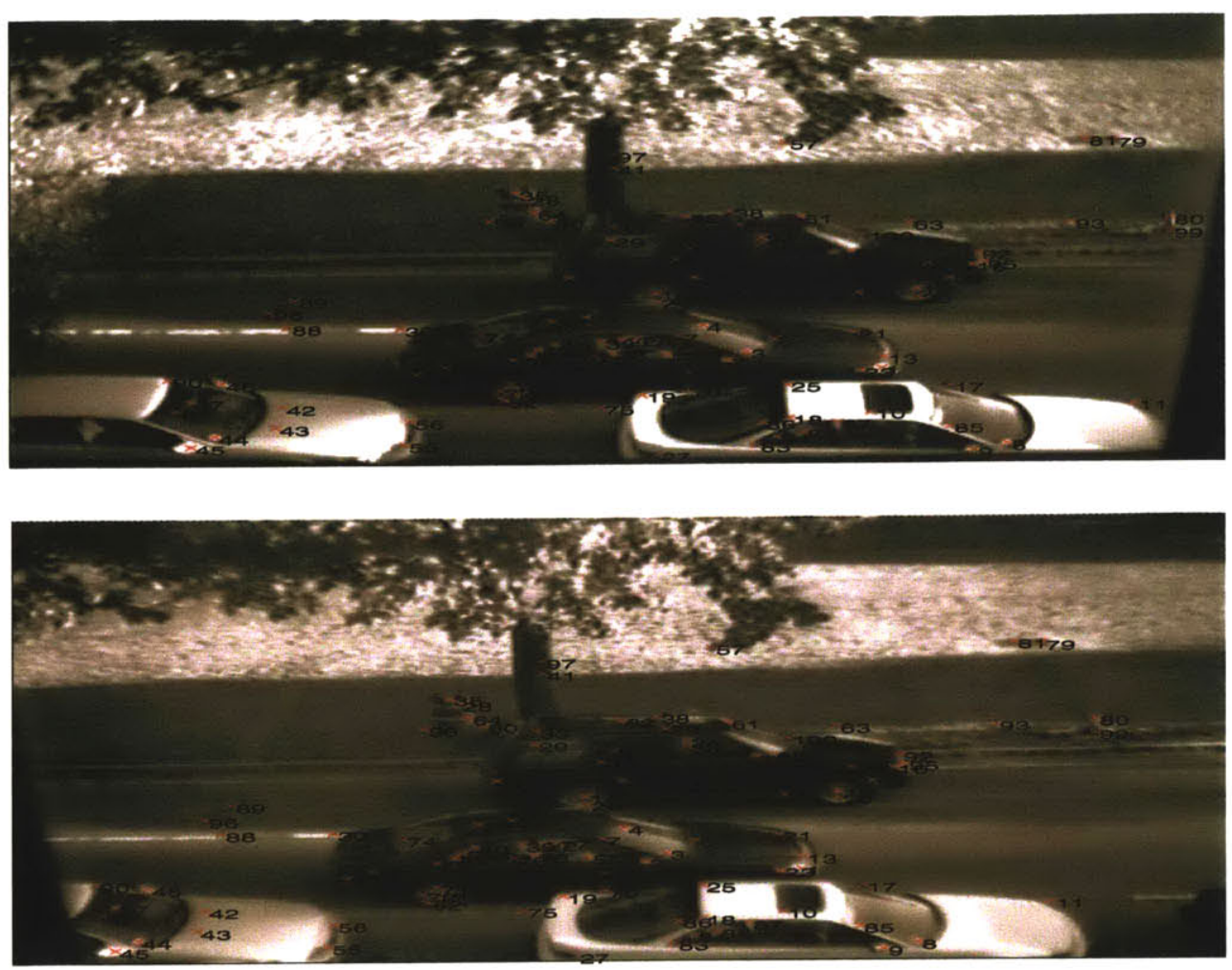

Figure 5-4: Manual Correspondence

error function within a set of constraints.

The epipolar constraint requires that the point in one image corresponding to a point in the other image lie on a particular epipolar line. A measure of error is the distance of the corresponding point from the epipolar line calculated with the cameras' parameters. The total error, $e$ is the sum of $e_{r, i}$, the distance in the image plane from the image of $\mathbf{r}_{l, i}^{r}$ to the right epipolar line and $e_{l, i}$, the distance in the image plane from the image of $\mathbf{r}_{r, i}^{l}$ to the epipolar line, where $\mathbf{r}_{r, i}^{l}$ is the vector from the right optical center to the image point $i$ in the right image rotated to align the right coordinate with the left coordinate system, $\left(\mathbf{r}_{r}^{l}=\mathbf{R}^{-1} \mathbf{r}_{r}\right)$. The errors, $e_{r, i}$ and 
$e_{l, i}$, are

$$
\begin{aligned}
& e_{r, i}=\frac{\left[\mathbf{b} \mathbf{r}_{r, i} \mathbf{r}_{l, i}^{r}\right]\left|\left(\mathbf{b} \times \mathbf{r}_{r, i}\right)_{x, y}\right|}{\left|\mathbf{b} \times \mathbf{r}_{r, i}\right|} \\
& e_{l, i}=\frac{\left[\mathbf{b}^{l} \mathbf{r}_{r, i}^{l} \mathbf{r}_{l, i}\right]\left|\left(\mathbf{b}^{l} \times \mathbf{r}_{r, i}^{l}\right)_{x, y}\right|}{\left|\mathbf{b}^{l} \times \mathbf{r}_{r, i}^{l}\right|}
\end{aligned}
$$

where $\mathbf{b}^{l}=-\mathbf{R}^{-1} \mathbf{b}$ is the translation in the left camera coordinates and $\mid\left(\mathbf{b}^{l} \times\right.$ $\left.\mathbf{r}_{r, i}^{l}\right)_{x, y} \mid$ is the magnitude of the cross product with $x$ and $y$ components only (this is the area of the parallelogram projected onto the image plane). To estimate the cameras' parameters, we use Levenberg-Marqurdt least square optimization method implemented in MINPACK function LMDIF to minimize

$$
e^{2}=\sum_{i=1}^{N}\left(e_{l, i}^{2}+e_{r, i}^{2}\right)
$$

where $N$ is the number of image point pairs used for calibration. Figure 5-5 is a plot of the average error per point pair,

$$
\hat{e}=\sqrt{\frac{1}{2 N} e^{2}}
$$

for each image set.

\subsection{Our Method}

We process only vertical edge features, this increases the spread of the stereo vision algorithm. We begin with finding vertical edges to subpixel accuracy for both im- 


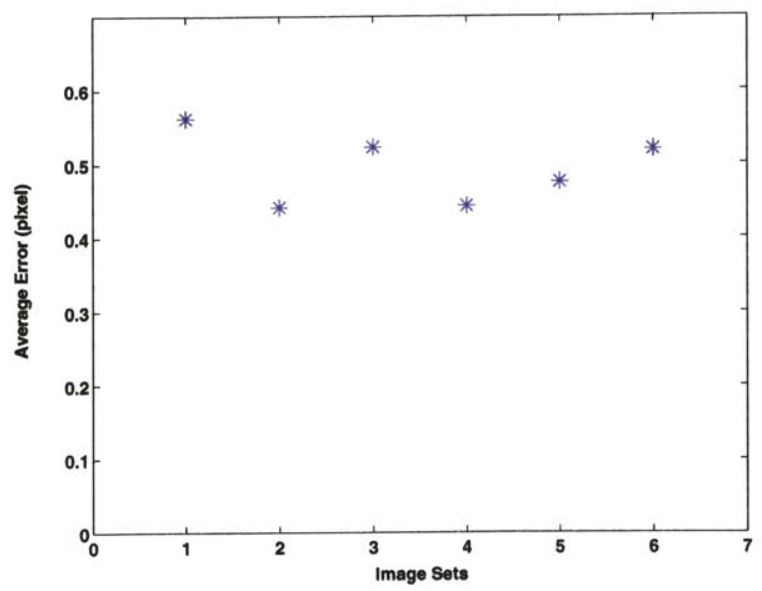

Figure 5-5: Average error per point pair from corresponding point to epipolar line ages. A point from the left image determines the epipolar line to search in the right image. Normalized correlation is used to find the best match and depth is calculated. Figure 5-6 is a flow chart of the algorithm. This section describes the stereo vision algorithm.

\subsubsection{Preprocessing}

Preprocessing is performed to enhance some detail, eliminate irrelevant information and normalize the images. Our preprocessing consists simply of finding the vertical edges to sub-pixel accuracy. Since the cameras are roughly horizontally aligned, the epipolar lines will be horizontal. Using horizontal edges will generate many false correspondences. A corresponding point on a horizontal edge line will be difficult to find. The epipolar line and the horizontal edge line will be coincident producing a large number of possible correspondences. The large number of possible correspondences increase the likelihood of finding false correspondences. We use only vertical edges, to reduce the number of false correspondences, because there will be a small number of 


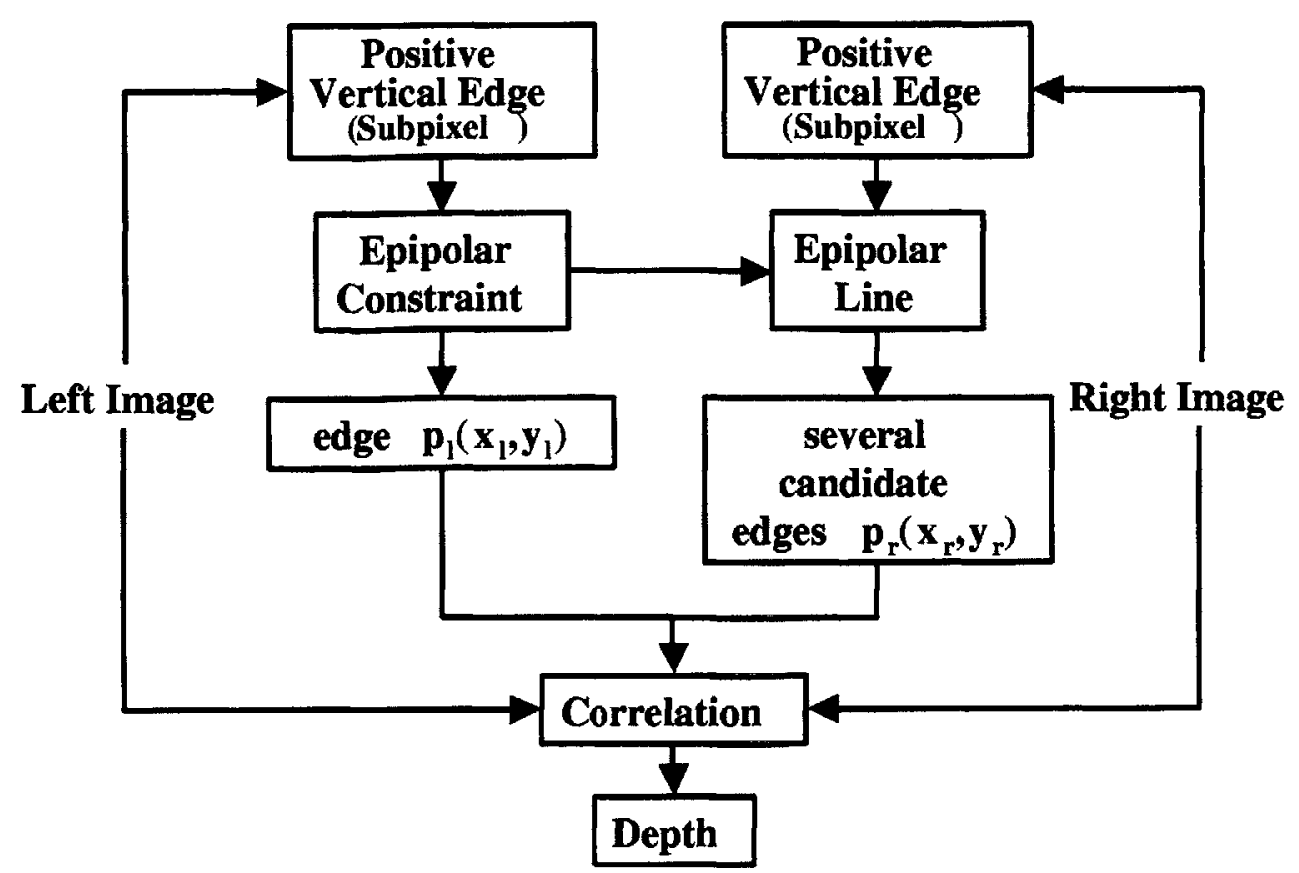

Figure 5-6: Stereo Vision Flow chart

possible correspondences. The remainder of this section describes the edge detection method chosen and the calculation to sub-pixel accuracy.

\section{Edge Detection}

Edge detection consists of finding local maxima in the gradient. This system uses a Sobel operator. The Sobel edge detection method was decided upon simply based on speed. Only the vertical edges are used in the distance calculation. The intensity gradient in the $x$-direction is estimated using the difference operator

$$
\begin{aligned}
G_{x}(i, j)= & I(i+1, j-1)+2 I(i+1, j)+I(i+1, j+1) \\
& -(I(i-1, j-1)+2 I(i-1, j)+I(i-1, j+1)) .
\end{aligned}
$$

This kernel is shown in Figure 5-7. The positive and negative edges are distinguished 


\begin{tabular}{|l|l|l|}
\hline-1 & 0 & 1 \\
\hline-2 & 0 & 2 \\
\hline-1 & 0 & 1 \\
\hline
\end{tabular}

Figure 5-7: Sobel Kernel

to reduce the possibility of false correspondence. Figure 5-8 and 5-9 show examples of the positive and negative vertical edges of the original images. The left and right edge images are located with sub-pixel accuracy, using an interpolation method.

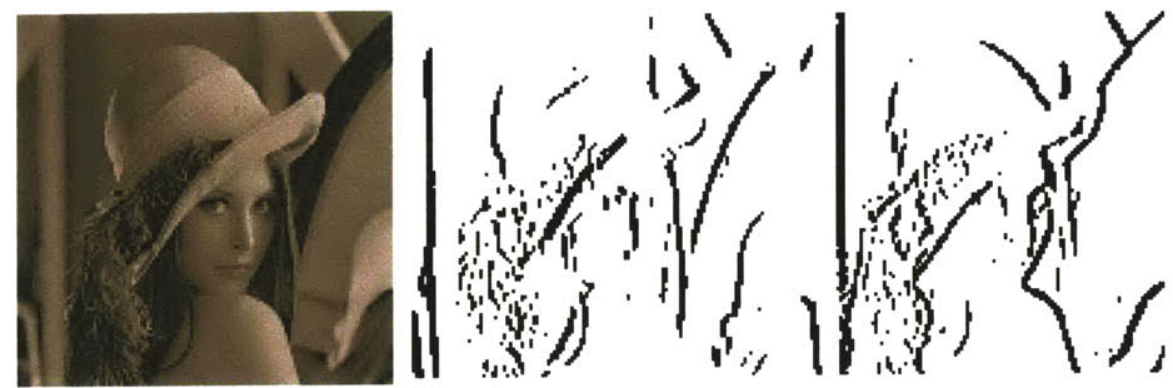

Figure 5-8: Original image, positive and negative vertical edges

\section{Sub-pixel Accuracy}

Sub-pixel accuracy is used to greatly expand the effective resolution of the image through interpolation. The "true" edge may occur anywhere between two pixels. This system uses a parabolic fit to estimate the position of the edge. A parabolic fit is computationally cheap and provides a good estimate of the edge location. The gradient values at three pixels are used to approximate a parabola. The location of the maximum of the parabola, $x_{\max }$, can be determined within a fraction of a pixel, thus the term sub-pixel accuracy. Figure 5-10 shows the parabolic approximation, and the calculated maximum. Let $G_{0}, G_{1}$, and $G_{2}$ be the magnitudes of the three horizontally adjacent gradients (where $G_{1}$ is a local maxima) and $x_{0}=0, x_{1}=1$, and $x_{2}=2$ be the corresponding horizontal coordinates. Using the equation for a 


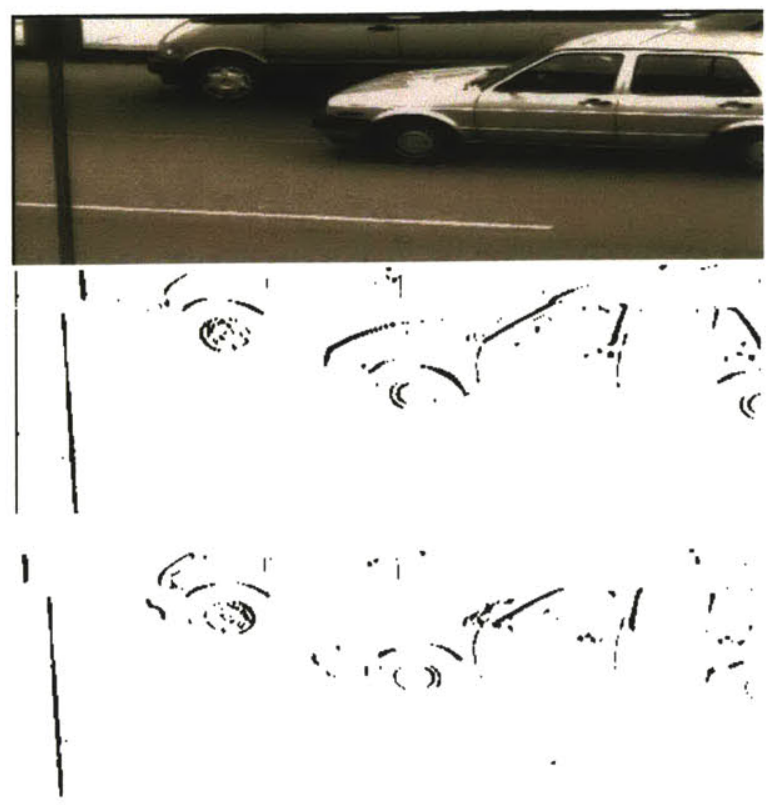

Figure 5-9: Additional Vertical Edges Example

parabola, $G=a x^{2}+b x+c$, the maximum occurs when $\frac{d G}{d x}=2 a x+b=0$, therefore $x_{\max }=-\frac{b}{2 a} \cdot x_{\max }$ is approximated by solving for $a$ and $b$ from the three adjacent gradients.

$$
\begin{aligned}
G_{0} & =c, \\
G_{1} & =a+b+c, \text { and } \\
G_{2} & =4 a+2 b+c .
\end{aligned}
$$

Substituting for $c$ in $G_{1}$ and $G_{2}$, yields

$$
\begin{aligned}
& G_{1}-G_{0}=a+b \text { and } \\
& G_{2}-G_{0}=4 a+2 b,
\end{aligned}
$$

then solving for $a$ and $b$

$$
\begin{aligned}
& a=-G_{1}+0.5 G_{0}+0.5 G_{2} \\
& b=2 G_{1}-1.5 G_{0}-0.5 G_{2} .
\end{aligned}
$$

The equation for the maximum is

$$
x_{\max }=\frac{G_{1}-0.75 G_{0}-0.25 G_{2}}{G_{1}-0.5 G_{0}-0.5 G_{2}}+x_{0} .
$$




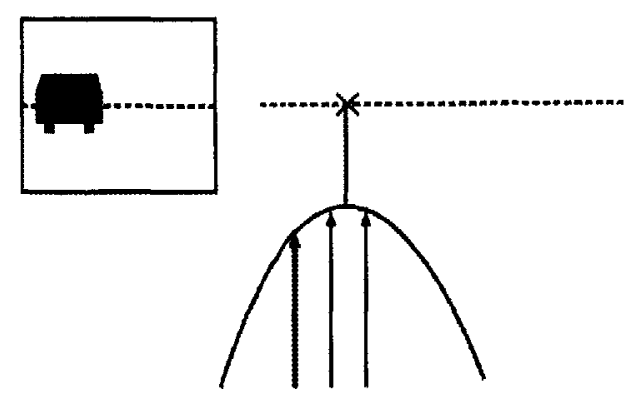

Figure 5-10: Subpixel Approximation

\subsubsection{Depth}

In order to determine the depth, an object point must be imaged by both cameras. Finding corresponding points in the two images is a matching problem. With differences in camera position, viewing direction, and camera properties, finding the match in two different images can be difficult. Brightness differences in the images result from the different viewpoints of the cameras as well as the aperture settings and sensor responses of the two cameras. Aside from brightness differences, partial occlusion may interfere in one camera's view and not the other, making it hard to find correspondences. Finding correspondence accurately and fast is a difficult problem to solve.

We use the epipolar constraint to greatly reduce the search region. Given an edge point in the right image, the corresponding epipolar line in the left image is computed. The corresponding point is determined by giving a score to each edge point on the epipolar line. The point with the largest score, above a threshold, is considered to be the corresponding point. Only edge points of matching polarity are considered for matching. 
The score is a normalized correlation[75]. The $(2 n+1) \times(2 m+1)$ area centered at a point, $p_{1}=\left(u_{1}, v_{1}\right)$, in the right image is correlated with the $(2 n+1) \times(2 m+1)$ area centered at a point, $p_{2}=\left(u_{2}, v_{2}\right)$, in the left image. The score is

$$
c\left(p_{1}, p_{2}\right)=\frac{\sum_{i=-n}^{n} \sum_{j=-m}^{m}\left[I_{1}\left(u_{1}+i, v_{1}+j\right)-\overline{I_{1}\left(u_{1}, v_{1}\right)}\right]\left[I_{2}\left(u_{2}+i, v_{2}+j\right)-\overline{I_{2}\left(u_{2}, v_{2}\right)}\right]}{(2 n+1)(2 m+1) \sigma\left(I_{1}\left(u_{1}, v_{1}\right)\right) \sigma\left(I_{2}\left(u_{2}, v_{2}\right)\right)}
$$

where $I_{k}(u, v)$ is the intensity at point $(u, v)$ of image $k$, and

$$
\overline{I_{k}(u, v)}=\frac{\sum_{i=-n}^{n} \sum_{j=-m}^{m} I_{k}(u+i, v+j)}{(2 m+1)(2 n+1)}
$$

is the average intensity, and

$$
\sigma\left(I_{k}(u, v)\right)=\sqrt{\frac{\sum_{i=-n}^{n} \sum_{j=-m}^{m} I_{k}^{2}(u+i, v+j)}{(2 m+1)(2 n+1)}-{\overline{I_{k}(u, v)}}^{2}}
$$

is the standard deviation of the area centered at $(u, v)$ for image $k$. The normalized correlation has a maximum of 1 if the areas are identical, and less if they are not (with a minimum possible score of -1 if the areas are complementary). Figure $5-11$ is an example of three correspondences found using the epipolar constraint and normalized correlation.

The depth of the edge pixel can be determined once the relative orientations are known and the correct correspondences are found. We can use two equations from equation 5.3 to find the depth of the pixel. Figure 5-12 displays several correspondence 

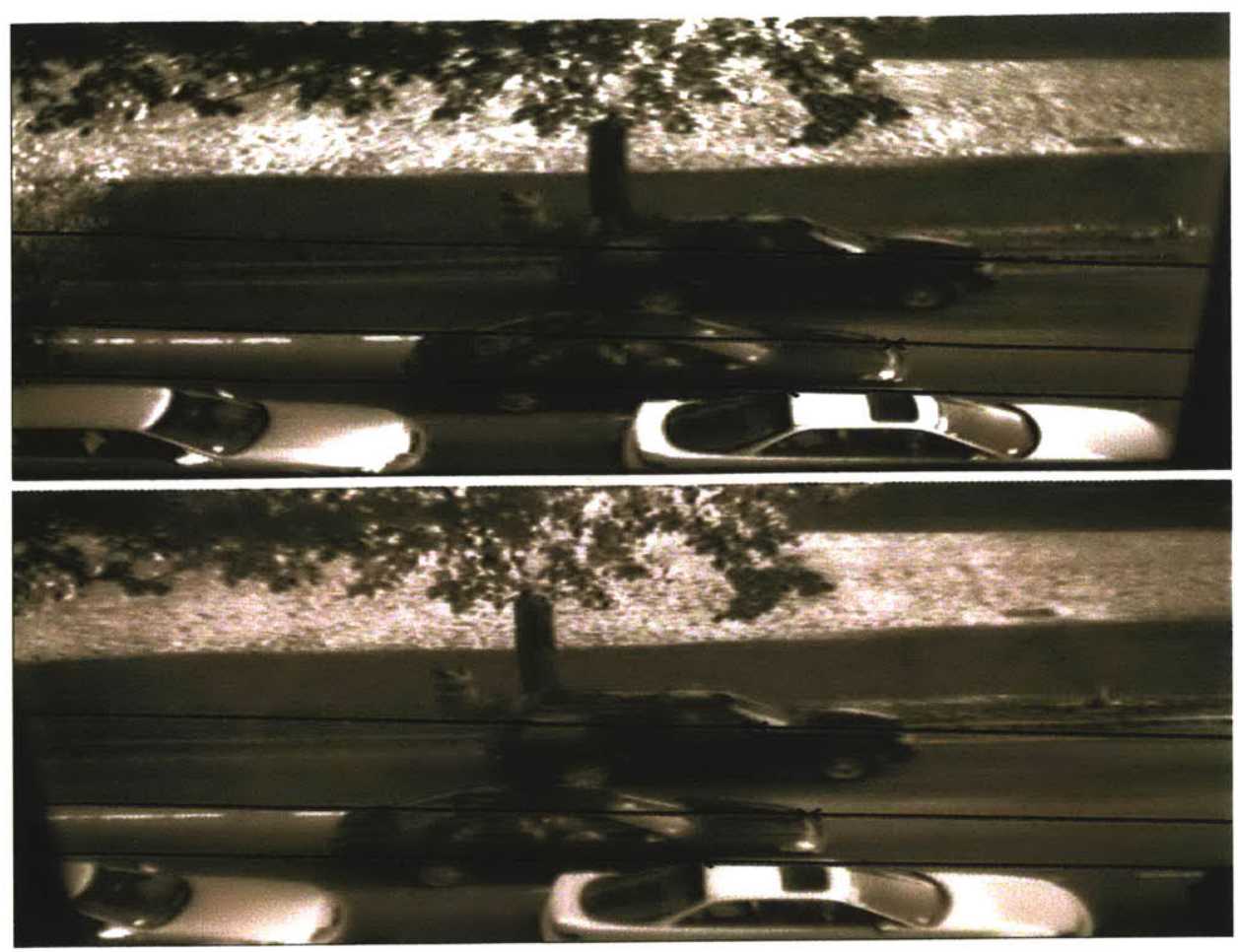

Figure 5-11: Correspondence using epipolar constraint and normalized correlation.

points and the associated depth.

Errors in correspondence occur primarily for two reasons, occlusion and coincidental local similarity of image patches corresponding to different objects. In Figure 5-12 occlusion is the cause of the false correspondence of points marked 22 in the center of both images. The region surrounding the corresponding points determined are similar to the correct corresponding points.

The depth information can be used to aid in distinguishing objects in the image. Depth of edge features in the image is added to the compressed image creating a 3D compressed image which is conducive to further processing. The depth and motion information from the compressed image data can be used to detect vehicles in the scene. 


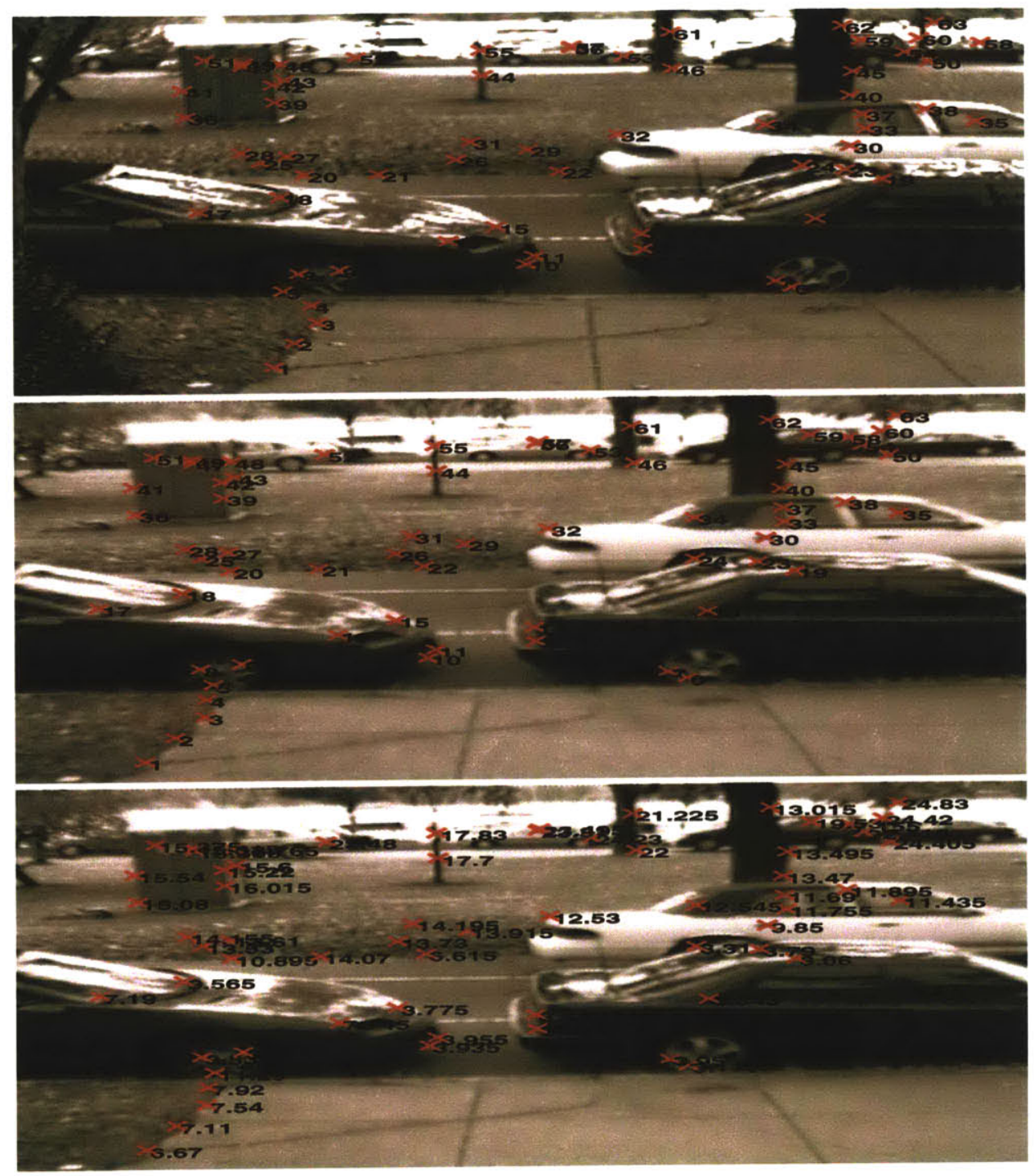

Figure 5-12: Sample of correspondences and depths 


\section{Chapter 6}

\section{Vehicle Detection and Tracking}

The purpose of the system is to provide traffic information to mobile agents. The image sensors find traffic information by detecting and tracking vehicles in sequences of compressed images data.

We assume that the camera view is limited to traffic lanes, therefore the main source of image change will be vehicle motion. With this assumption, we segment the images using motion vectors of the contours. The image of a vehicle consists of several contours. We use $k$-means clustering to cluster the contours into objects [16].

We focus on clusters that have non-zero motion and consider other clusters that appear not to move to be part of the background. This action removes parked vehicles, as well as trees, leaving mostly only moving vehicles. Tracking involves matching moving vehicles from one image to the next. This is accomplished by matching objects based on a set of features. This chapter presents the vehicle detection and tracking methods. 


\subsection{Vehicle Detection}

Traffic flow information is based on information derived from the detection of vehicles in a scene. Information (motion, color, edge, and depth) relevant to object detection is retained in the compressed image data. The contour's attributes retained by the compression algorithm can be used to detect the vehicles in the scene. For example, we classify contours as belonging to a particular object based on motion vectors using $k$-means clustering (see below). Each vehicle image is made up of several contours. The contours are grouped into objects, a bounding box is placed around the contours to specify the location of the object. The apparent objects' locations are continually modified based on the motion vectors from subsequent images.

\subsubsection{Feature Selection}

We classify contours into two groups, background and objects. In classification, the selection of feature is just as important as the particular classification method chosen. There are many possible features such as depth, color, motion vectors, size, and shape. Vehicle contours may have several colors from the body, windows, reflections, shadows, etc. Because of the different colors on the vehicles, color histograms may be useful in tracking vehicles, but not as helpful in object detection. We also initially considered classifying the contours based on depth alone. A histogram of the depth information from an image with one object of interest is shown in Figure 6-1. The figure displays three histograms of (i) background, (ii) object of interest (moving vehicle), and (iii) the entire image (both). There is no clear separation between 


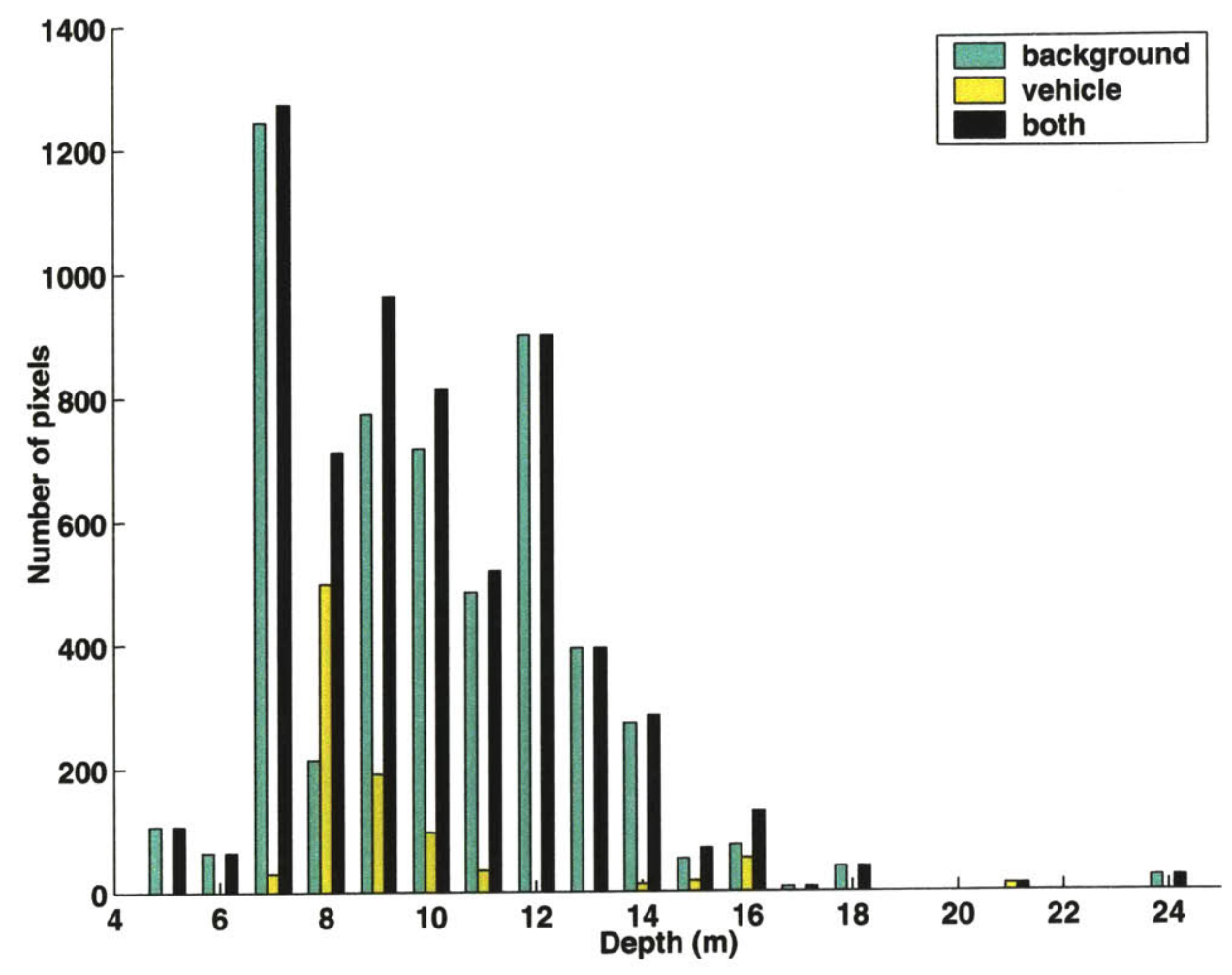

Figure 6-1: Histogram of Depth

the vehicle and the background. We next considered a combination of depth and of motion as a possible feature for object detection. In Figure 6-2, a horizontal line anywhere between 2-10 pixels does separate the vehicle from the background in the scatter plot of depth and motion vectors. This is why we chose to classify the contours based on the motion vector alone.

\subsection{2 $k$-Means Clustering}

Our objective in clustering the contours is to minimize the classification error, $e$. The average probability of error is

$$
P(e)=\int P(e \mid x) p(x) d x
$$




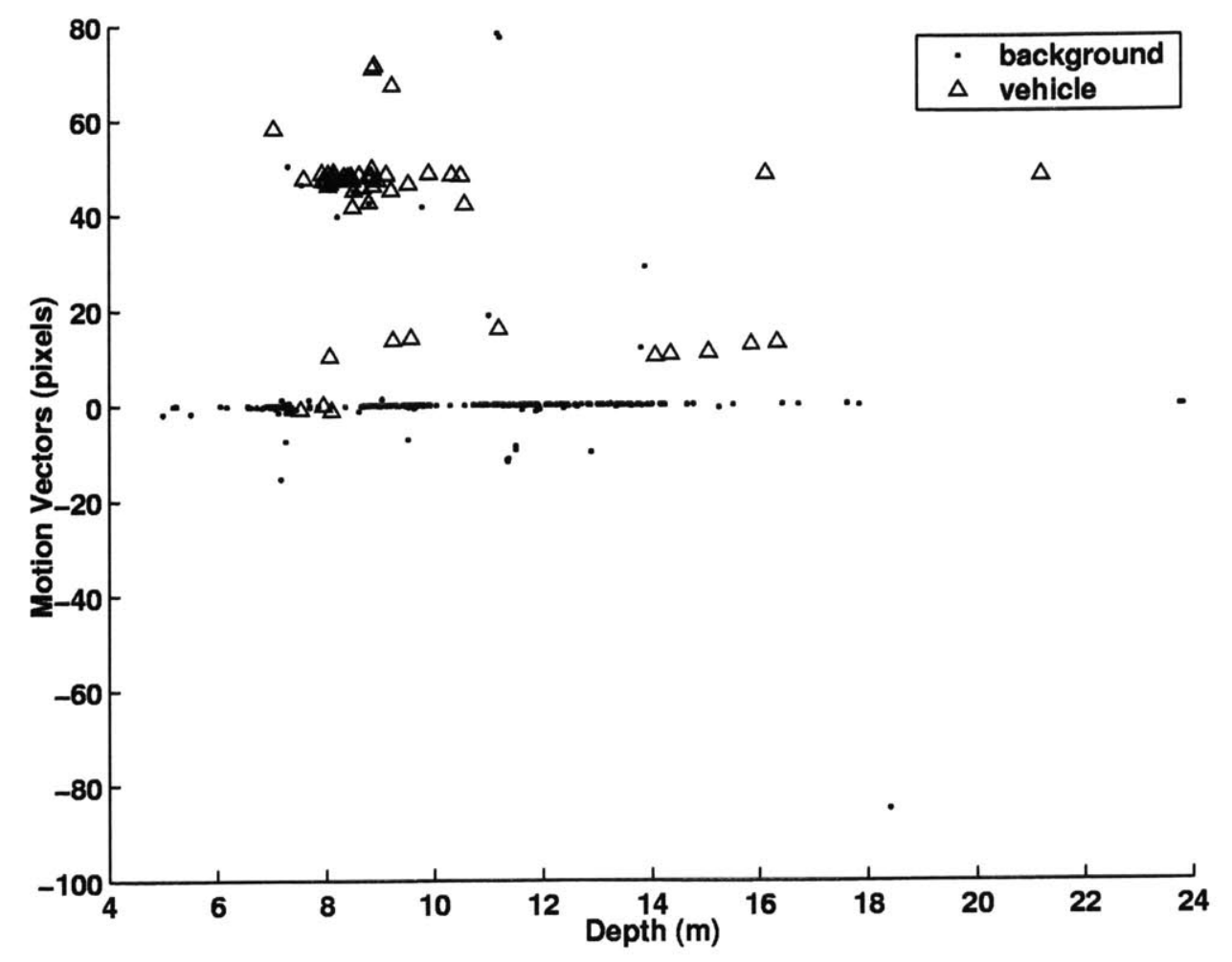

Figure 6-2: Scatter Plot of Motion Vectors vs. Depth

where $P(e \mid x)$ is the probability of error given a feature vector $x$, and $p(x)$ is the probability density of feature vector $x$. The probability of error given feature vector $x$ is $1-P\left(\omega_{j} \mid x\right)$, where $P\left(\omega_{j} \mid x\right)$, the posterior probability, is the probability of classification $\omega_{j}$ given feature vector $x$. In order to minimize the probability of error, we maximize the posterior probability $[15,16]$.

The feature vector $x$ is classified as being in cluster $j$, if $P\left(\omega_{j} \mid x\right)>P\left(\omega_{k} \mid x\right)$ for all $j \neq$ $k$. Given an estimate of the mean feature vector, $\mu_{j}$, for each state, we assume that the closer the feature vector is to the mean, $\mu_{j}$, the higher the posterior probability. This reduces the problem to finding the cluster means which minimize the sum of the square of the distance from the feature vectors to the mean. We use $k$-means clustering to estimate the cluster means and classify the feature vectors (see below). 
The iterative algorithm of $k$-means clustering estimates the cluster means, given $k$ initial guesses as described below. A feature vector can be in one of $k$ clusters. We determine the number of possible clusters using Dunn's index, a cluster validity measure described in the following section. We perform $k$-means clustering on the feature vectors for varying values of $k$ and use Dunn's index to choose the value of $k$ that leads to clustering with the highest Dunn's index.

\section{$k$-Means Clustering Algorithm}

1. Pick $k$ initial cluster centers by evenly dividing the range

2. For each feature vector: find the closest center $i$, label the vector as belonging to cluster $i$

3. For each of the $k$ clusters, recalculate the center from feature vectors having $k$ as label

4. If centers have changed goto step 2, else DONE.

\subsubsection{Dunn's Validity Index}

Dunn's validity index is a measure of cluster validity. Cluster validity measures are used to compare clusterings, indexing the clusters based on some criterion. Dunn's validity index is higher for a set of clusters with dense clusters that are well separated. Note that $k$-means clustering assumes one knows the number of clusters and provides initial guesses of their means. In order to try and find the correct number of clusters, $k$-means can be run several times with a range of values for $k$. We compare the clustering obtained for different values of $k$ by finding the Dunn's validity index for each clustering. We chose the clustering with the largest index. 
Dunn's validity index is defined as

$$
D_{k}=\min _{1 \leq i \leq k}\left(\min _{1 \leq j \leq k, j \neq i}\left(\frac{\delta\left(C_{i}, C_{j}\right)}{\max _{1 \leq m \leq k}\left\{\Delta\left(C_{m}\right)\right\}}\right)\right)
$$

where $C_{i}$ is cluster $i, \delta\left(C_{i}, C_{j}\right)$ is the intercluster distance (the distance between cluster $i$ and $j)$ and $\Delta\left(C_{m}\right)$ is the intracluster distance of $C_{m}$ (the distance within the cluster).

Clusters typically contain several feature vectors, therefore there are many options for representing the intercluster and intracluster distance. The intercluster distance can be represented using e.g. the minimum distance between feature vectors of each cluster, the maximum distance, the average or some other distance measure. Similarly for the intracluster distance, there are several possibilities.

To achieve a high index, the intercluster distance is maximized and the intracluster distance is minimized. With this in mind, we chose the minimum distance between feature vectors of each cluster as the intercluster distance and the maximum distance between feature vectors in the same cluster as the intracluster, thus providing a worst case distance for each. The intercluster distance is

$$
\delta\left(C_{i}, C_{j}\right)=\min _{x_{i} \in C_{i}, x_{j} \in C_{j}} d\left(x_{i}, x_{j}\right)
$$

where $d()$ is the Euclidean distance and $x_{m}$ is a feature vector in $C_{m}$. The intracluster distance is

$$
\Delta\left(C_{i}\right)=\max _{x_{i}, x_{j} \in C_{i}} d\left(x_{i}, x_{j}\right)
$$


By choosing the worst case distances for each, we aim to find the optimal clustering which will have compact clusters with maximum separation between clusters.

\subsubsection{Detection Results}

The detection results are evaluated using the detection rate and the false alarm rate. The detection rate measures the accuracy of the system in detecting desired objects and the false alarm rate assesses the system based on objects observed that are not of interest (i.e. not moving vehicles). These two rates together quantify the performance of the system. We use estimates of the detection and false alarm rates determined from the bounding boxes around the objects of interest [20].

The detection rate is the ratio of correctly detected objects to the actual objects (ground truth). We use area to measure the objects. The area of correctly detected objects is the area of intersection of observed objects and actual objects. The area of actual objects are estimated by manually marking the bounding boxes surrounding objects of interest (moving vehicles). Figure 6-3 is an example of the areas. In Figure 6-3, the observed object, the actual object, and the correctly detected area are shown above the example. The detection rate estimate is

$$
P_{d}(k)=\frac{\sum_{n_{o}=1}^{N_{d}(k)} a\left(n_{o}\right)}{\sum_{n_{i}=1}^{N_{a c t}(k)} a\left(n_{i}\right)},
$$

where $a(n)$ is the size of object $n$ in pixels, $N_{d}(k)$ is the number of objects from the intersection between the actual and observed objects of the system and $N_{a c t}(k)$ is the 
actual number of objects to be detected at the frame $k$.
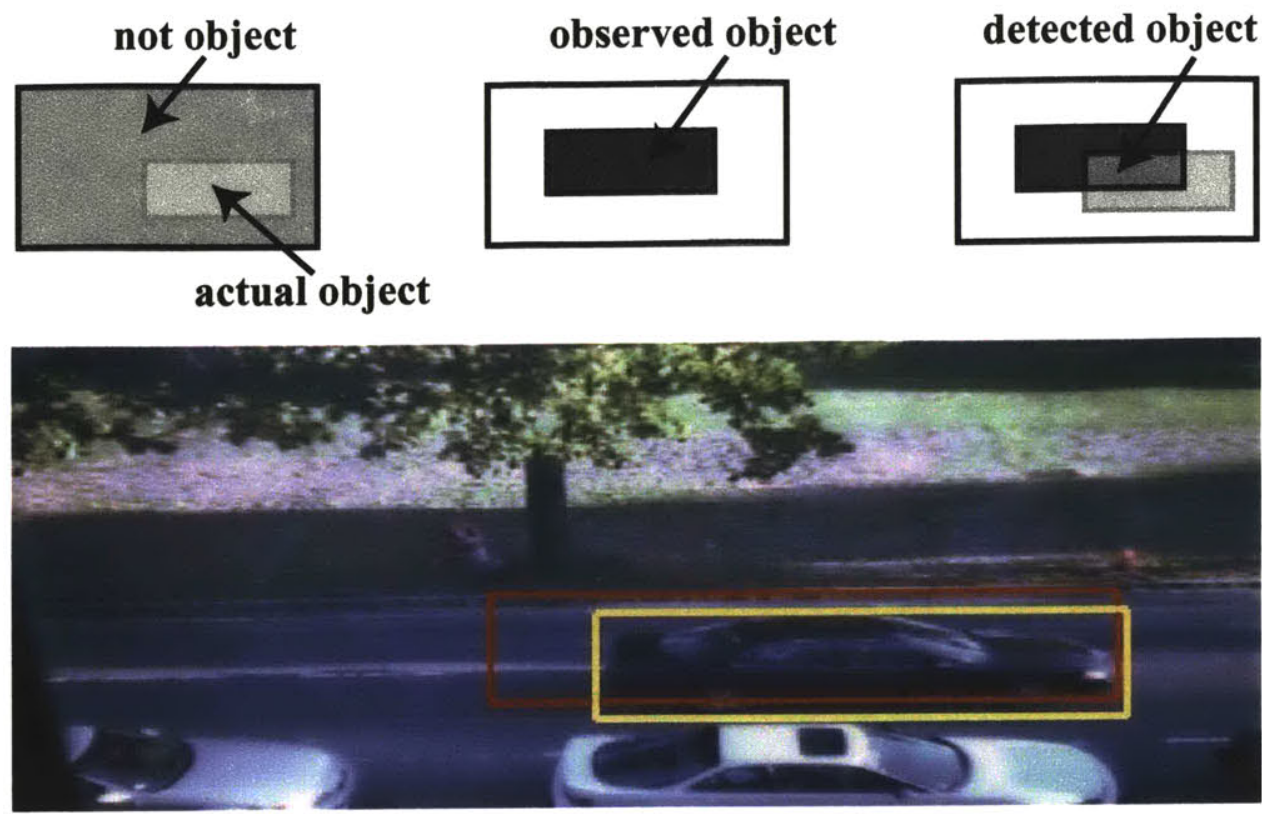

Figure 6-3: Example of actual, observed, and detected objects

The false alarm rate is an error measurement for the system defined as the ratio of the area of objects observed but that are not of interest, to the area of the image that does not contain objects of interest. The area of objects observed but that are not of interest is the observed objects (red box in Figure 6-3) minus the correctly detected objects (yellow box in Figure 6-3). The estimate of the false alarm rate is

$$
\begin{aligned}
& P_{f}(k)=\frac{\sum_{n_{i}=1}^{N_{o b s}(k)} a\left(n_{i}\right)-\sum_{n_{o}=1}^{N_{d}(k)} a\left(n_{o}\right)}{A_{f}(k)} \\
& A_{f}(k)=a(I(k))-\sum_{n_{i} \in N_{a c t}(k)} a\left(n_{i}\right)
\end{aligned}
$$

where $N_{o b s}(k)$ is the number of observed objects and $I(k)$ is the frame $k$. The false alarm rate coupled with the detection rate provide a good measure of the detection performance. 
The goal is to achieve a high detection rate with a low false alarm rate. We test the image sequences described in Section 4.3, a bounding box was manually selected around objects of interest (i.e. vehicles other than those that are parked). These bounding boxes represented the ground truth and were used to determine the detection and false alarm rate. Our system determines the bounding box surrounding the object of interest using $k$-means clustering along with Dunn's validity index for each frame.

There are 6308 images from the six days of images. Of these, $30 \%$ of the images have at least one object of interest. A receiver operating characteristic (ROC) curve provides a visual representation of the trade off between the detection rate and the false alarm rate. The average detection and false alarm rate for each frame is one point on the ROC curve. The thresholds for the size of the detected region and the number of contour pixels in an object were varied to produce the ROC curve. The ROC curve of the system is shown in Figure 6-4. A detection rate highlighted by a circle in Figure $6-4$ is $89.76 \%$ with false alarm rate, $1.95 \%$, where detection rate is high and false alarm rate is low. The misdetections in the system are primarily due to missing objects entirely. Raising the minimum size threshold increases the detection rate to $94.97 \%$ with a false alarm rate of $28.7 \%$. Accurate detection of the objects of interest (bounding boxes) also affects performance. Shadows impact the accuracy of the objects boundary detection. The detection rate can be raised by modeling the ground plane using depth information to remove shadows. False alarms occurred primarily due to the inaccuracy of bounding boxes, using color or shape information may help to reduce the false alarm rate. The performance of the current system 


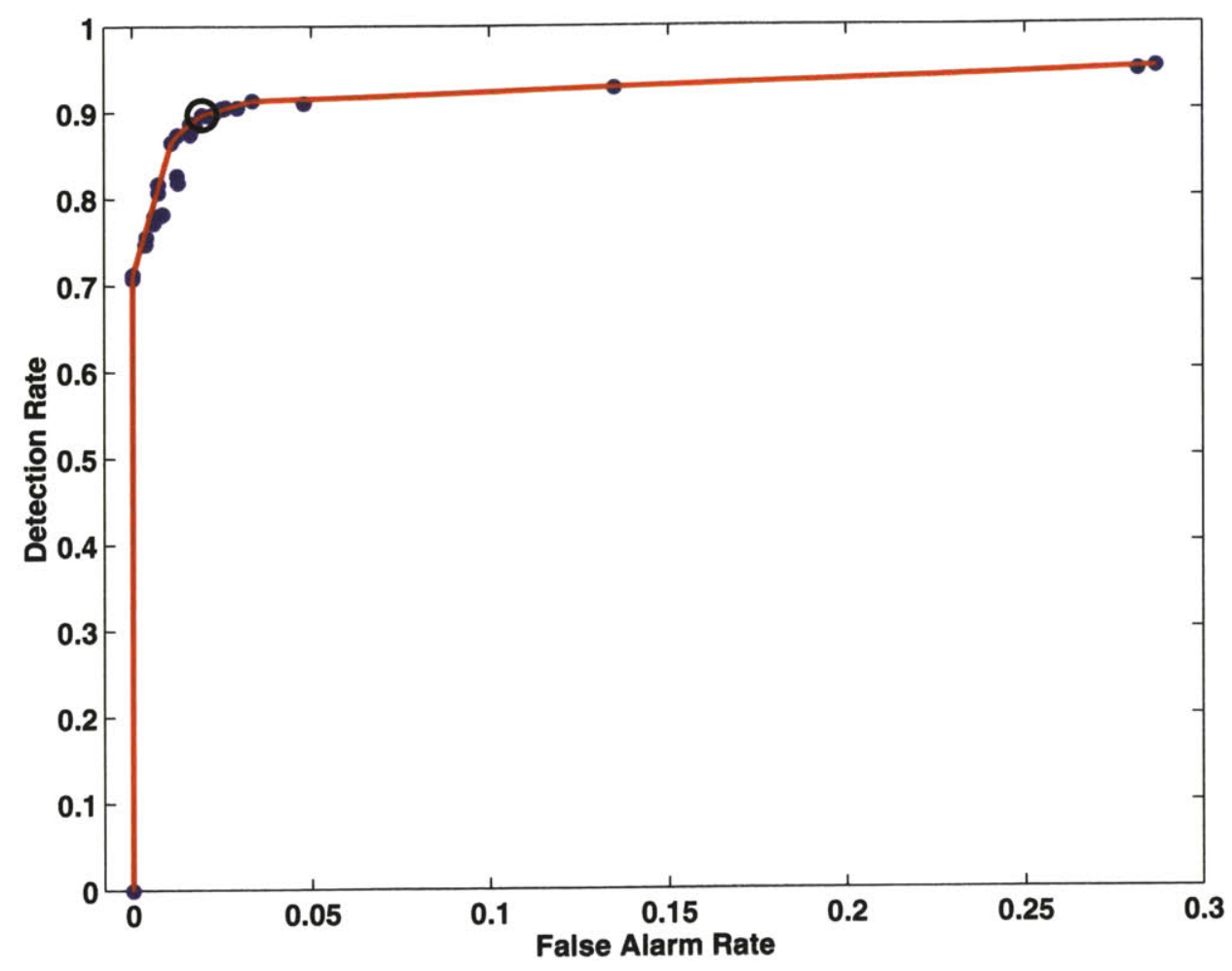

Figure 6-4: ROC Curve

demonstrates the feasibility of detecting objects in the compressed domain.

\subsection{Tracking}

Once a vehicle has been detected, vehicle counting and speed detection require tracking of the vehicle in subsequent images. Tracking involves identifying the motion of an object from one image to the next. Tracking provides a link between objects in two or more frames. Many tracking algorithms assume little or no changes in the shape of the object. Our system makes that same assumption. We assume the vehicle has only translational motion, and there is no significant warping or rotation of the vehicle image.

The tracking algorithm that has been implemented is shown in Figure 6-5. The 


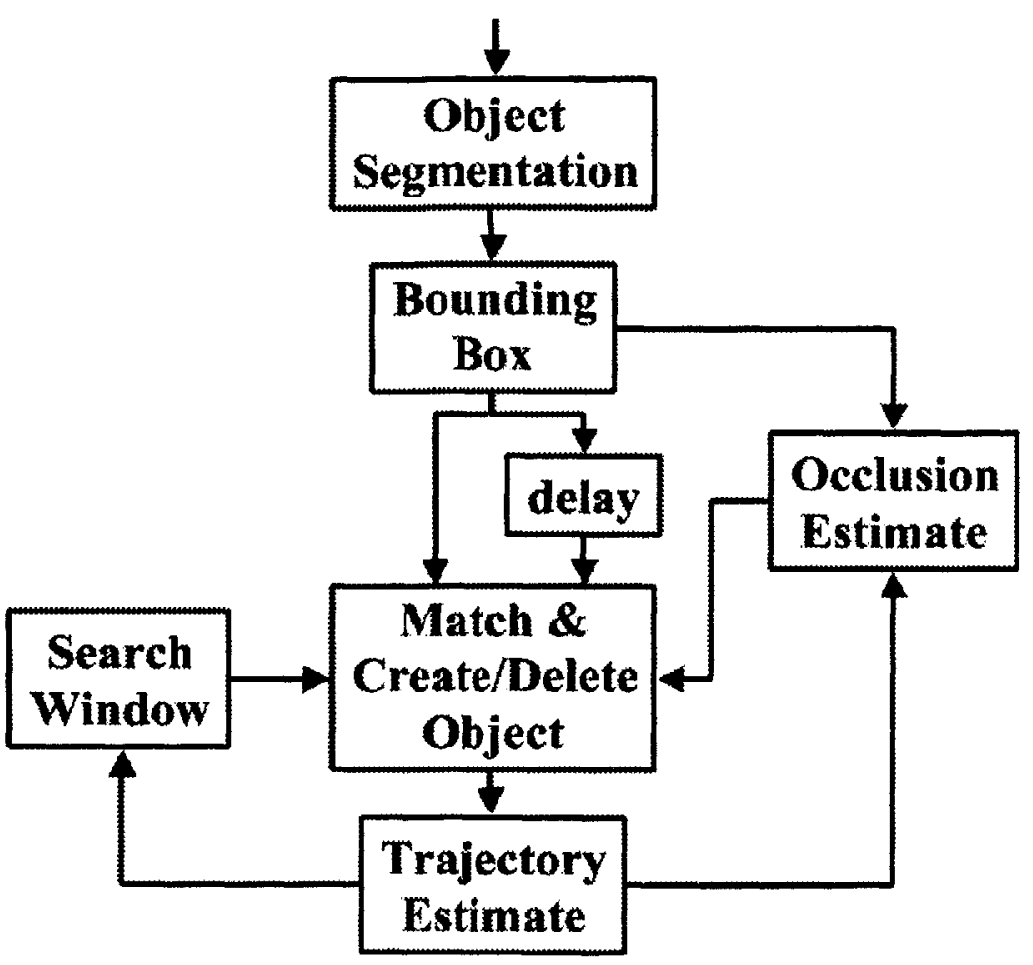

Figure 6-5: Tracking Algorithm

algorithm begins with the vehicle detection component (clustering contours). The vehicle is tracked by matching the vehicle from one image to the next. The motion vectors provide an initial guess of where the vehicle has moved to in the next image, partially occluded vehicles can not be tracked using only motion information. We use the position, area, depth and displacement information to determine the location of the vehicle in subsequent images.

\subsubsection{Matching Criteria}

The algorithm detects vehicles in each image and determines the trajectory of each vehicle by linking the objects from consecutive images. The matching criteria uses a matching score for each potential match. We begin with two sets of objects $P=$ $\left\{p_{1}, p_{2}, . . p_{n}\right\}$ and $C=\left\{c_{1}, c_{2}, \ldots c_{m}\right\}$, where $n$ is the number of objects detected in 
the first image and $m$ is the number of objects detected in the second image. The matching score is a weighted sum based on area, depth and motion. The matching score is defined as

$$
\operatorname{msc}(p, c)=\alpha\left(\frac{\mathrm{u}(\tilde{p}, c)}{\mathrm{a}(p)}\right)+\beta\left(1-\left|\frac{z_{p}-z_{c}}{z_{p}}\right|\right)+\gamma\left(1-\frac{\left|v_{p}-v_{c}\right|}{\left|v_{p}\right|}\right), p \in P, c \in C
$$

where $\alpha, \beta$, and $\gamma$ are weights summing to 1 . The motion vector in the first image gives an estimate of the objects location in the second image, we compare the estimated location from the first image with the detected location in the second image. This is the first term, where $\tilde{p}$ is object $p$ with an estimated location in the second image and $u(\tilde{p}, c)$ is the area of intersection of $\tilde{p}$ and $c$. The second term assumes the object does not move far from one image to the next, so we compare the depth, where $z_{p}$ is the depth of object $p$. Finally, we assume the motion of the object has not changed significantly from one frame to the next. The motion vector of object $p$ is $v_{p}$, and $\left|v_{p}\right|$ is the magnitude of the motion vector. Once a matching score is calculated for all $\mathrm{nm}$ possible matches, the algorithm continues as follows

\section{Matching Algorithm}

1. Find maxscore, $s=\max _{\forall p, c}\{\operatorname{msc}(p, c)\}$ and $\{\hat{p}, \hat{c}\}=\arg \max _{p, c} \operatorname{msc}(p, c)$

2. Loop until $s<t h_{m s c}$

- match $(\hat{p}, \hat{c})$ (objects $\hat{p}$ and $\hat{c}$ are considered the same object)

- Reset scores including $\hat{p}$ and $\hat{c}$ ( $\operatorname{set} \operatorname{msc}(\hat{p}, i)=0$ for $i=\{1, \ldots, q\}$ and $\operatorname{msc}(i, \hat{c})=0$ for $i=\{1, \ldots, n\})$

- Find $s=\max _{\forall p, c}\{\operatorname{msc}(p, c)\}$ and $\{\hat{p}, \hat{c}\}=\arg \max _{p, c} \operatorname{msc}(p, c)$

3. Done 
Using this algorithm, matches are generated from matching scores in descending order. If a match is not found for an object in the first subsequent image, then the object representation remains available for several more images, just in case occlusion is the reason a match has not been made.

A new object is created for tracking if an object in the second image has no match in the previous image. Objects are removed when the object is traveling out of view and the area of the bounding box is less than a preset threshold, or if after several images the object has not been redetected. This simple method of tracking may have problems with objects moving at the same speed in close proximity to each other. To solve this problem, models of vehicles and the road may be helpful in reducing this type error. 


\section{Chapter 7}

\section{Software Agents}

The system uses software agents to determine which images to transmit over the network. Software agents are autonomous programs. There are two variety of software agents, stationary and mobile. Mobile agents run on one or more remote computers, while stationary agents run on the originating computer. Agents are goal centered. Mobile agents are capable of gathering information from remote computers and other agents. Agents can communicate and collaborate with other agents to accomplish their goal. Research is currently being done to use mobile agents as personal assistants to schedule and reschedule meetings in a company environment. Mobile agents are also being used in e-commerce to find the best price and automatically purchase products.

Several different mobile agent environments have been created to facilitate the development of mobile agents, such as IBM Aglets, ObjectSpace Voyager, and General Magic Odyssey. Our system is implemented using IBM Aglets. IBM Aglets is a Java Package created to support mobile agents. An Aglet in the package is a base class for 
software agents. Mobile agents are confined to a limited area on remote computers. This area (execution environment) insures that the remote computer will not be attacked by rogue agents. An execution environment for the mobile agents offers a secure location for agents to be run and interface with the remote computer and any other agents in this environment. A view of Tahiti's (aglets' viewer) user interface is shown in Figure 7-1. The functionality of Tahiti includes

- Create - start agent

- Dispose - terminate agent

- Clone - start a copy of an agent

- Dispatch - send agent to remote computer

- Retract - return agent from remote computer

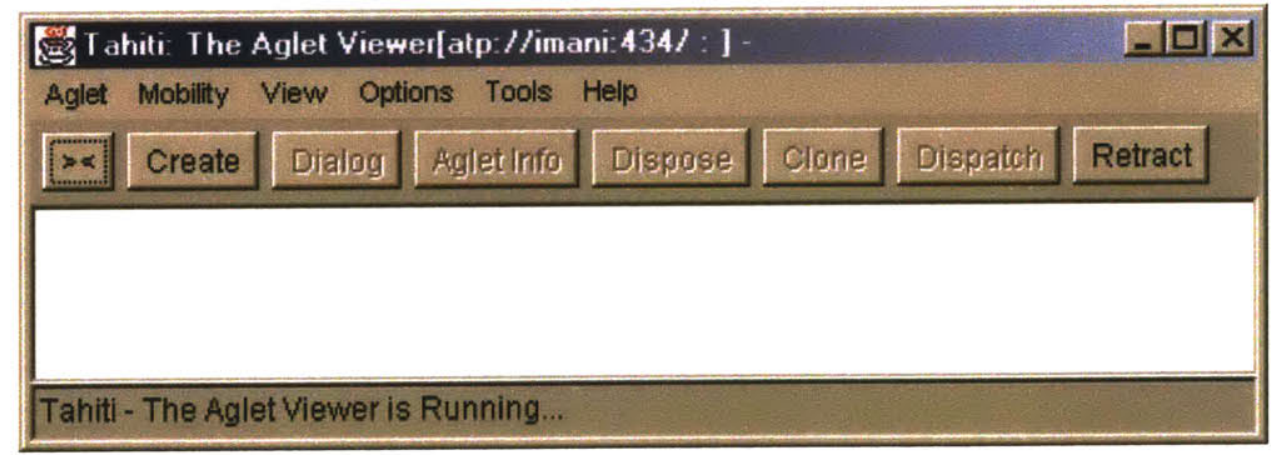

Figure 7-1: Aglet Environment

We use mobile agents to monitor camera outputs. The cameras classify each image based on the contents of the image. Before dispatching the agents to the camera, the agents are given a criteria, duration of monitoring and interval of checking. An agent checks the image content status at some set interval to determine if the criteria is met. If the criteria is met, the agent sends traffic information or the image from 
the camera location to the user. Images can be displayed in cycles, if the number of cameras to view is greater than the number of monitors. The user has the option of sending an agent to every location or any subset. The mobile agents act as filters for the network determining what information to send and when to send it.

\subsection{Implementation}

To implement the system, we focus on two locations, a camera with processor and a control center. The camera location accepts mobile agents, captures, processes, and saves images, as well as updates a file with current traffic information based on results from the processing of compressed image data. The camera allows mobile agents access to the traffic information and images. At the control center, mobile agents are dispatched to camera locations with preset goals and instructions. The control center displays traffic information and images sent by the mobile agent. We use Java classes to implement the agents and interface with operators at the control center.

\subsubsection{Classes}

The agents and the interface are extension of the Aglet and Frame class, respectively. The Aglet class has several methods that provide a foundation for both stationary and mobile agents. The interface contains several panels that each hold components to choose agent parameters, display images and dispatch agents. The details of both the Aglet class and the classes for the interface are provided below. 
A description of key components from several of the classes used in the mobile agent implementation are presented in this section. Below is a listing of classes used in the implementation:

Aglet abstract class from IBM Aglet package; extends Object implements Serializeable; base class for mobile agents

TrafficWatcher extends Aglet class; a stationary agent at the control center; dispatches TrafficNotifer with parameters and criteria

Notifier abstract class from IBM Aglet package; extends Aglet class; automatically dispatched upon creation; makes checks at a specified interval for a specified time duration

TrafficNotifler extends Notifier class; mobile agent sent to image sensor; checks if a file has been updated at a specified interval for a specified time duration; sends images if criteria is met 


\section{Aglet}

The Aglet class is available in the IBM Aglets package. The Aglet class is an abstract class used to implement mobile agents. The Aglet class extends the Object class and implements Serializable.

\begin{tabular}{|c|c|}
\hline Key Methods & Description \\
\hline clone() & clone aglet and proxy \\
\hline deactivate(duration) & $\begin{array}{c}\text { deactivate aglet; temporarily stop and remove, } \\
\text { resume after duration }\end{array}$ \\
\hline dispatch(URL) & dispatch aglet to URL \\
\hline dispose() & destroy and remove aglet \\
\hline getImage(URL, name) & get image (name) from URL \\
\hline handleMessage(Message) & handles the message from outside \\
\hline notifyMessage() & notifies a single waiting thread \\
\hline onCreation(Object) & initializes the new aglet \\
\hline onDisposing() & called when aglet is disposed \\
\hline run() & $\begin{array}{c}\text { aglet's thread; invoked upon creation, dispatch, } \\
\text { retraction, or activation of the aglet }\end{array}$ \\
\hline snapshot() & $\begin{array}{c}\text { snapshot will be activated if aglet is killed; } \\
\text { snapshot removed if dispose, dispatch, or } \\
\text { deactivate are invoked }\end{array}$ \\
\hline waitMessage() & waits until notified \\
\hline
\end{tabular}




\section{Notifier}

The Notifier class is an abstract class that extends the Aglet class. The Notifier is automatically dispatched. It checks (at the destination) at a specified interval for a specified time duration.

\begin{tabular}{|c|c|}
\hline Key State Variables & Description \\
\hline EXCEPTION & an exception has occurred \\
\hline EXPIRY & expired (based on duration) \\
\hline NOTIFICATION & notified master \\
\hline \hline Key Methods & Description \\
\hline & creates a notifier at the $U R L ;$ \\
with the name ( source); \\
context the notifier will be created; \\
a master aglet; $U R L$ of destination; \\
interval - time between checks; \\
duration is the life time of notifier; \\
master, destination, \\
interval, duration, stay, argument) \\
& at destination after notification; \\
& and argument is an argument object. \\
\hline handleMessage(Message) & handles message from outside \\
\hline onCreation(Object) & initializes the notifier \\
\hline
\end{tabular}

\section{Agents}

There are two types of agents used in the system, a TrafficWatcher and a TrafficNotifier. The TrafficWatcher is a master agent and is stationary at the control center. The TrafficWatcher dispatches one or more slave agents, TrafficNotifiers, to the cameras. The TrafficWatcher extends an Aglet class and the TrafficNotifier extends a Notifier class. A Notifier class is an extension of the Aglet class that continually checks if a file has been updated at a specified interval.

Java uses threads to enable concurrency. The TrafficWatcher has two threads, WatcherThread and ViewerThread, to enable the collection and display of images. 
When the TrafficWatcher is created, an interactive window is displayed shown in Figure 7-2. This window allows the TrafficWatcher to set the criteria, determine the

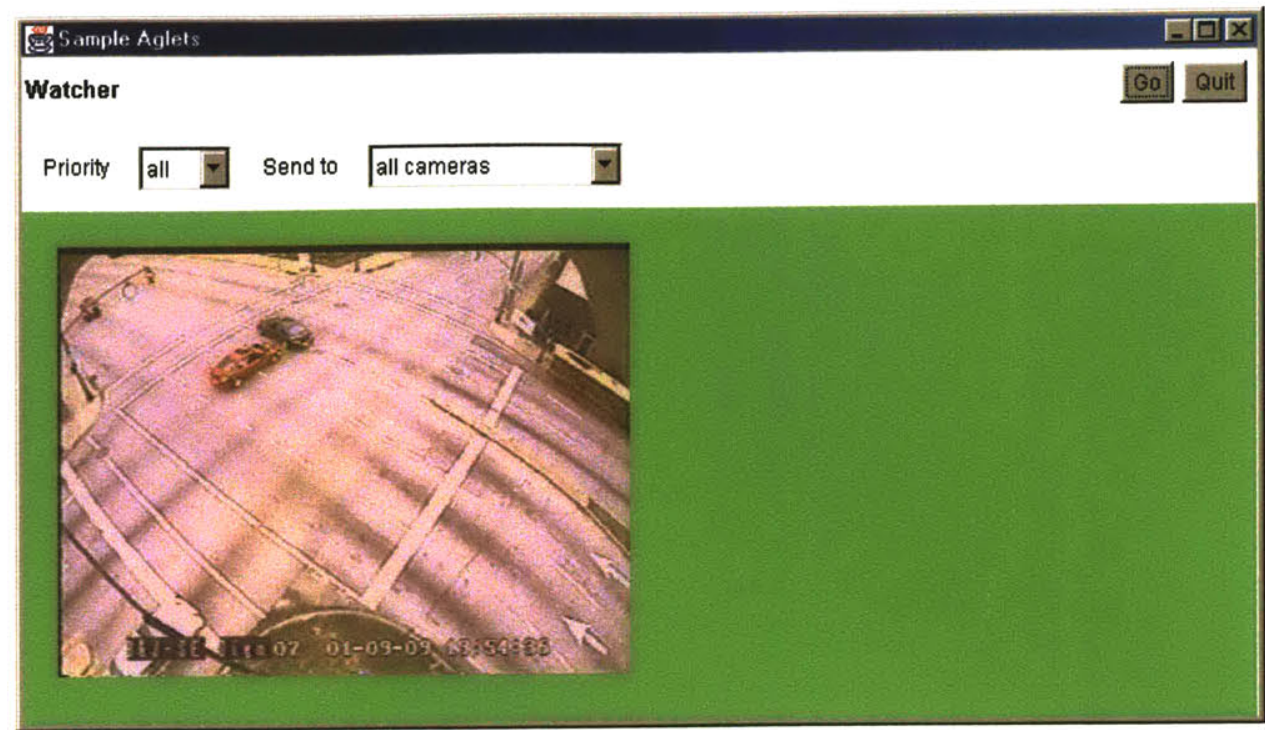

Figure 7-2: Interactive Window at the control center (Photo from KYTC / TRW / MELCO / TRIMARC)

image sensors of interest, and dispatch TrafficNotifiers. The lifespan of the TrafficNotifier and interval to check for traffic information updates are preset in this implementation. After the interactive window is displayed, the ViewerThread is started. This thread displays an image or refreshes the window and waits for a new image to be sent to the control center.

The TrafficNotifier, once dispatched to the appropriate image sensor, checks the traffic information at the image sensor. The TrafficNotifier continues to check the traffic information on a preset interval until it is disposed either by the operator at the control center or if it has reached the duration time. The TrafficNotifier sends an image or information to the control center, if the criteria has been met. Figure 7-3 shows a simple interaction between the control center and the image sensor. The 


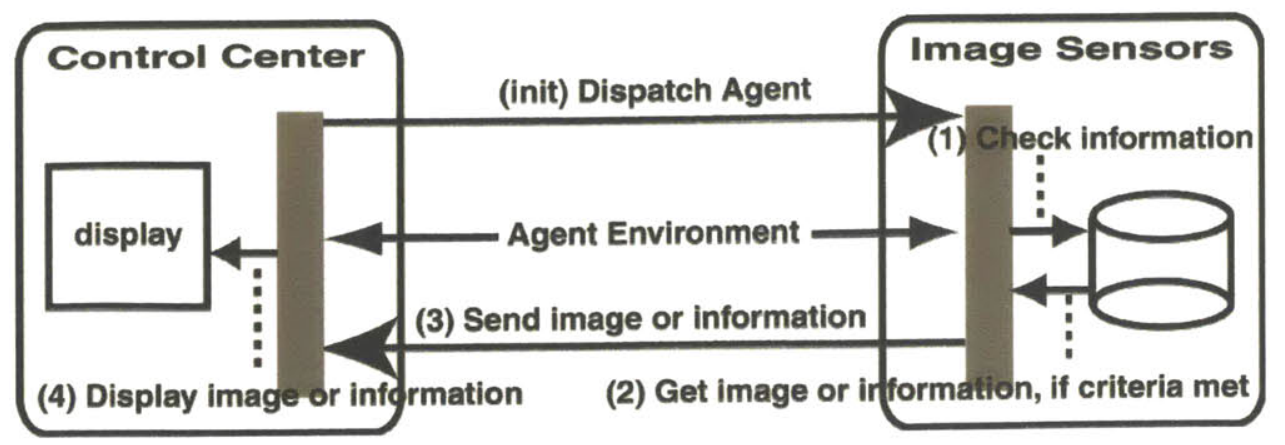

Figure 7-3: Interaction between control center and image sensor

TrafficNotifier runs in the agent environment at the image sensor and has access to traffic information and images. Images are sent to the TrafficWatcher at the control center. The control center displays the images and information on the available monitors.

\section{Control Center Interface}

The interface for the control center is an interactive window where operators specify the parameters and criteria for mobile agents and receive traffic information and/or images based on operator input. The interactive window is a TrafWindow class that extends a Frame class. There are panels to set TrafficNotifier parameters, a panel to display traffic images, and panels for buttons, messages, and errors in the TrafWindow. The window shown in Figure 7-2 has a "Go" and "Quit" button. The "Go" button dispatches one or more TrafficNotifier agents and "Quit" disposes the agents and closes the window. The parameters and criteria for TrafficNotifier are

- check interval* - time interval to check the traffic information

- duration* - lifespan of the TrafficNotifier

- stay* - does the TrafficNotifier remain after notification 
- priority - criteria to determine when to send images

- camera location - location(s) where the TrafficNotifier(s) will be sent

where $*$ are parameters preset, not shown in the window. These parameters determine the functionality of the TrafficNotifier agent(s). Panels that display text information are used for messages and errors. There is also a panel to display traffic images. To simulate several monitors, we display all of the monitors in an image panel. The number of monitors (images to display) in the window is preset, corresponding to the features of the control center.

\subsection{Enhancements}

We chose to use mobile agents to select images that are to be transmitted over the network. Mobile agents can be used in many different applications. We implemented a simple application to demonstrate the feasibility of mobile agents as a filter for the network. Modifications to the system or other implementations could be applied to improve performance and increase functionality of the system.

\subsubsection{Image Management}

Currently, we show all images sent to the control center and cycle through the images. Although the filtering described above does decrease the transmission load, since we have only sent images which meet a desired criteria, we can reduce the transmission load even further. We can limit the number of images sent over the network by providing mobile agents with the current number of available monitors. Mobile agents 
can work together to determine which images are sent to the control center.

\subsubsection{Route Guidance}

A more complex system involving the collaboration of mobile agents can be developed to determine the best route between two locations and an estimated travel time. Several agents can be dispatched along several routes and travel some length of the route to find estimates of travel times. Agents can communicate with each other to distribute processing and determine the best route. The best route can be given to the driver before starting to travel. Other applications, such as on-route guidance based on current traffic information and traffic flow pattern analysis can also be accomplished using mobile agents. Applications useful to drivers and traffic control centers can be developed to improve transportation systems. 


\section{Chapter 8}

\section{Additional Applications}

So far, our focus has been on vehicle detection and tracking from compressed image data. In this chapter, we demonstrate three additional applications of processing compressed image data, vehicle detection by color and lane detection. The algorithms to implement these applications are simple and effective, due to features of the compressed image data. We designed algorithms that demonstrate the feasibility of processing compressed image data for such applications.

\subsection{Vehicle Detection by Color}

Feature selection for object detection is dependent on the view and the objects of interest. In Chapter 6, we described how to detect vehicles using motion vectors. In this section, we demonstrate the detection of a vehicle in an image based on color.

Vehicle detection by color can be accomplished through color histogram matching. In our case, the size of the vehicle in the image is relatively small, therefore we chose 
to use the dominant color of the vehicle rather than color histogram matching. We also chose a view where the vehicles are not occluded. To detect the vehicle based on color, we group all contours with the search color on either side of the contour. This produces a large bounding box that includes the vehicle of interest, but is not tightly bound. We reduce the bounding box by eliminating sparse regions around the borders. A region is determined to be sparse based on the percentage of pixels considered part of the vehicle. Figure 8-1 shows the yellow vehicle detected by color.

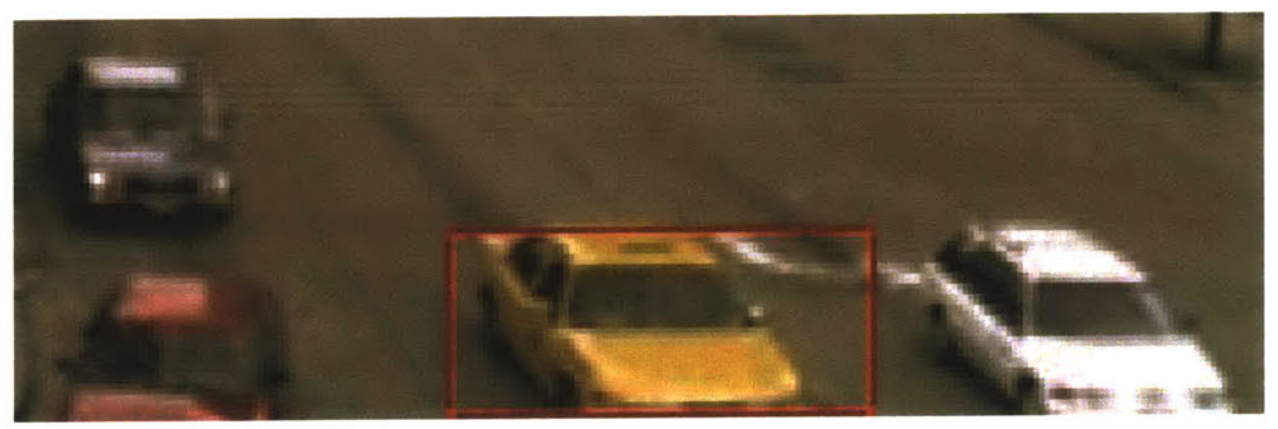

Figure 8-1: Detect yellow vehicle based on color

\subsection{Lane Detection}

Lane detection is the process of detecting the lane boundaries from one or more images. Lane detection is used to improve the reliability of automatic cruise control, collision warning systems, and automatic driving systems. Lane detection provides these systems with additional information to aid in the decision process. Lane detection helps to reduce the number of false alarms in a collision warning system by focusing on vehicles that are in the same lane, rather than on vehicles that are a certain distance away. Automatic cruise control and driving systems are able to safely 
maintain the desired speed by monitoring vehicles in the same lane. Lane detection clarifies the position of nearby vehicles and provides a better awareness of the surroundings.

To test our lane detection algorithm, we focused on detecting lane markings on a highway. We mounted two cameras horizontally on the dashboard. The view of the highway is limited to the road and vehicles. A few trees are visible along the borders of the highway.

We detect the lanes by detecting the vehicles on the road and eliminating them from the scene as shown in Figure 8-2. To detect the vehicles in the image we use

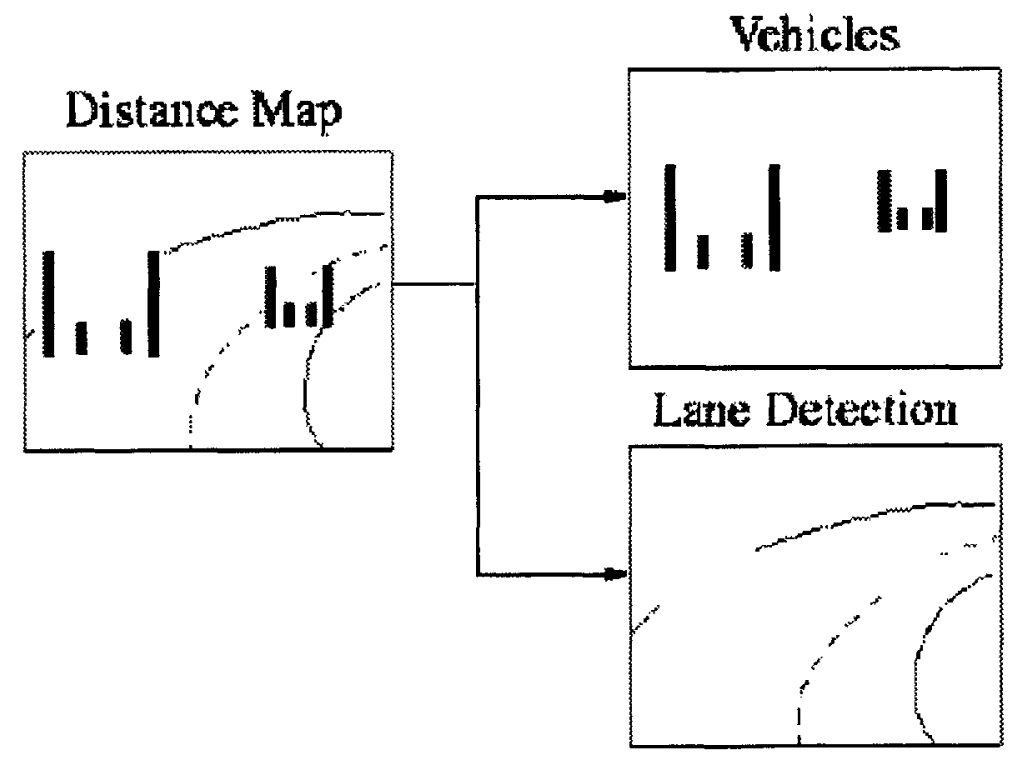

Figure 8-2: Lane Detection

the histogram of edge depth information. The vehicles are assumed to be regions where there is a strong peak in the histogram (large number of pixels at the same distance). We eliminate the vehicle by removing the depths that correspond to the vehicle, leaving the lane markings. Although, when we remove the vehicle we are potentially removing a portion of the road, it is typically a relatively small part. 
In this case, what would normally be considered noise in the histogram for purposes of object detection contains the lane markings. Lane markings which are close in distance to the camera will have more pixels than lane markings further away, therefore the threshold is a sloping line in the histogram. A horizontal line is not a sufficient threshold to separate the vehicles from the lane markings. Figure 8-3 shows a histogram with this thresholding scheme applied to detect lane markings.

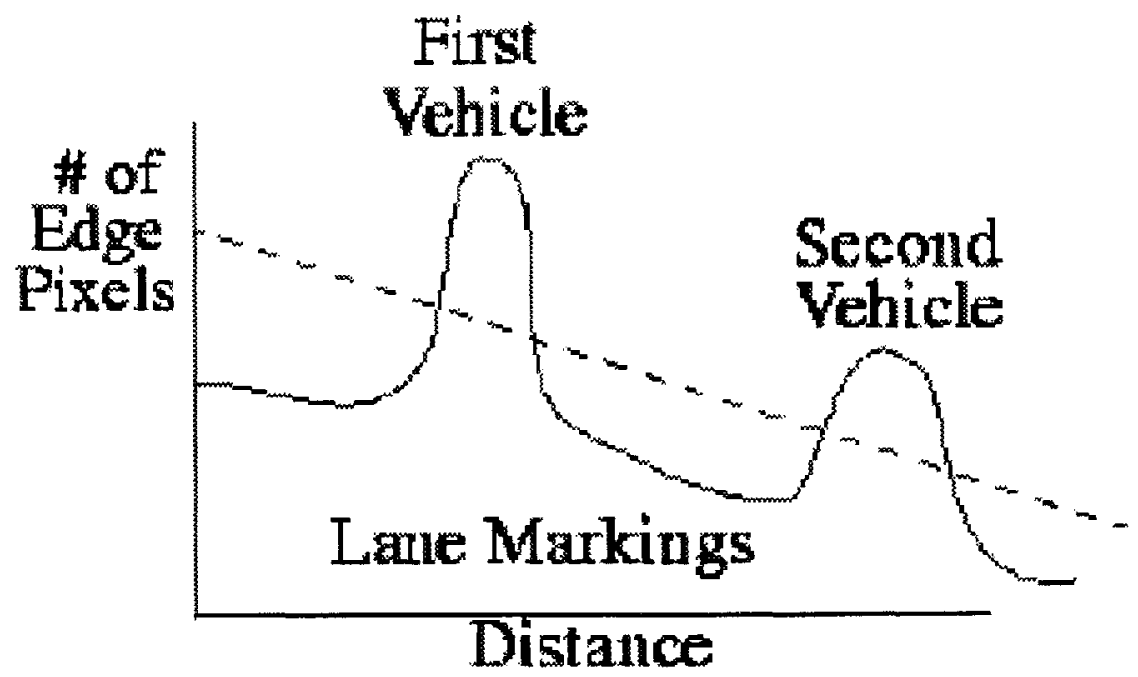

Figure 8-3: Histogram with thresholding

The objects are detected and eliminated from the scene, leaving the lane markings along with some noise. Figure 8-5 is the histogram of the depth map in Figure 8-4. Figure 8-6 is the depth map with the vehicle removed leaving the lane markings.

Once the vehicles are removed, the lane markings are determined by approximating the curve using a sequence of linear approximation from the pixels remaining in a limited search area. Searching areas are limited to the lower left and right of images. A linear approximation is made restricting the direction and slope of the line. Figure 8-7 shows the right lane marker being detected using this method. The left lane 


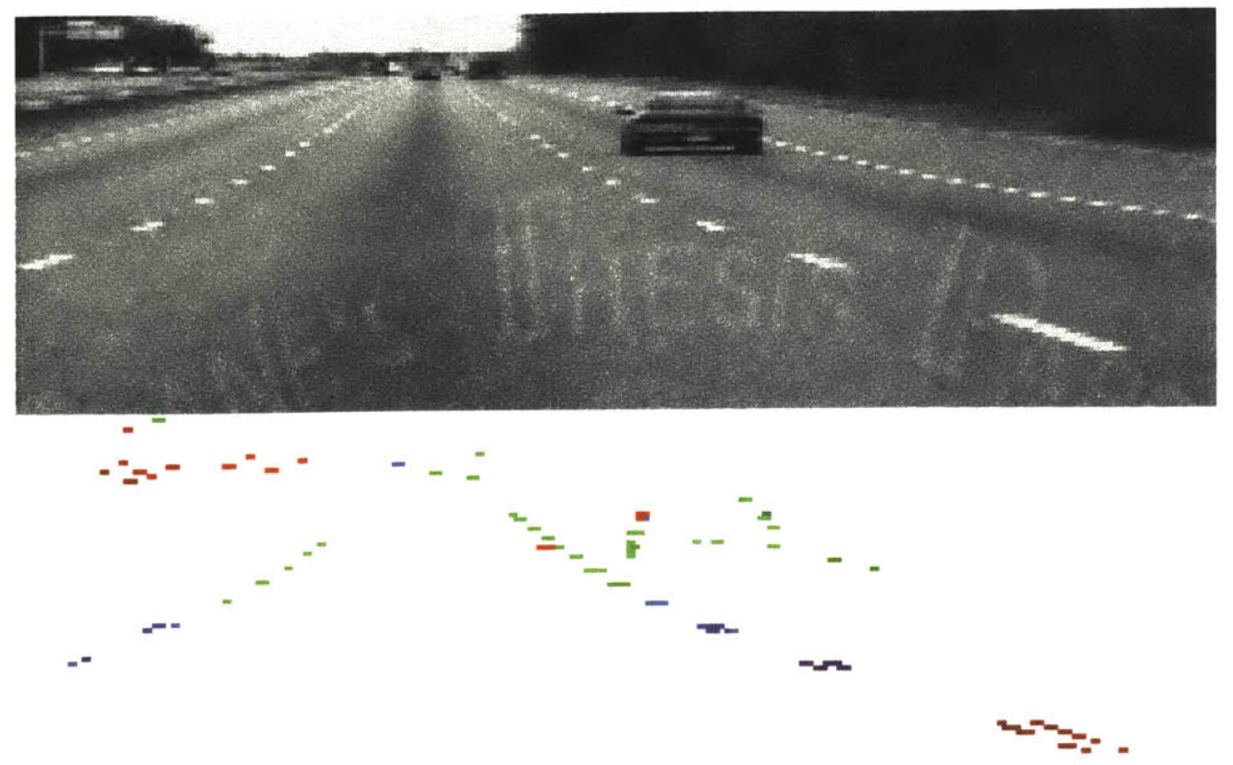

Figure 8-4: Original image with depth map

marker was not detected due to the search area used. The left lane marker began more than a third of the way above the bottom of the image. The left marker may be detected by improving the initial search area based on the location of the cameras or using an estimate of the width of the lane to alter the search area. The lane detection of the right marker demonstrates the feasibility of processing contour-based compressed image data and detecting lane markers using edge depth information. 


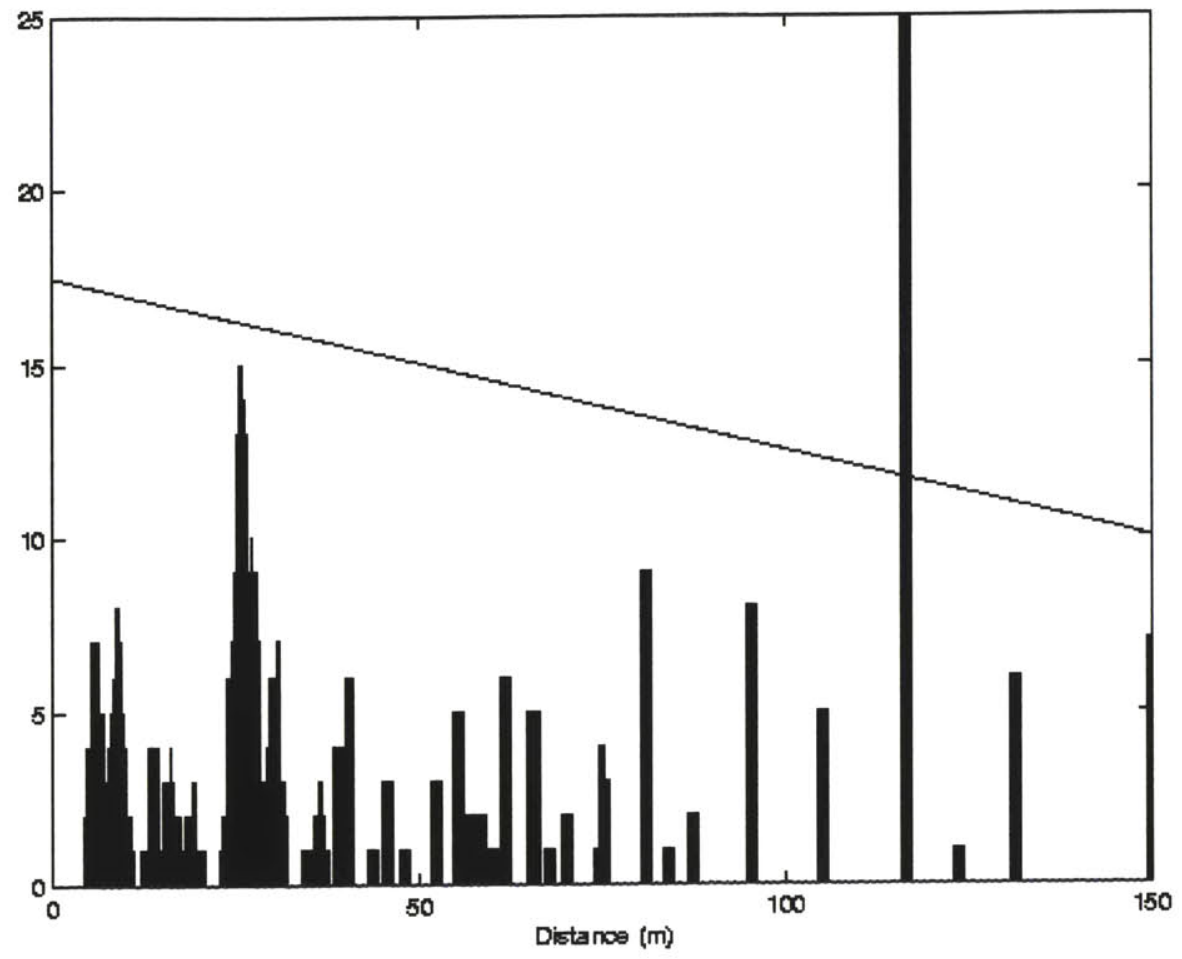

Figure 8-5: Depth histogram with thresholding

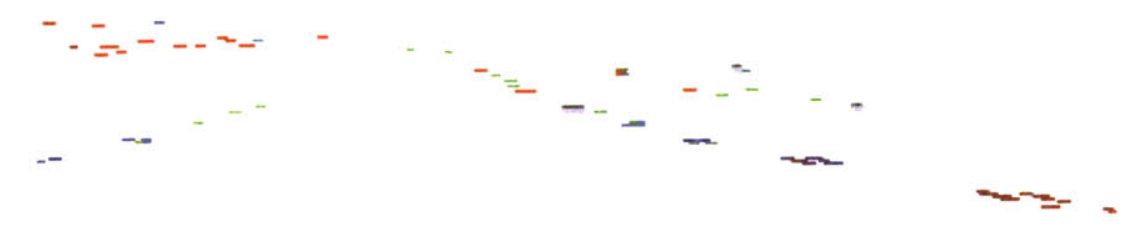

Figure 8-6: Vehicles eliminated from scene

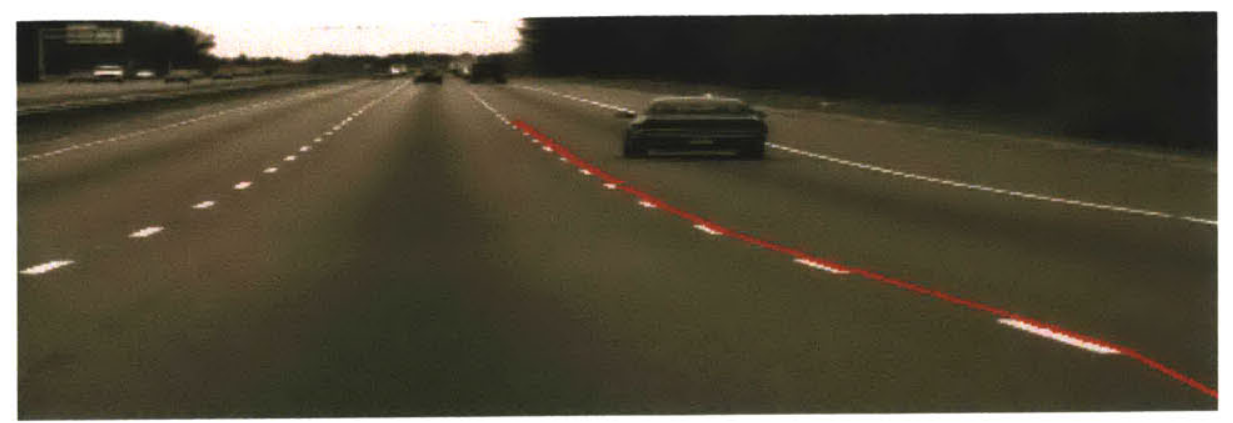

Figure 8-7: Lane Detected 


\section{Chapter 9}

\section{Conclusion}

In this thesis, the goal was to find ways to reduce transmission loads of networks dedicated to the continual transmission of images. We focused on Intelligent Transportation Systerns, specifically traffic monitoring, where hundreds of images are continually sent from image sensors to traffic control centers. Traffic control centers typically have 10-20 monitors dedicated to cycling through current traffic images. The continual transmission of hundreds of images is an unnecessary load. We presented two methods of reducing transmission over the network, 1) compression of images and 2) filtering the network using mobile agents. The mobile agents depend on knowledge of the contents of the images to efficiently filter the network. Therefore, the images must be processed at the image sensors before being sent over the network.

Our compression method retains edge, color, depth, and motion information to facilitate further processing of the images. The compression method is a modification of Mizuki's compression method. There are three modifications, 1) addition of depth information (2D to 3D), 2) a different contour coding (DCC to MDCC), and 3) a 
change in motion vectors (binary block matching of entire image to block matching based on contours). The depth information enhances the representation of the image, providing an additional feature for processing. The modification to the contour coding allows for the incorporation of splits into the contour representation and increases the compression of the images. The switch from block matching to contour based motion vectors increases the accuracy of the motion estimates and improves object detection. The compression method is designed to compress the image as much as possible, while enable processing of the images to determine the contents.

A mobile agent decides if or when to send an image, based on the contents. The operator at the control center, via mobile agents, specifies the conditions to send images over the network. Images are only sent when the criteria is met, thus reducing the number of images sent over the network. Images are processed to determine if the conditions are met.

Algorithms were developed to determine the contents of the images. The algorithms utilize the contour information from the compressed image data to perform object detection and tracking. We demonstrated the ability to process compressed image data by detecting and tracking vehicles in complex images. We focused on moving vehicles that were partially occluded by parked vehicles.

Vehicle detection by color and lane detection, via processing of compressed images, are also discussed with preliminary results. We detected lane markers using depth information from the compressed image data. Collision warning systems, cruise control and other driving assistive systems can use the location of lane markers to enhance their performance. We also presented a simple image database retrieval system that 
can be useful in searching for images based on content.

Our focus has been directed to ITS, but the entire system and/or components can be tailored to other systems. The compression method can be used to compress images where processing of the image is more important than viewing the image. The compression method is most useful in systems where determining the contents of the image through processing is the goal. The images can be compressed and stored for future processing. Mobile agents can be used to filter any network, specifically an image sensor network where there is a large amount of data being continually transmitted. The mobile agents along with the compression method can be used to reduce transmission loads based on image content and user preference.

\subsection{Future Research}

The research can be expanded by improving the quality of the decompressed image. Adjustments, such as methods to reduce the blur caused by smoothing and a higher quality color quantization, will increase the quality of the image. A method to reduce the search area for motion vector calculations will increase speed, using, for example, a predictive coding method that takes the nearby motion vectors into account.

Additional functionality in the mobile agents, such as collaboration, will enable our system to be used in more applications. Image management, as discussed in section 7.2.1, will further reduce the number of images sent over the network. The user interface can be enhanced to provide operators with more control over the mobile agents. 
The system needs to be tested in both simulated and real world situations. Additional processing, such as accident/incident detection, needs to be implemented. Route guidance and surveillance systems can also be implemented and tested.

Our system is designed to be used in other surveillance systems. Corporate buildings and airports are examples where our system could be implemented and used to efficiently monitor surroundings. The compression method and mobile agents work together to decrease transmission load and provide operators only images of importance. In large systems, with a significant number of image sensors and a small number of monitors, it is important to insure that images are viewed based on content rather than randomly chosen. 


\section{Bibliography}

[1] B. Bascle, R. Deriche. Region tracking through image sequences, IEEE 5th International Conference on Computer Vision, pp. 302-305, 1995.

[2] J. Bergendahl, A Computationally Efficient Stereo Vision Algorithm for Adaptive Cruise Control, Master's Thesis, May 1997.

[3] J. Bergendahl, I. Masaki, B.K.P. Horn, Three-camera stereo vision for intelligent transportation systems, Proceedings of SPIE's Photonics East '96 Symposium, Boston, MA, November 18-22, 1996.

[4] M. Bertozzi, A. Broggi, A. Fascoli, S. Nichele, Stereo Vision-based Vehicle Detection, Proceedings of the IEEE Intelligent Vehicle 2000 Symposium, p. 39, October 2000.

[5] D. Beymer, J. Malik. Tracking Vehicles in Congested Traffic Proceedings of the 1996 IEEE Intelligent Vehicles Symposium, September 1996.

[6] D. Beymer, P. McLauchlan, B. Coifman, and J. Malik, A Real-time Computer Vision System for Measuring Traffic Parameters, IEEE Conference Computer Vision and Pattern Recognition, pp. 495-501, 1997. 
[7] V. Bhaskaran, K. Konstantinidies, Image and Video Compression Standards Algorithms and Architectures, Kluwer Academic Publishers 1995.

[8] Bureau of Transportation Statistics, National Transportation Statistics 2002 BTS02-08, US Department of Transportation, 2002.

[9] California Alliance for Advanced Transportation Systems (CAATS), Intelligent Transportation Systems (ITS) Deployment Initiatives Project, February 2000.

[10] Y. Chen, Highway Overhead Structure Detection Using Video Image Sequences, IEEE Transactions on Intelligent Transportation Systems, Vol. 4, Issue 2, pp. 67-77, June 2003.

[11] K-L. Chung and K-B. Hong, Level Compression-Based Image Representation and Its Applications, Pattern Recognition, Vol. 31, No. 3, pp. 327-332, 1998.

[12] W. Cox, New Urban Rail in America: Miniscule Impact on Congestion, The Public Purpose, No. 23 (September 1998), http://www.publicpurpose.com/pp-rtranf.htm.

[13] U.Y. Desai, M.M. Mizuki, I. Masaki, and B.K.P. Horn, Edge and mean based image compression, MIT Artificial Intelligence Memo: AIM-1584.

[14] M. Devy, A. Giralt, and A. Martin-Hernandez, Detection and Classification of Passenger Seat Occupancy using Stereovision, Proceedings of the IEEE Intelligent Vehicle 2000 Symposium, p.714, October 2000. 
[15] A.W. Drake, Fundamentals of Applied Probability Theory, McGraw-Hill, New York, 1967.

[16] R.O. Duda, P.E. Hart, D.G. Stork, Pattern Classification and Scene Analysis, Part 1: Pattern Classification, Wiley, 2nd edition, 2001.

[17] M. Eden and M. Kocher, On The Performance of a Contour Coding Algorithm in the Context of Image Coding Part I: Contour Segment Coding, Signal Processing, Vol 8, pp. 381-386, 1985.

[18] FHWA, Comprehensive Truck Size and Weight Study: Summary Report for Phase I-Synthesis of Truck Size and Weight (TS\&W) Studies and Issues, United States Department of Transportation, March 1995.

[19] N.J. Ferrier, S.M. Rowe, A. Blake. Real-Time Traffic Monitoring Proceedings of the Second IEEE Workshop on Applications of Computer Vision, pp. 81-88, December 1994.

[20] G.L. Foresti and C.S. Regazzoni. New Trends in Video Communications, Processing and Understanding in Surveillance Applications IEEE International Conference on Image Processing 2001, October 2001.

[21] U. Franke and I. Kutzbach, Fast Stereo based Object Detection for Stop and Go Traffic, IEEE Intelligent Vehicle Symposium 1996, p.339, 1996.

[22] U. Franke and A. Joos, Real-time Stereo Vision for Urban Traffic Scene Understanding, Proceedings of the IEEE Intelligent Vehicle 2000 Symposium, p.273, October 2000. 
[23] K. Fukui, H. Nakai, Y. Kuno. Multiple Object Tracking System with Three Level Continuous Processes, IEEE Workshop on Applications of Computer Vision, pp. 19-27, November 1992.

[24] R. Gangisetty, C.G. Ardillo. Advanced Traffic Management System on Interstate 476, Proceedings of the 1995 Annual Meeting of ITS America, pp. 579-588, March 1995.

[25] D.M. Gavrila and V. Philomin. Real-time Object Detection for "Smart" Vehicles, Proceedings of IEEE International Conference on Computer Vision, pp. 87-93, Kerkya, 1999.

[26] A. Goldsmith and G.W. Cleven. Highway Electronic Systems - Today and Tomorrow, IEEE Transactions on Vehicular Technology, Vol. VT-19, pp. 161-167, January 1970.

[27] E. Gose, R. Johnsonbaugh and S. Jost. Pattern Recognition and Image Analysis, Prentice Hall, Inc., 1996.

[28] J. Guo and A. Zhang, E. Remias and G. Sheikholeslami, Image Decomposition and Representation in Large Image Database Systems, Journal of Visual Communication and Image Representation, Vol. 8, No. 2, pp. 167-181, June 1997.

[29] R. W. Hamming, Coding and Information Theory, Prentice-Hall, Inc., 1980.

[30] D. Hankerson, G. A. Harris, P. D. Johnson, Jr., Introduction to Information Theory and Data Compression, CRC Press 1998. 
[31] R. Hartley, In Defence of the 8-point Algorithm, Proceedings of the Fifth International Conference on Computer Vision, pp. 1064-1070, June 1995.

[32] H. Hattori, Stereo for 2D Visual Navigation, Proceedings of the IEEE Intelligent Vehicle 2000 Symposium, p.31, October 2000.

[33] W.J. Heng and K.N. Ngan. An Object-Based Shot Boundary Detection Using Edge Tracing and Tracking, Journal of Visual Communication and Image Representation, September 2001, vol. 12, no. 3, pp. 217-239(23).

[34] T. Hill, M. Kyng, S.S. Shyne, J.L. Lovelace, E.G. Roberts, M.D. Schauer. Traffic Flow Visualization and Control Improves Traffic Data Acquisition and Incident Detection, Proceedings of the 1996 Annual Meeting of ITS AMERICA, pp. 370377, April 1996

[35] B.K.P. Horn, Robot Vision, The MIT Press, 1986.

[36] B.K.P. Horn, Relative Orientation, International Journal of Computer Vision, Vol. 4, No. 1, pp. 59-78, January 1990.

[37] B.K.P. Horn, Relative Orientation Revisited, Journal of the Optical Society of America, Vol. 8, pp. 1630-1638, October 1991.

[38] Iowa Department of Transportation, Statewide Transit Intelligent Transportation Systems Deployment Plan: Working Paper 2 - What are Intelligent Transportation Systems (ITS) Technologies for Transit?, May 15, 2002. 
[39] Intelligent Transportation Society of America (ITS America), National Intelligent Transportation Systems Program Plan: A Ten-Year Vision, 2002.

[40] L. Kaminski, J. Allen, I. Masaki, and G. Lemus, A Sub-Pixel Stereo System for Cost-Effective Intelligent Vehicle Applications, IEEE Proceedings of the Intelligent Vehicles '95 Symposium, pp. 7-12, September 1995.

[41] T. Kanade, A Stereo Machine for Video-Rate Dense Depth Mapping and Its New Applications, Proc. ARPA Image Understanding Workshop, pp. 805-814, Palm Springs, 1996.

[42] A. Kaup and T. Aach, Coding of Segmented Images Using Shape-Independent Basis Functions, IEEE Transactions on Image Processing, Vol. 7, No. 7, July 1998.

[43] N. Kehtarnavaz, C. Huang, T. Urbanik, Video Image Sensing for a Smart Controller at Diamond Interchanges, Proceedings of the 1995 Annual Meeting of ITS America, pp. 447-451, March 1995.

[44] C. Knoeppel, A. Schanz, and B. Michaelis, Robust Vehicle Detection at Large Distance Using Low Resolution Cameras, Proceedings of the IEEE Intelligent Vehicle 2000 Symposium, p. 267, October 2000.

[45] D.J. Kriegman, E. Triendl, and T.O. Binford, Stereo Vision and Navigation in Buildings for Mobile Robots, IEEE Transactions on Robotics and Automation vol. 5, no. 6, p. 792, December 1989. 
[46] M. Lay, The past and future of Intelligent Transport Systerns, The Royal Society of Victoria, 1997.

[47] R.S. Ledley, The processing of medical images in compressed format, SPIE Vol. 1905, pp. 677-687, August 1993.

[48] J.A. Lindley, Quantification of Urban Freeway Congestion and Analysis of Remedial Measures, United States Department of Transportation Federal Highway Administration FHWA/RD-87/052, October 1986.

[49] R.P. Maccubbin, B.L. Staples, M.R. Mercer, Intelligent Transportation Systems Benefits and Costs 2003 Update, United States Department of Transportation Federal Highway Administration, May 2003.

[50] J. Malik, S. Russell, J. Weber, T. Huang, and D. Koller. A Machine Vision Based Surveillance System for California Roads, PATH project MOU-83 Final Report, 1995.

[51] S. Marshall. Application of Image Contours to Three Aspects of Image Processing: Compression, Shape Recognition and Stereopsis, IEE Proceedings-I Communications Speech \& Vision, Vol. 139, No. 1, pp. 1-8, Feb. 1992.

[52] B. Gianni and A. Moore, A Case for Intelligent Transportation System (ITS) Telecommunications Analysis (Maryland State Highway Administration's ITS Telecommunications Study), 1995.

[53] I. Masaki. Industrial Vision Systems Based on Application-Specific IC Chips, IEICE Transactions, Vol. E 74, No. 6, June 1991. 
[54] L. Matthies, A. Kelly, T. Litwin, and G. Tharp, Obstacle Detection for Unmanned Ground Vehicles: A Progress Report, Proceedings of the IEEE Intelligent Vehicles 1995 Symposium, p. 66, September 1995.

[55] T.L. Mengko, T. Adiono, H. Setyawan, and R. Setiadarma, Design and implementation of object detection and classification system based on deformable template algorithm, The 1998 IEEE Asia-Pacific Conference on Circuits and Systems, pp. 311-314, November 1998.

[56] M.M. Mizuki, Edge Based Video Image Compression for Low Bit Rate Applications, M.S.E.E Thesis, MIT, Cambridge, September 1996.

[57] A. N. Netravali and B. G. Haskell, Digital Pictures Representation, Compression, and Standards, Plenum Press 1995.

[58] Y. Ninomiya, S. Matsuda, M. Ohta, Y. Harata, and T. Suzuki, A Real-time Vision for Intelligent Vehicles, Proceedings of the Intelligent Vehicles '95 Symposium, 1995, pp. 315-320, September 25-26.

[59] M. Nishigaki, M. Saka, I. Aoki, H. Yuhara, and M. Kawai, Fail Output Algorithm of Vision Sensing, Proceedings of the IEEE Intelligent Vehicle 2000 Symposium, p.581, Octember 2000.

[60] K. Onoguchi, N. Takeda, and M. Watanabe, Planar Projection Stereopsis Method for Road Extraction, Proceedings of the International Conference on Intelligent Robots and Systems 95, vol 1, pp. 249-256, 1995. 
[61] D. Panda, C.A. Anderson, M. Brenscheidt, P.G. Michalopoulos. Automatic Surveillance in Tunnels for Advanced Traffic Management Systems, Proceedings of the 1996 Annual Meeting of ITS AMERICA, pp. 412-421, April 1996.

[62] A. Polk, J.M. Kranig, P.E., E.D. Minge, P.E. Field Test of Non-Intrusive Traffic Detection Technologies Proceedings of the 1996 Annual Meeting of ITS AMERICA, pp. 456-467, April 1996.

[63] R. Reeves and K. Kubik, Compressed Domain Image Matching Using Symmetric Convolution, 1997 IEEE TENCON - Speech and Image Technologies for Computing and Telecommunications, pp. 543-546.

[64] S. G. Ritchie, B. Abdulhai, A.E. Parkany, J. Sheu, R.L. Cheu, S.I. Khan. A Comprehensive System for Incident Detection on Freeways and Arterials, Proceedings of the 1995 Annual Meeting of ITS America, pp. 617-622, March 1995.

[65] M. Rosenblum and B. Gothard, A High Fidelity Multi-Sensor Scene Understanding Sytem for Autonomous Navigation, Proceedings of the IEEE Intelligent Vehicle 2000 Symposium, p.637, Octember 2000.

[66] L. Saxton, Mobility 2000 and the Roots of IVHS, United States Department of Transportation - Federal Highway Administration, 1993.

[67] J. Semmens, Rethinking Transit "Dollars and Sense": Unearthing the True Cost of Public Transit, Policy Study No. 243 (Los Angeles: Reason Public Policy Institute, August 1998), Table 5, p. 13, August 1998. 
[68] S.K. Skinner, Intermodal Surface Transportation Efficiency Act of 1991Summary, U.S. Department of Transportation, 1991

[69] M. Sonka, V. Hlavac, and R. Boyle, Image Processing, Analysis and Machine Vision, Chapman and Hall Computing 1993.

[70] M.J. Sullivan, C.A. Richards, C.E. Smith, O. Masoud, N.P. Papanikolopoulos. Pedestrian Tracking from a Stationary Camera Using Active Deformable Models, IEEE Intelligent Vehicle Symposium, pp. 90-95, September 1995.

[71] C.J. Taylor, J. Malik, and J. Weber, A Real-Time Approach to Stereopsis and Lane Finding, IEEE Intelligent Vehicle Symposium, p. 207, 1996.

[72] USDOT, A Summary - Transportation Equity Act for the 21st Century: Moving Americans into the 21st Century, 1998.

[73] Ed. L. Saxton, Special Issue on Highway Electronic Systems, IEEE Transactions on Vehicular Technology, Vol. VT-19, January 1970.

[74] J. Weber, D. Koller, Q.-T. Loung, J. Malik, New Results in Stereo-based Automatic Vehicle Guidance, Proc. IEEE Intelligent Vehicles '95, Detroit, pp. 530535, September 1995.

[75] Z. Zhang, R. Deriche, O. Faugeras, Q-T. Luong, A Robust Technique for Matching Two Uncalibrated Images Through the Recovery of the Unknown Epipolar Geometery, Artificial Intelligence, Vol. 78, Issue 1-2, pp. 87-119, October 1995. 
Websites referenced:

[76] http://www.itsa.org/ITSNEWS.NSF/5939d2c38ae4cf 168525668a00628f94/ 61373e1ff6894ba18525695a004749f5?DpenDocument

[77] http://www.onstar.com

[78] http://www . autonews . com/news. cms?news Id $=4624$

[79] http://www.berkeley.edu/news/media/releases/2001/01/17_traff.html

[80] http://www.sys.uea.ac.uk/ /klm/research/general_stereo.html

[81] http://www.sic.rma.ac.be/ yvinec/Mines/rectification.html

[82] http://www.cis.upenn.edu/ "cgeyer/calib/

[83] http://www.webopedia.com/TERM/t/telematics.html

[84] http://www.trafficland.com/

[85] S. Segenchuk, An Overview of Color Quantization Techniques, http://www .cs.wpi.edu/"matt/courses/cs563/talks/color_quant/CQindex.html

[86] T. West, Adaptive Cruise Control: Meet Your New Co-Pilot, Popular Science, http://www .popsci.com/popsci/auto/0 12543,426811,00 html 